\title{
Harmonic Besov spaces on the ball
}

\author{
Seçil Gergün \\ Department of Mathematics, Dokuz Eylül University \\ 35160 Buca, Izmir, Turkey \\ secil.gergun@deu.edu.tr \\ H. Turgay Kaptanoğlu \\ Department of Mathematics, Bilkent University \\ 06800 Ankara, Turkey \\ kaptan@fen.bilkent.edu.tr \\ http://www.fen.bilkent.edu.tr/ kaptan/ \\ A. Ersin Üreyen \\ Department of Mathematics, Anadolu University \\ 26470 Eskişehir, Turkey \\ aeureyen@anadolu.edu.tr \\ Received 10 March 2015 \\ Accepted 8 June 2016 \\ Published 25 July 2016
}

\begin{abstract}
We initiate a detailed study of two-parameter Besov spaces on the unit ball of $\mathbb{R}^{n}$ consisting of harmonic functions whose sufficiently high-order radial derivatives lie in harmonic Bergman spaces. We compute the reproducing kernels of those Besov spaces that are Hilbert spaces. The kernels are weighted infinite sums of zonal harmonics and natural radial fractional derivatives of the Poisson kernel. Estimates of the growth of kernels lead to characterization of integral transformations on Lebesgue classes. The transformations allow us to conclude that the order of the radial derivative is not a characteristic of a Besov space as long as it is above a certain threshold. Using kernels, we define generalized Bergman projections and characterize those that are bounded from Lebesgue classes onto Besov spaces. The projections provide integral representations for the functions in these spaces and also lead to characterizations of the functions in the spaces using partial derivatives. Several other applications follow from the integral representations such as atomic decomposition, growth at the boundary and of Fourier coefficients, inclusions among them, duality and interpolation relations, and a solution to the Gleason problem.
\end{abstract}

Keywords: Spherical harmonic; zonal harmonic; Gegenbauer (ultraspherical) polynomial; Poisson kernel; reproducing kernel; radial fractional derivative; Möbius transformation; Bergman space; Besov space; Hardy space; Bergman projection; atomic decomposition; boundary growth; Fourier coefficient; duality; interpolation; Gleason problem.

Mathematics Subject Classification 2010: 31B05, 31B10, 31C25, 26A33, 33C55, 42B35, 45P05, 46E22, 46E15, 46E20, 47B34, 47B32, 47G10 


\section{Introduction}

Let $\mathbb{B}$ and $\mathbb{S}$ be the open unit ball and its boundary, the unit sphere in $\mathbb{R}^{n}$ with respect to the usual inner product $x \cdot y=x_{1} y_{1}+\cdots+x_{n} y_{n}$ and the norm $|x|=\sqrt{x \cdot x}$, where always $n \geq 2$. We write

$$
x=r \xi, \quad y=\rho \eta \quad \text { with } r=|x|, \rho=|y|, \text { and } \xi, \eta \in \mathbb{S},
$$

and use these throughout without further comment. When $n=2$, the ball is just the unit disk $\mathbb{D}$ in the complex plane bounded by the unit circle $\mathbb{T}$, and $x, y$ are complex numbers of modulus less than 1 .

We let $\nu$ and $\sigma$ be the volume and surface measures on $\mathbb{B}$ and $\mathbb{S}$ normalized as $\nu(\mathbb{B})=1$ and $\sigma(\mathbb{S})=1$. We take $q \in \mathbb{R}$ unrestricted unless explicitly said to the contrary and define on $\mathbb{B}$ the weighted volume measures

$$
d \nu_{q}(x)=\frac{1}{V_{q}}\left(1-|x|^{2}\right)^{q} d \nu(x)
$$

all of which are $\sigma$-finite. They are finite only for $q>-1$ and in such cases we choose the normalizing constants $V_{q}$ in order to have $\nu_{q}(\mathbb{B})=1$. So $V_{q}$ is a weighted normalized volume of $\mathbb{B}$ for $q>-1$. Naturally $V_{0}=1$. For $q \leq-1$, we set $V_{q}=1$.

We denote the Lebesgue classes with respect to $\nu_{q}$ by $L_{q}^{p}$. The Lebesgue class of essentially bounded functions on $\mathbb{B}$ with respect to any $\nu_{q}$ is the same; we denote it by $L^{\infty}$.

Harmonic functions by definition are those functions annihilated by the usual Laplacian $\Delta=\partial^{2} / \partial x_{1}^{2}+\cdots+\partial^{2} / \partial x_{n}^{2}$. We let $h(\mathbb{B})$ denote the space of complexvalued harmonic functions on $\mathbb{B}$ with the topology of uniform convergence on compact subsets. We denote by $h(\overline{\mathbb{B}})$ the space of harmonic functions on some $\varepsilon \mathbb{B}$ with $\varepsilon>1$. The space of bounded harmonic functions on $\mathbb{B}$ is denoted $h^{\infty}$.

The spaces under consideration in this paper form a two-parameter Sobolev-type family within $h(\mathbb{B})$ normed by a weighted integral of a suitable derivative, and we call them Besov spaces of harmonic functions. Harmonic Besov spaces have been studied early in [22-26] from a different perspective on more general domains.

The harmonic weighted Bergman spaces $b_{q}^{p}$ are the intersections $L_{q}^{p} \cap h(\mathbb{B})$ for $q>-1$ endowed with the norm of $L_{q}^{p}$. So a weighted Bergman space is imbedded isometrically in the Lebesgue class with the same parameters by inclusion. The subfamily $b_{q}^{2}$ consists of reproducing kernel Hilbert spaces with reproducing kernels $R_{q}$ with $q>-1$.

Our goal in this paper is to study in detail the harmonic Besov spaces which extend the Bergman spaces to all real $q$, and our notation for them is still $b_{q}^{p}$ with $q \in \mathbb{R}$. Our development rests on finding the reproducing kernels $R_{q}$ of the Hilbert Besov spaces with $q \leq-1$, which are not Bergman spaces.

The reproducing kernels give rise to radial fractional differential operators $D_{s}^{t}$ of order $t \in \mathbb{R}$ for any $s \in \mathbb{R}$ (so every $D_{s}^{0}=I$, the identity) that are specific to the spaces we want to define but still mapping $h(\mathbb{B})$ onto itself. These are discussed 
in detail in Sec. 3. We could use usual partial derivatives instead, and we have Theorem 1.2, but for the initial development, using our $D_{s}^{t}$ is more advantageous.

The holomorphic Besov spaces on $\mathbb{B}$ are studied in detail in $[2,17]$ among others. In this paper, we develop a theory for harmonic Besov spaces on $\mathbb{B}$ complementing those in these two references. Our job is more difficult because of two reasons. First, the reproducing kernel of the Bergman subfamily is a binomial with an explicit formula in the holomorphic case, and this makes the estimates on their growth much easier. In contrast, explicit usable formulas do not exist for the kernels in the harmonic case, and we have long sections on growth estimates to remove this deficiency. Second, one can resort to many earlier results on holomorphic spaces on $\mathbb{B}$, while the theory of harmonic spaces on $\mathbb{B}$ is still under development and we have to develop similar results within the confines of this paper.

The theory of Bergman spaces of harmonic functions $(q>-1)$ on various domains has been developed by many authors over the course of several years in numerous publications; see [4, 8, 16, 21, 29, 32], for example, and the references therein. Part of what we do in this single work is to complete the picture and extend the major results in the literature to Besov spaces of harmonic functions $(q \in \mathbb{R})$. Our main difficulty is the requirement to use derivatives to describe the functions in Besov spaces, and this necessitates the use of derivatives in every proof and every estimate, complicating every detail.

We define the harmonic Besov spaces $b_{q}^{p}$ by imbedding them isometrically in the Lebesgue classes with the same parameters $q, p$, much like it is done for the Bergman spaces, but the imbeddings are not inclusion for $q \leq-1$. Consider the linear transformations $I_{s}^{t}$ defined for $u \in h(\mathbb{B})$ by

$$
I_{s}^{t} u(x)=\left(1-|x|^{2}\right)^{t} D_{s}^{t} u(x) .
$$

Definition 1.1. For $q \in \mathbb{R}$ and $1 \leq p<\infty$, we set

$$
\begin{cases}s=0, \quad t=-q / p & \text { if } q \leq-1 \\ t=0 & \text { if } q>-1\end{cases}
$$

and define the harmonic Besov space $b_{q}^{p}$ to consist of all $u \in h(\mathbb{B})$ for which $I_{s}^{t} u$ belongs to $L_{q}^{p}$ endowed with the norm $\|u\|_{b_{q}^{p}}:=\left\|I_{s}^{t} u\right\|_{L_{q}^{p}}$.

So for $q>-1$, we identify Besov and Bergman spaces, and for $q \leq-1$, we map the Besov spaces to unweighted Bergman spaces by $D_{0}^{-q / p}$. We always take $1 \leq p<\infty$ in this paper and deliberately avoid a study of the case $p=\infty$ although many of our results naturally cover that case too.

It turns out that we obtain the same $b_{q}^{p}$ if use any $I_{s}^{t}$ with a sufficiently high $t$ for imbedding it in $L_{q}^{p}$ :

Theorem 1.1. For any $q \in \mathbb{R}, a u \in h(\mathbb{B})$ belongs to $b_{q}^{p}$ if and only if $I_{s}^{t} u$ belongs to $L_{q}^{p}$ for some (and therefore any) $s, t$ satisfying

$$
q+p t>-1 \text {. }
$$

The $L_{q}^{p}$ norm of $I_{s}^{t} u$ is equivalent to $\|u\|_{b_{q}^{p}}$ given in Definition 1.1. 
More precisely, this theorem says that $u$ belongs to $b_{q}^{p}$ if and only if $u$ is harmonic on $\mathbb{B}$ and

$$
\frac{1}{V_{q}} \int_{\mathbb{B}}\left|D_{s}^{t} u(x)\right|^{p}\left(1-|x|^{2}\right)^{q+p t} d \nu(x)<\infty
$$

for some $s, t$ satisfying (1.2). There is no restriction on $s$ in (1.2). So the initial choice of $t$ in (1.1) for $q \leq-1$ is for the simplicity of not having any $1-|x|^{2}$ in the integral. Note that $t$ can take only positive values for $q \leq-1$, but it can take negative values as well for $q>-1$. This way we also have a lot of other equivalent norms also on harmonic Bergman spaces, some involving indefinite integrals of fractional order of the functions.

Until Sec. 9 where we prove Theorem 1.1, whenever we mention Besov spaces, we use the $s, t$ given in (1.1), with or without mention.

We go beyond $D_{s}^{t}$ by showing that they can be replaced by the usual radial derivatives $\mathcal{R}^{l}$ or partial derivatives $\partial^{\alpha}$, whose precise definitions are in Sec. 2 .

Theorem 1.2. For $q \in \mathbb{R}$ and $u \in h(\mathbb{B})$, the following are equivalent:

(a) $u \in b_{q}^{p}$.

(b) For every $l \in \mathbb{N}$ with $q+p l>-1$ and for every multi-index $\alpha$ with $|\alpha|=l$, we have $\left(1-|x|^{2}\right)^{l}\left(\partial^{\alpha} u\right)(x) \in L_{q}^{p}$, that is, $\partial^{\alpha} u \in b_{q+p l}^{p}$.

(c) There exists an $l \in \mathbb{N}$ with $q+p l>-1$ such that for every multi-index $\alpha$ with $|\alpha|=l$, we have $\left(1-|x|^{2}\right)^{l}\left(\partial^{\alpha} u\right)(x) \in L_{q}^{p}$, that is, $\partial^{\alpha} u \in b_{q+p l}^{p}$.

(d) For every $l \in \mathbb{N}$ with $q+p l>-1$, we have $\left(1-|x|^{2}\right)^{l}\left(\mathcal{R}^{l} u\right)(x) \in L_{q}^{p}$, that is, $\mathcal{R}^{l} u \in b_{q+p l}^{p}$.

(e) There exists an $l \in \mathbb{N}$ with $q+p l>-1$ such that $\left(1-|x|^{2}\right)^{l}\left(\mathcal{R}^{l} u\right)(x) \in L_{q}^{p}$, that $i s, \mathcal{R}^{l} u \in b_{q+p l}^{p}$.

Definition 1.1 assigns the space $b_{q}^{p}$ to the point $(p, q)$ in the half plane $\{\operatorname{Re} p \geq 1\}$. Our main interest lies in the lower half $q \leq-1$ of this region (proper Besov zone), but our results cover and generalize what is known for the upper half $q>-1$ (weighted Bergman zone) as well.

The starting point of all, including the differential operators $D_{s}^{t}$, is the reproducing kernels of $b_{q}^{2}$. So we identify them first, which are new for $q<-1$, although the proof of the reproducing property comes later.

Theorem 1.3. The spaces $b_{q}^{2}$ are reproducing kernel Hilbert spaces and their reproducing kernels have the form

$$
R_{q}(x, y)=\sum_{m=0}^{\infty} \gamma_{m}(q) Z_{m}(x, y) \quad(q \in \mathbb{R}, x, y \in \mathbb{B}),
$$

where the $\gamma_{m}(q)$ are given in Definition 3.1, and the $Z_{m}(x, y)$ are the zonal harmonics explained in Sec. 14.

A good part of this paper is geared toward finding all bounded harmonic Bergman-Besov projections from the $L_{q}^{p}$ onto the $b_{q}^{p}$. 
Definition 1.2. For $s \in \mathbb{R}$, the harmonic Bergman-Besov projections are the linear transformations defined by

$$
Q_{s} f(x)=\int_{\mathbb{B}} f(y) R_{s}(x, y) d \nu_{s}(y)
$$

for suitable $f$.

For $s>-1$, the $Q_{s}$ are just the usual harmonic Bergman projections.

Theorem 1.4. For $q, s \in \mathbb{R}$ and $1 \leq p<\infty, Q_{s}: L_{q}^{p} \rightarrow b_{q}^{p}$ is bounded if and only if

$$
q+1<p(s+1)
$$

Given an s satisfying (1.3), if $t$ satisfies (1.2), then for $u \in b_{q}^{p}$,

$$
Q_{s} I_{s}^{t} u=\frac{V_{s+t}}{V_{s}} u
$$

Thus $Q_{s}: L_{q}^{p} \rightarrow b_{q}^{p}$ is surjective and $I_{s}^{t}: b_{q}^{p} \rightarrow L_{q}^{p}$ is an imbedding. Note that (1.2) and (1.3) together imply $s+t>-1$. For such $s, t$, each of $\left(V_{s} / V_{s+t}\right) I_{s}^{t}$ is a right inverse for $Q_{s}$ on $L_{q}^{p}$, and $\left(V_{s} / V_{s+t}\right) Q_{s}$ is a left inverse for $I_{s}^{t}$ on $b_{q}^{p}$. Moreover, (1.4) is a family of integral representations for $u \in b_{q}^{p}$ which take the form

$$
u(x)=\frac{1}{V_{s+t}} \int_{\mathbb{B}} D_{s}^{t} u(y) R_{s}(x, y)\left(1-|y|^{2}\right)^{s+t} d \nu(y) \quad(x \in \mathbb{B})
$$

when written explicitly.

The inequality (1.3) that characterizes the boundedness of Bergman-Besov projections is the same even for holomorphic Bergman-Besov projections on the ball (see [17, Theorem 1.2]) and harmonic Bergman projections on the upper half space (see [21, Theorem 4.3]). However we do not attempt to make any comparisons with similar results in the literature on holomorphic spaces or spaces of other types of harmonic functions (pluriharmonic, $\mathcal{M}$-harmonic, etc.) or harmonic functions on other domains such as the upper half space.

The proof of Theorem 1.4 depends on two basic tools. The first are certain estimates on weighted integrals of powers of $R_{q}(x, y)$ that are of interest in their own right.

Theorem 1.5. For $q \in \mathbb{R}, a>0$, and $b>-1$, set $c=a(n+q)-(n+b)$. Then

$$
\mathbb{I}_{q, a, b}(x)=\int_{\mathbb{B}}\left|R_{q}(x, y)\right|^{a}\left(1-|y|^{2}\right)^{b} d \nu(y) \sim \begin{cases}1 & \text { if } c<0 \\ \frac{1}{|x|^{2}} \log \frac{1}{1-|x|^{2}} & \text { if } c=0 \\ \frac{1}{\left(1-|x|^{2}\right)^{c}} & \text { if } c>0 ;\end{cases}
$$

for $x \in \mathbb{B}$, where the meaning of $\sim$ is explained in Sec. 2. Moreover, when $c<0$, $\mathbb{I}_{q, a, b}(x)$ extends continuously to all $x \in \overline{\mathbb{B}}$. 
The other are certain integral transforms on weighted Lebesgue spaces and the conditions on their boundedness. Our operators are the following for $s, t \in \mathbb{R}$ and we let them act on $L_{q}^{p}$ :

$$
\begin{aligned}
& T_{s, t} f(x)=\left(1-|x|^{2}\right)^{t} \int_{\mathbb{B}} f(y) R_{s+t}(x, y)\left(1-|y|^{2}\right)^{s} d \nu(y), \\
& S_{s, t} f(x)=\left(1-|x|^{2}\right)^{t} \int_{\mathbb{B}} f(y)\left|R_{s+t}(x, y)\right|\left(1-|y|^{2}\right)^{s} d \nu(y), \\
& E_{s, t} f(x)=\left(1-|x|^{2}\right)^{t} \int_{\mathbb{B}} f(y) \frac{1}{\{x, y\}^{(n+s+t) / 2}}\left(1-|y|^{2}\right)^{s} d \nu(y), \\
& X_{s, t} f(x)=\int_{\mathbb{B}} f(y) K_{s, t}(x, y) d \nu(y),
\end{aligned}
$$

where $K_{s, t}(x, y)$ is a measurable kernel satisfying

$$
\left|K_{s, t}(x, y)\right| \lesssim \frac{\left(1-|x|^{2}\right)^{t}\left(1-|y|^{2}\right)^{s}}{\{x, y\}^{(n+s+t) / 2}} \quad(x, y \in \mathbb{B}),
$$

and $\{x, y\}$ is defined in (2.3).

Theorem 1.6. The conditions (a)-(d) are equivalent, and they imply (e):

(a) $T_{s, t}$ is bounded on $L_{q}^{p}$.

(b) $S_{s, t}$ is bounded on $L_{q}^{p}$.

(c) $-p t<q+1<p(s+1)$.

(d) $E_{s, t}$ is bounded on $L_{q}^{p}$.

(e) $X_{s, t}$ is bounded on $L_{q}^{p}$.

Note that the conditions (1.2) and (1.3) are derived from Theorem 1.6.

The paper is organized as follows.

Section 2 is for our rather standard notation and some other well-known formulas. We place some standard known material in Sec. 14, where we review spherical harmonics on which everything else is based as well as the Bergman kernels that are already known. In Sec. 3, we define the Besov kernels and the radial differential operators that are used in defining the harmonic Besov spaces. We give basic properties of Besov spaces in Sec. 4.

We concentrate on the Hilbert Besov spaces in Sec. 5, and show that they and the Lebesgue classes $L_{q}^{2}$ can be decomposed in terms of spherical harmonics. Naturally the proof of Theorem 1.3 is also here. Section 6 is for calculating some integrals used mainly in the estimation of Theorem 1.5, where we also prove a different Schur test for the $L^{p}$-boundedness of the integral transforms of Theorem 1.6. In Sec. 7, we give several estimates on the growth of the Besov kernels and also prove Theorem 1.5. In Sec. 8, we prove Theorem 1.6. In Sec. 9, we prove Theorem 1.1. Section 10 is for real Möbius transformations, the hyperbolic metric, and an application of Theorem 1.1 to atomic decompositions in $b_{q}^{p}$ spaces. 
Section 11 is devoted to the proof of Theorem 1.4. The proof of Theorem 1.2 occupies Sec. 12 and it is an important application of Theorem 1.4. Various other applications of Bergman projections are collected in Sec. 13. One of these is on the growth of generalized Fourier coefficients and another on the boundary growth of functions in Besov spaces. They also yield refined inclusions of the $b_{q}^{p}$ in other $b_{q}^{p}$ with different parameters. Other applications here are to determine duals of and complex interpolation among the $b_{q}^{p}$ spaces, and to a solution of the Gleason problem in them.

A few of the results in this paper have been announced in [13], and a comparison of the results in [13] with the results on holomorphic spaces has been made in [18].

\section{Notation and Preliminaries}

In multi-index notation, $\alpha=\left(\alpha_{1}, \ldots, \alpha_{n}\right) \in \mathbb{N}^{n}$ is an $n$-tuple of nonnegative integers, $|\alpha|=\alpha_{1}+\cdots+\alpha_{n}, \alpha !=\alpha_{1} ! \cdots \alpha_{n} !, 0^{0}=1, x^{\alpha}=x_{1}^{\alpha_{1}} \cdots x_{n}^{\alpha_{n}}$, and

$$
\partial^{\alpha}=\frac{\partial^{|\alpha|}}{\partial x^{\alpha}} .
$$

We also use $\partial_{i}=\partial / \partial x_{i}$, which is not about multi-indices.

An overline $\overline{(\cdot)}$ denotes closure for sets and complex conjugation for elements. The exponent conjugate to $p$ is $p^{\prime}=p /(p-1)$. If $f$ is a function defined on $\mathbb{B}$, its dilates are the functions $f_{\tau}$ defined when $\tau>0$ by $f_{\tau}(x)=f(\tau x)$ for $x \in \frac{1}{\tau} \mathbb{B}$.

The Pochhammer symbol $(a)_{b}$ is defined by

$$
(a)_{b}=\frac{\Gamma(a+b)}{\Gamma(a)}
$$

when $a$ and $a+b$ are off the pole set $-\mathbb{N}$ of the gamma function $\Gamma$. This is a shifted rising factorial since $(a)_{k}=a(a+1) \cdots(a+k-1)$ for positive integer $k$. In particular, $(1)_{k}=k$ ! and $(a)_{0}=1$. Stirling formula gives

$$
\frac{\Gamma(c+a)}{\Gamma(c+b)} \sim c^{a-b}, \quad \frac{(a)_{c}}{(b)_{c}} \sim c^{a-b}, \quad \frac{(c)_{a}}{(c)_{b}} \sim c^{a-b} \quad(\operatorname{Re} c \rightarrow \infty),
$$

where $A \sim B$ means that $|A / B|$ is bounded above and below by two positive constants, that is, $A=\mathcal{O}(B)$ and $B=\mathcal{O}(A)$, for all $A, B$ of interest. So for example, $1-|x| \sim 1-|x|^{2}$ for all $x \in \mathbb{B}$. Such constants that are independent of the parameters and the functions in the equation are all denoted by the generic unadorned upper case $C$. We also use $A \lesssim B$ to mean $A=\mathcal{O}(B)$.

The beta integral in two forms and its value are

$$
2 \int_{0}^{1} r^{2 a-1}\left(1-r^{2}\right)^{b-1} d r=\int_{0}^{1} r^{a-1}(1-r)^{b-1} d r=B(a, b)=\frac{\Gamma(a) \Gamma(b)}{\Gamma(a+b)}
$$

for $a, b>0$. The polar coordinates formula is

$$
\int_{\mathbb{B}} f(x) d \nu(x)=n \int_{0}^{1} r^{n-1} \int_{\mathbb{S}} f(r \xi) d \sigma(\xi) d r \quad\left(f \in L_{0}^{1}\right) .
$$


We find out from this formula that $\nu(\varepsilon \mathbb{B})=\varepsilon^{n}$. Many of our integrals over $\mathbb{B}$ are improper at $\mathbb{S}$, and they should be considered as the limit as $\varepsilon \rightarrow 1^{-}$of the integral over $\varepsilon \mathbb{B}$, and this is the same as considering the polar coordinates formula whose integral in the radial direction is over $[0, \varepsilon]$ followed by the limit as $\varepsilon \rightarrow 1^{-}$.

The exact value of the normalizing coefficient $V_{q}$ of $\nu_{q}$ for $q>-1$ can be computed by applying the polar coordinates formula to $f(x)=\left(1-|x|^{2}\right)^{q}$ and using the beta integral. It turns out that

$$
V_{q}=\frac{(1)_{n / 2}}{(1+q)_{n / 2}} \quad(q>-1) \text {. }
$$

Thus $V_{q} \sim 1 / n^{q}$ for $n$ large. Also $V_{q}=1 /(1+q)$ when $n=2$.

The normalized measures $\nu_{q}$ converge weak* to $\sigma$ as $q \rightarrow-1^{+}$. This means that if $f$ is continuous on $\overline{\mathbb{B}}$, then

$$
\lim _{q \rightarrow-1^{+}} \int_{\mathbb{B}} f d \nu_{q}=\int_{\mathbb{S}} f d \sigma
$$

For a proof, see [19, pp. 173-174].

For convenience, we use the abbreviation

$$
\{x, y\}=1-2 x \cdot y+|x|^{2}|y|^{2}
$$

for which $\{x, x\}=\left(1-|x|^{2}\right)^{2}$. This is the real-variable counterpart of the complex quantity $|1-z \bar{w}|^{2}$. Note that $0<(1-r \rho)^{2} \leq\{x, y\} \leq(1+r \rho)^{2}<4$ for $x, y \in \mathbb{B}$. Further,

$\{x, y\}=\{\rho x, \eta\} \quad$ and $\quad\{x, \eta\}=1-2 r \xi \cdot \eta+r^{2}=(\eta-x) \cdot(\eta-x)=|x-\eta|^{2}$,

hence the quantity $\{\xi, \eta\}^{1 / 2}$ is the Euclidean metric restricted to $\mathbb{S}$. The Poisson kernel for $\mathbb{B}$ is

$$
P(x, \eta)=\frac{1-|x|^{2}}{|x-\eta|^{n}}=\frac{1-r^{2}}{\{x, \eta\}^{n / 2}} \quad(x \in \mathbb{B}, \eta \in \mathbb{S}) .
$$

The usual radial derivative $\mathcal{R}$ of a differentiable function $f$ is given by

$$
\mathcal{R} f(x)=x \cdot \nabla f(x)=\frac{\partial}{\partial \tau}(f(\tau x))_{\tau=1}=\sum_{m=1}^{\infty} m f_{m}(x),
$$

in which $\nabla$ denotes the usual gradient, and the last form is valid for a real-analytic $f$ with homogeneous expansion $f=\sum_{m=0}^{\infty} f_{m}$. The fundamental theorem of calculus shows that

$$
f(x)-f(0)=\int_{0}^{1}(\mathcal{R} f)(\tau x) \frac{d \tau}{\tau} .
$$

On a few occasions we make use of the binomial expansion

$$
\frac{1}{(1-z)^{a}}=\sum_{m=0}^{\infty} \frac{(a)_{m}}{m !} z^{m} \quad(a \notin-\mathbb{N}, z \in \mathbb{D})
$$


whose coefficients satisfy $\sim m^{a-1}$. The classical hypergeometric function is

$$
{ }_{2} F_{1}(a, b ; c ; z)=\sum_{m=0}^{\infty} \frac{(a)_{m}(b)_{m}}{(c)_{m}} \frac{z^{m}}{m !} \quad(z \in \mathbb{D}),
$$

where $a, b \in \mathbb{R}$ and $c>0$. It converges absolutely for $z \in \mathbb{D}$ and uniformly on its compact subsets. The Chu-Vandermonde identity is

$$
{ }_{2} F_{1}(-m, b ; c ; 1)=\frac{(c-b)_{m}}{(c)_{m}} \quad(m \in \mathbb{N}, c \notin-\mathbb{N}) ;
$$

see [30, Formula 15.4.24].

We denote an integral inner product on a function space $H$ by $[\cdot, \cdot]_{H}$ and the associated norm by $\|\cdot\|_{H}$.

Definition 2.1. A function $K(x, y)$ is called a reproducing kernel for a Hilbert space $H$ of functions defined on $\mathbb{B}$ if $K(x, \cdot) \in H$ for each $x \in \mathbb{B}$ and

$$
u(x)=[u(\cdot), K(x, \cdot)]_{H} \quad(u \in H, x \in \mathbb{B}) .
$$

The kernel $K$ of such an $H$ is unique; and a given positive definite $K$ determines a unique $H$.

\section{Harmonic Besov Kernels and Radial Differential Operators}

We start by recalling from Sec. 14 the reproducing kernels

$$
R_{q}(x, y)=\sum_{m=0}^{\infty} \gamma_{m}(q) Z_{m}(x, y)=\sum_{m=0}^{\infty} \frac{(1+n / 2+q)_{m}}{(n / 2)_{m}} Z_{m}(x, y)
$$

of weighted harmonic Bergman spaces $b_{q}^{2}$ on $\mathbb{B}$, for which $q>-1$.

However, we notice that the coefficients $\gamma_{m}(q)$ in (3.1) make sense as long as $q>-(1+n / 2)$, and for all such $q$, they satisfy

$$
\gamma_{m}(q) \sim m^{1+q} \quad(m \rightarrow \infty)
$$

by (2.1). The infinite sums in (3.1) considered for $-(1+n / 2)<q \leq-1$ have at least the same convergence properties on $\mathbb{B} \times \mathbb{B}$ as those of Bergman kernels with which $q>-1$. Since $\gamma_{m}(q)>0$ for all $m$ and $q>-(1+n / 2)$, and the $Z_{m}$ are positive definite kernels, by convergence we conclude that $R_{q}$ given as in (3.1) is a positive definite function, and thus is a reproducing kernel and generates a reproducing kernel Hilbert space $b_{q}^{2}$ on $\mathbb{B}$ for all $q>-(1+n / 2)$.

We now extend the kernels to all real $q$ and this allows us to define the radial differential operators $D_{s}^{t}$ acting on $h(\mathbb{B})$. The two are related, because both the kernels and the $D_{s}^{t}$ act as coefficient multipliers on the homogeneous expansions of the members of their respective domains.

In the holomorphic category which we take as a model, the Bergman kernels are generalized to Besov kernels in [2, p. 13] by switching to hypergeometric functions from binomials. From the particular hypergeometric functions picked, it is clear 
that the essential property that is preserved is the growth rate of the coefficients of the homogeneous series expansion of the Bergman kernels in terms of the powers of the Hermitian inner product of $z$ and $w$ in $\mathbb{B} \subset \mathbb{C}^{N}$. With harmonic Bergman kernels, we also have homogeneous expansions (3.1) in which the zonal harmonics replace the powers of the inner product, and the growth rate (3.2) of the coefficients is uniform for $q>-1$. So the main idea here is to replace the coefficients $\gamma_{m}(q)$ of $Z_{m}$ in $R_{q}$ by other $\gamma_{m}(q)$ that preserve the growth rate of (3.2) for $q \leq-(1+n / 2)$ as well.

Definition 3.1. For $m=0,1,2, \ldots$, we set

$$
\gamma_{m}(q):= \begin{cases}\frac{(1+n / 2+q)_{m}}{(n / 2)_{m}} & \text { if } q>-(1+n / 2) ; \\ \frac{(m !)^{2}}{(1-(n / 2+q))_{m}(n / 2)_{m}} & \text { if } q \leq-(1+n / 2) ;\end{cases}
$$

and define

$$
R_{q}(x, y):=\sum_{m=0}^{\infty} \gamma_{m}(q) Z_{m}(x, y)=\sum_{m=0}^{\infty} \gamma_{m}(q) \sum_{k=1}^{\delta_{m}} Y_{m k}(x) \overline{Y_{m k}(y)}
$$

wherever the series converges.

The kernels (3.3) for $q \leq-(1+n / 2)$ are new. They have appeared first in our research announcement [13].

By the definition of the Pochhammer symbol, $\gamma_{m}(q)>0$ for all $m=0,1,2, \ldots$ and all $q \in \mathbb{R}$. In particular, $\gamma_{0}(q)=1$ for all $q$, and thus

$$
R_{q}(0, y)=R_{q}(x, 0)=1 \quad(q \in \mathbb{R}) .
$$

Also clearly $R_{q}(x, y)=R_{q}(y, x)$ and $R_{q}(x, x)>0$ for all $q$ since the same is true for all $Z_{m}$ by (14.3). A byproduct of the connections (14.8) and those following it is that the $R_{q}$ depend on $x$ and $y$ via $x \cdot y$.

By (2.1),

$$
\gamma_{m}(q) \sim m^{1+q} \quad(m \rightarrow \infty)
$$

now also for $q \leq-1$ as well as $q>-1$ as promised. Although it is possible to write the series defining the $R_{q}$ as multiple hypergeometric functions [20], we cannot make use of these complicated expressions. Further, by (14.1),

$$
\begin{aligned}
\left|R_{q}(x, y)\right| & \leq \sum_{m=0}^{\infty} \gamma_{m}(q) r^{m} \rho^{m}\left|Z_{m}(\xi, \eta)\right| \\
& \lesssim 1+\sum_{m=1}^{\infty} m^{1+q}(r \rho)^{m} \delta_{m} \sim 1+\sum_{m=1}^{\infty} m^{n-1+q}(r \rho)^{m} .
\end{aligned}
$$

The geometric factor $\left((r \rho)^{m}\right)$ dominates the polynomial factor $\left(m^{n-1+q}\right)$ eventually if $r \rho<1$ and this suffices for convergence. 
Proposition 3.1. The series (3.3) converges absolutely and uniformly on subsets of $\mathbb{R}^{n} \times \mathbb{R}^{n}$ whose elements $(x, y)$ satisfy $r \rho \leq \varepsilon<1$, in particular, on $K \times \mathbb{S}$ for $K \subset \mathbb{B}$ compact. Consequently, $R_{q}$ is harmonic as a function of either of its variables on $\mathbb{B}$. Moreover, for $q<-n, R_{q}$ converges absolutely and uniformly and thus is continuous on $\overline{\mathbb{B}} \times \overline{\mathbb{B}}$.

The calculation in (3.6) yields a little more with the help of the binomial expansion and other power series.

Proposition 3.2. For $x \in \mathbb{B}$ and $y \in \overline{\mathbb{B}}$,

$$
\left|R_{q}(x, y)\right| \lesssim \begin{cases}1 & \text { if } q<-n \\ (|x||y|)^{-1} \log (1-|x||y|)^{-1} & \text { if } q=-n \\ (1-|x||y|)^{-(n+q)}, & \text { if } q>-n .\end{cases}
$$

If additionally $y=\lambda x$ with $\lambda>0$, then $R_{q}(x, y)>0$ and we have also $\gtrsim$.

Proposition 3.2 extends various such estimates for weighted Bergman kernels (see [29, Proposition 4], for example) to all $q \in \mathbb{R}$. In Sec. 7, we have finer estimates with both $\lesssim$ and $\gtrsim$.

There are three critical values of $q$ : $-1,-(1+n / 2)$, and $-n$. At $q=-1$, we pass between Bergman spaces and proper Besov spaces. At $q=-(1+n / 2)$, we pass between Bergman-type kernels and hypergeometric kernels. At $q=-n$, we pass between unbounded kernels and bounded kernels. When $n=2$, the latter two critical values are the same. For comparison, for holomorphic kernels, there are only two critical values, because the latter two critical values are merged.

Let us indicate the precise relationship of the new kernels to the holomorphic kernels when $n=2$ and thus $q \leq-2$.

$$
\begin{aligned}
R_{q}(x, y) & =1+\sum_{m=1}^{\infty} \frac{(1)_{m}(1)_{m}}{(-q)_{m} m !}\left(x^{m} \bar{y}^{m}+\bar{x}^{m} y^{m}\right) \\
& ={ }_{2} F_{1}(1,1 ;-q ; x \bar{y})+{ }_{2} F_{1}(1,1 ;-q ; \bar{x} y)-1=2 \operatorname{Re} K_{q}(x, y)-1 .
\end{aligned}
$$

The kernels given in $[2$, p. 13$]$ differ by the constant multiple $(-1-q)^{-1}$ from the hypergeometric functions above, but the $K_{q}$ here and their kernels generate the same spaces. In particular, for $q=-2$,

$$
R_{-2}(x, y)=1+\sum_{m=1}^{\infty} \frac{1}{1+m}\left(x^{m} \bar{y}^{m}+\bar{x}^{m} y^{m}\right)=2 \operatorname{Re}\left(\frac{1}{x \bar{y}} \log \frac{1}{1-x \bar{y}}\right)-1 .
$$

We name the space $b_{-n}^{2}$ the harmonic Dirichlet space, because its kernel $R_{-n}$ has logarithmic behavior, and when $n=2, R_{-2}$ is directly related to the logarithmic kernel $K_{-2}$ of the holomorphic Dirichlet space.

One property of the harmonic Bergman kernels in $[1,29]$ is that for nonnegative integer $q, R_{q}$ can be written as certain derivatives of order $1+q$ of the Poisson kernel. We want to extend this relationship to the new kernels defined for $q \leq-(1+n / 2)$, 
to noninteger orders, and also simplify it using radial differential operators in a manner exemplified in the holomorphic setting in [17, Eq. (13)]. Radial differential operators act as coefficient multipliers on the homogeneous expansions of functions and yield functions of the same kind even for real orders of differentiation. The idea is to pick the coefficients in such a way that derivatives of the correct order are obtained and the said relations are fulfilled among the kernels.

Definition 3.2. Let $u=\sum_{m=0}^{\infty} u_{m} \in h(\mathbb{B})$ be given by its homogeneous expansion (14.9). We define radial differential operators $D_{s}^{t}$ of order $t$ by

$$
D_{s}^{t} u:=\sum_{m=0}^{\infty} d_{m}(s, t) u_{m}:=\sum_{m=0}^{\infty} \frac{\gamma_{m}(s+t)}{\gamma_{m}(s)} u_{m} .
$$

Note that $d_{0}(s, t)=1$ for all $s, t$. Explicitly,

$$
d_{m}(s, t)= \begin{cases}\frac{(1+n / 2+s+t)_{m}}{(1+n / 2+s)_{m}} & \text { if } s>-(1+n / 2), s+t>-(1+n / 2) ; \\ \frac{(1+n / 2+s+t)_{m}(1-n / 2-s)_{m}}{(m !)^{2}} & \text { if } s \leq-(1+n / 2), s+t>-(1+n / 2) ; \\ \frac{(m !)^{2}}{(1+n / 2+s)_{m}(1-n / 2-s-t)_{m}} & \text { if } s>-(1+n / 2), s+t \leq-(1+n / 2) ; \\ \frac{(1-n / 2-s)_{m}}{(1-n / 2-s-t)_{m}} & \text { if } s \leq-(1+n / 2), s+t \leq-(1+n / 2) .\end{cases}
$$

This is not the only possible way to choose the $d_{m}(s, t)$. Other positive numbers would do as long as (3.8) is satisfied. Note that $s$ appears in $d_{m}(s, t)$ twice in such a way that its effect on growth is canceled, and might at first seem redundant. Our particular choice is for having nice exact formulas like (3.10) or (3.11). Without them, the proofs would be more cumbersome.

We list some immediate properties of $D_{s}^{t}: d_{m}(s, 0)=1$ for all $m, s$, so $D_{s}^{0}=I$, the identity; $D_{s}^{t} 1=1$; and $d_{m}(s, t) \neq 0$ for all choices of $m, s, t$. Further, if $u$ belongs to the space $\mathcal{H}_{m}$ of harmonic homogeneous polynomials of degree $m$ as explained early in Sec. 14, then $D_{s}^{t} u=d_{m}(s, t) u$. Thus in every case

$$
D_{s}^{t}\left(\mathcal{H}_{m}\right)=\mathcal{H}_{m}
$$

Further, by Definition 3.1 and (2.1),

$$
d_{m}(s, t) \sim m^{t} \quad(m \rightarrow \infty) .
$$

Particularly, $D_{-n / 2}^{1}=\mathcal{R}+I$. The last two properties justify the term radial differential operator of order $t$ for $D_{s}^{t}$. The fact that its coefficients are all nonzero causes every $D_{s}^{t}$ to be invertible with two-sided inverse

$$
\left(D_{s}^{t}\right)^{-1}=D_{s+t}^{-t}
$$


which follows from the additive property

$$
D_{s+t_{1}}^{t_{2}} D_{s}^{t_{1}}=D_{s}^{t_{1}+t_{2}}
$$

The operators $D_{s}^{t}$ are constructed so that in all cases

$$
D_{s}^{t} R_{s}(x, y)=R_{s+t}(x, y),
$$

where differentiation is performed only on one of the variables $x, y$; and by symmetry it does not matter which. In particular,

$$
R_{q}(x, y)=D_{-1}^{1+q} P(x, y) \quad(q \in \mathbb{R})
$$

extending [12, Eq. (3.1); 1, Eq. (8.12); 14, Lemma 2.5], a formula in [29, p. 29], and [33, Lemma $3.1 ; 4$, Lemma 2], all of which handle at most $q>-1$.

Perhaps the greatest advantage of the $D_{s}^{t}$ is that they map $h(\mathbb{B})$ to itself.

Theorem 3.1. If $u \in h(\mathbb{B})$, then every $D_{s}^{t} u=\sum_{m=0}^{\infty} d_{m}(s, t) u_{m}$ converges absolutely and uniformly on compact subsets of $\mathbb{B}$ and thus is harmonic there. Moreover, $D_{s}^{t}$ maps $h(\mathbb{B})$ onto itself.

Proof. Let $K$ be a compact subset of $\mathbb{B}$, and choose $\tau<1$ so that $K \subset \tau \mathbb{B}$. For $x=r \xi \in K$, it holds that $r / \tau<1$ and

$$
\left|\sum_{m=0}^{\infty} d_{m}(s, t) u_{m}(x)\right| \lesssim \sum_{m=0}^{\infty} m^{t}\left(\frac{r}{\tau}\right)^{m}\left|u_{m}(\tau \xi)\right| \lesssim \sum_{m=0}^{\infty}\left|u_{m}(\tau \xi)\right|
$$

by (3.8) and homogeneity. The convergence of the right-hand side uniformly on the compact subset $\tau \mathbb{S}$ implies the absolute and uniform convergence of the left-hand side for $x \in K$. The surjectivity of $D_{s}^{t}$ follows from the invertibility of $D_{s}^{t}$.

A special case of this is mentioned in [1, Exercises 1.12 and 5.23].

Remark 3.1. Combining Proposition 3.1 with Theorem 3.1, we conclude that $D_{s}^{t} R_{q}$ is harmonic in $x, y \in \mathbb{B}$ for any values of $q, s, t$ and uniformly bounded on $K \times \mathbb{S}$ for $K \subset \mathbb{B}$ compact.

Theorem 3.2. Suppose $\left\{u^{j}\right\}$ is a sequence of harmonic functions on $\mathbb{B}$ converging uniformly on compact subsets of $\mathbb{B}$ to $u \in h(\mathbb{B})$. Then $\left\{D_{s}^{t} u^{j}\right\}$ converges to $D_{s}^{t} u$ uniformly on compact subsets of $\mathbb{B}$. In other words, the action of $D_{s}^{t}$ on $h(\mathbb{B})$ is continuous.

Proof. Let $K \subset \mathbb{B}$ be compact and choose $\tau<1$ such that $K \subset \tau \mathbb{B}$. Then for $x=r \xi \in K$, we have $r / \tau<1$. By using Definition 3.2, (14.10) which is the crucial step, Theorem 3.1, (3.8), homogeneity, (14.7), (14.1), for all $x \in K$, we obtain

$$
\begin{aligned}
\left|D_{s}^{t} u^{j}(x)-D_{s}^{t} u(x)\right| & =\left|\sum_{m=0}^{\infty} d_{m}(s, t)\left(u_{m}^{j}(x)-u_{m}(x)\right)\right| \\
& =\left|\sum_{m=0}^{\infty} d_{m}(s, t) \frac{1}{\tau^{m}} \int_{\mathbb{S}}\left(u^{j}(\tau \eta)-u(\tau \eta)\right) r^{m} Z_{m}(\xi, \eta) d \sigma(\eta)\right|
\end{aligned}
$$




$$
\begin{aligned}
& \leq \int_{\mathbb{S}}\left|u^{j}(\tau \eta)-u(\tau \eta)\right| \sum_{m=0}^{\infty}\left(\frac{r}{\tau}\right)^{m} d_{m}(s, t) \delta_{m} d \sigma(\eta) \\
& \lesssim \int_{\mathbb{S}}\left|u^{j}(\tau \eta)-u(\tau \eta)\right|\left(1+\sum_{m=1}^{\infty}\left(\frac{r}{\tau}\right)^{m} m^{n-2+t}\right) d \sigma(\eta) \\
& \lesssim \int_{\mathbb{S}}\left|u^{j}(\tau \eta)-u(\tau \eta)\right| d \sigma(\eta) .
\end{aligned}
$$

Now the uniform convergence of $\left\{u^{j}\right\}$ on the compact set $\tau \mathbb{S}$ implies the uniform convergence of $\left\{D_{s}^{t} u^{j}\right\}$ on $K$.

\section{Harmonic Besov Spaces}

Now all the technical details required for Definition 1.1 are established and we have the two-parameter Besov space family $b_{q}^{p}$ at hand.

Harmonic Besov spaces appear early in [22] with the Hilbert space subfamily $b_{q}^{2}$. Then the two-parameter family appears in [23]. In these two sources, the spaces are defined on general bounded domains given by smooth defining functions. In [24], a three-parameter family on $\mathbb{B}$ is considered, but this does not introduce any new spaces other than the two-parameter family precisely because of its Theorem 3 . It is interesting that $[22-26]$ do not deal with the case $p=1$.

More recently harmonic Besov spaces are studied in restricted ranges of the parameters, sometimes as a one-parameter subfamily. For example, [15] fixes $q$ at $q=-n$, but this reference is interesting in that it uses derivatives of integer order $t$ satisfying (1.2). In other places, only first-order derivatives are employed, and hence only small portions of the two-parameter Besov family are investigated. In [35], again $q=-n$ with $t=1$, so the values of $p$ that can be used are very limited. In $[39,43], q$ depends on $p$ with $t=1$, but in any case $q+p>-1$.

In this section, we note some properties of the $b_{q}^{p}$ that can be obtained directly from their definition. By (3.7), every $b_{q}^{p}$ contains all harmonic polynomials and thus is nontrivial. When $q>-1$, the finiteness of the measures $\nu_{q}$ shows that $h^{\infty} \subset b_{q}^{p}$. Since $D_{s}^{t} 1=1$ always, $\|\cdot\|_{b_{q}^{p}}$ is a true norm in all cases meaning that only $0 \in b_{q}^{p}$ has norm 0 .

Remark 4.1. Equivalently, if $q \leq-1$, the Besov space $b_{q}^{p}$ is defined as the space of all $u \in h(\mathbb{B})$ for which $D_{0}^{-q / p} u$ belongs to the Bergman space $b_{0}^{p}$, and if $q>-1$, it is simply defined as the weighted Bergman space $b_{q}^{p}$ since $I_{s}^{0}=I$ for any $s$. Explicitly,

$$
\|u\|_{b_{q}^{p}}^{p}= \begin{cases}\left\|D_{0}^{-q / p} u\right\|_{b_{0}^{p}}^{p}=\int_{\mathbb{B}}\left|D_{0}^{-q / p} u\right|^{p} d \nu & \text { if } q \leq-1 ; \\ \|u\|_{L_{q}^{p}}^{p}=\int_{\mathbb{B}}|u|^{p} d \nu_{q} & \text { if } q>-1 .\end{cases}
$$


So under (1.1), $I_{s}^{t}: b_{q}^{p} \rightarrow L_{q}^{p}$ is an isometric imbedding. Note that $t=-q / p>0$ for $q \leq-1$. Then for $q \leq-1$, by $(3.9)$, both $D_{0}^{-q / p}: b_{q}^{p} \rightarrow b_{0}^{p}$ and $D_{-q / p}^{q / p}: b_{0}^{p} \rightarrow b_{q}^{p}$ are isometric isomorphisms.

If $u \in \mathcal{H}_{m}$, then by (4.1), Definition 3.2, the polar coordinates formula, homogeneity, the beta integral, (3.8), and (2.1),

$$
\begin{aligned}
\|u\|_{b_{q}^{p}} & =\left(\frac{n}{V_{q}} d_{m}^{p}(s, t) \int_{0}^{1} r^{n-1+p m}\left(1-r^{2}\right)^{q+p t} \int_{\mathbb{S}}|u(\xi)|^{p} d \sigma(\xi) d r\right)^{1 / p} \\
& =\left(\frac{n}{2 V_{q}}\right)^{1 / p} d_{m}(s, t)\left(\frac{\Gamma((n+p m) / 2) \Gamma(1+q+p t)}{\Gamma(1+q+p t+(n+p m) / 2)}\right)^{1 / p}\|u\|_{L^{p}(\sigma)} \\
& \sim \frac{1}{m^{(1+q) / p}}\|u\|_{L^{p}(\sigma)},
\end{aligned}
$$

in which $q+p t=0$ and $s=0$ if $q \leq-1$, and $t=0$ if $q>-1$ by (1.1).

Theorem 4.1. Harmonic Besov spaces are complete and thus Banach spaces.

Proof. The completeness of the harmonic Bergman spaces is rather standard as they are closed subspaces of Lebesgue classes. A proof for the unweighted ones $b_{0}^{p}$ can be found in [1, Proposition 8.3], which works equally well for the weighted ones $b_{q}^{p}, q>-1$, too. The $b_{q}^{p}$ for $q \leq-1$ are also complete, because they are isometrically isomorphic to $b_{0}^{p}$ as noted in Remark 4.1.

Theorem 4.2. Harmonic polynomials and hence also $h(\overline{\mathbb{B}})$ are dense in every $b_{q}^{p}$. In particular, the dilates $u_{\tau}$ of $u \in b_{q}^{p}$ converge to $u$ in $b_{q}^{p}$ as $\tau \rightarrow 1^{-}$.

Proof. For the particular Bergman space $b_{0}^{2}$, this result is [1, Lemma 8.8]. Essential ingredients of its proof are the dilates, density of continuous functions on $\overline{\mathbb{B}}$ in Lebesgue classes, and the proof of Theorem 14.1 there. Thus the same proof works for all Bergman spaces $b_{q}^{p}, q>-1$.

In the proper Besov zone $q \leq-1$, if $u \in b_{q}^{p}$, we let $v=D_{s}^{t} u \in b_{0}^{p}$, where $s, t$ satisfy (1.1). If a harmonic polynomial $h$ approximates $v$ in $b_{0}^{p}$, then $D_{s+t}^{-t} h$ is also a harmonic polynomial by (3.7) and approximates $u$ in $b_{q}^{p}$ by Remark 4.1. Also if $g$ is harmonic on $\varepsilon \mathbb{B}$ with $\varepsilon>1$ and approximates $v$ in $b_{0}^{p}$, then $D_{s+t}^{-t} g$ is harmonic on the same ball by Theorem 3.1 and approximates $u$ in $b_{q}^{p}$ again by Remark 4.1 .

The claim about the dilates is inherent in [1, Lemma 8.8], and so is correct for the $b_{q}^{p}$ with $q>-1$. Then it is also correct for the $b_{q}^{p}$ with $q \leq-1$ by the previous paragraph.

Theorem 4.3. Harmonic Besov spaces are separable.

Proof. First let $q>-1$, take a $u \in b_{q}^{p}$, and let $\varepsilon>0$. Let $A_{m}$ be the set of all finite linear combinations of the members of the basis $\left\{Y_{m k}: k=1, \ldots, \delta_{m}\right\}$ of $\mathcal{H}_{m}$ with 
complex coefficients with rational real and imaginary parts, and let $A=\bigcup_{m=0}^{\infty} A_{m}$. Then the $A_{m}$ and $A$ are countable sets. Members of $A$ are harmonic polynomials. By Theorem 4.2, there is a harmonic polynomial $h$ such that $\|u-h\|_{b_{q}^{p}}<\varepsilon / 2$. The polynomial $h$ can be written as a finite linear combination of the $Y_{m k}$, each of which is bounded on $\mathbb{B}$. By approximating the coefficient of each term in the linear combination by a complex rational number, we can find a polynomial $g \in A$ such that $\|h-g\|_{L^{\infty}}<\varepsilon / 2$. Then also $\|h-g\|_{b_{q}^{p}}<\varepsilon / 2$ by the finiteness of the measure $\nu_{q}$ now. Thus $\|u-g\|_{b_{q}^{p}}<\varepsilon$, and $A$ is dense in $b_{q}^{p}$ for $q>-1$.

For $q \leq-1$, we use the isometries between $b_{q}^{p}$ and $b_{0}^{p}$ mentioned in Remark 4.1. Since $D_{-q / p}^{q / p}$ applied to a polynomial gives another polynomial with the same number of terms by definition, we obtain that $D_{-q / p}^{q / p} A$ is dense in $b_{q}^{p}$ for $q \leq-1$.

Proposition 4.1. Norm convergence in $b_{q}^{p}$ implies uniform convergence on compact subsets of $\mathbb{B}$.

Proof. Suppose $u^{j} \rightarrow u$ in $b_{q}^{p}$. With the $s, t$ of (1.1), this means $D_{s}^{t} u^{j} \rightarrow D_{s}^{t} u$ in $b_{q+p t}^{p}$, which is a weighted Bergman space. Since our weights are radial, [1, Proposition 8.1] is equally valid in weighted Bergman spaces. Then a standard argument shows that $D_{s}^{t} u^{j} \rightarrow D_{s}^{t} u$ uniformly on compact subsets of $\mathbb{B}$. By (3.9) and Theorem 3.2 , we obtain that $u^{j} \rightarrow u$ uniformly on compact subsets of $\mathbb{B}$.

Proposition 4.2. If $q>-1$ and also for $b_{-1}^{2}=h^{2}$, uniform convergence on $\mathbb{B}$ implies convergence in $\|\cdot\|_{b_{q}^{p}}$. If $q<-1$ and $\left\{u^{j}\right\}$ is a sequence of harmonic functions on an open set containing $\overline{\mathbb{B}}$ converging uniformly on compact subsets, then $\left\{u^{j}\right\}$ converges in $\|\cdot\|_{b_{q}^{p}}$.

Proof. The first statement is obvious since the norms in those spaces do not contain any derivatives and the measures $\nu_{q}$ are finite. For the second statement, the uniform convergence of $\left\{u^{j}\right\}$ on $\overline{\mathbb{B}}$ implies the uniform convergence of $\left\{D_{s}^{t} u^{j}\right\}$ on $\overline{\mathbb{B}}$ by Theorem 3.2. The form of $\|\cdot\|_{b_{q}^{p}}$ in (4.1) yields the desired conclusion since the measure in that norm is finite again.

\section{Hilbert Harmonic Besov Spaces}

In this section, we present results that are specific to those harmonic Besov spaces that are also Hilbert spaces, detailed results that can be derived from the presence of inner products and orthogonal bases. The Hilbert spaces are precisely those $b_{q}^{p}$ with $p=2$.

Let us first insert an identity that we need a few times in computations

$$
n \int_{0}^{1} r^{n-1+2 m}\left(1-r^{2}\right)^{q} d r=\frac{V_{q}}{\gamma_{m}(q)} \quad(q>-1, m \in \mathbb{N}) .
$$

Its derivation involves nothing but the beta integral and is thus omitted. 
Polarizing (4.1), we find inner products for the $b_{q}^{2}$ that make them Hilbert spaces. For $s, t$ as in (1.1) with $p=2$,

$$
[u, v]_{b_{q}^{2}}:=\left[I_{s}^{t} u, I_{s}^{t} v\right]_{L_{q}^{2}}= \begin{cases}\int_{\mathbb{B}} D_{0}^{-q / 2} \overline{u D_{0}^{-q / 2} v d \nu} & \text { if } q \leq-1 \\ \int_{\mathbb{B}} u \bar{v} d \nu_{q} & \text { if } q>-1 .\end{cases}
$$

If $u \in \mathcal{H}_{m}$ and $v \in \mathcal{H}_{l}$ in particular, we have by (5.2), Definition 3.2, the polar coordinates formula, and homogeneity,

$$
[u, v]_{b_{q}^{2}}=d_{m}(s, t) d_{l}(s, t) \frac{n}{V_{q}} \int_{0}^{1} r^{n-1+m+l}\left(1-r^{2}\right)^{q+2 t} \int_{\mathbb{S}} u(\xi) \overline{v(\xi)} d \sigma(\xi) d r .
$$

Continuing, if also $m=l$, then by (5.1) we have

$$
[u, v]_{b_{q}^{2}}=\frac{d_{m}(s, t)^{2}}{\gamma_{m}(q+2 t)}[u, v]_{L^{2}(\sigma)}=: N_{m}(q)[u, v]_{L^{2}(\sigma)},
$$

where

$$
N_{m}(q)=\left\{\begin{array}{ll}
\frac{\gamma_{m}(-q / 2)^{2}}{\gamma_{m}(0)^{3}} & \text { if } q \leq-1 \\
\frac{1}{\gamma_{m}(q)} & \text { if } q>-1
\end{array}\right\} \sim \frac{1}{m^{1+q}},
$$

because $q+2 t=0$ and $s=0$ if $q \leq-1$ and $t=0$ if $q>-1$ by (1.1), and by (3.5) and (3.8). In particular, $\left\|Y_{m k}\right\|_{b_{q}^{2}}^{2}=N_{m}(q)$ for every $k=1, \ldots, \delta_{m}$ since $\left\|Y_{m k}\right\|_{L^{2}(\sigma)}=1$.

Fix $x \in \mathbb{B}$. By the orthonormality of $\left\{Y_{m k}\right\}$ in $L^{2}(\sigma)$, homogeneity, and (14.7), we have

$$
\left\|Z_{m}(x, \cdot)\right\|_{L^{2}(\sigma)}^{2}=\sum_{k=1}^{\delta_{m}}\left|Y_{m k}(x)\right|^{2}=Z_{m}(x, x)=r^{2 m} Z_{m}(\xi, \xi)=\delta_{m} r^{2 m} .
$$

It follows that

$$
\left\|Z_{m}(x, \cdot)\right\|_{b_{q}^{2}}^{2}=N_{m}(q) \delta_{m} r^{2 m} \sim m^{n-3-q} r^{2 m}
$$

by (5.4), (5.5), and (14.1).

Proposition 5.1. If $m \neq l$, then $\mathcal{H}_{m}$ is orthogonal to $\mathcal{H}_{l}$ with respect to $[\cdot, \cdot]_{b_{q}^{2}}$. Further, if $u \in \mathcal{H}_{m}$ and $m>0$, then

$$
\int_{\mathbb{B}} u d \nu_{q}=0 \quad(q>-1)
$$

Proof. The first statement follows from (5.3) and the well-known mutual orthogonality (14.2) of the $\mathcal{H}_{m}$ in $L^{2}(\sigma)$. The integral in the second statement is just $[u, 1]_{b_{q}^{2}}$ for $q>-1$. 
Theorem 5.1. Suppose $u \in b_{q}^{2}$ has the expansions (14.9) and (14.11). Then

$$
\|u\|_{b_{q}^{2}}^{2}=\sum_{m=0}^{\infty}\left\|u_{m}\right\|_{b_{q}^{2}}^{2}=\sum_{m=0}^{\infty} N_{m}(q)\left\|u_{m}\right\|_{L^{2}(\sigma)}^{2}=\sum_{m=0}^{\infty} N_{m}(q) \sum_{k=1}^{\delta_{m}}\left|c_{m k}\right|^{2},
$$

where $s, t$ are as in (1.1). And $u \in h(\mathbb{B})$ belongs to $b_{q}^{2}$ if and only if

$$
\sum_{m=1}^{\infty} \frac{1}{m^{1+q}}\left\|u_{m}\right\|_{L^{2}(\sigma)}^{2}=\sum_{m=1}^{\infty} \frac{1}{m^{1+q}} \sum_{k=1}^{\delta_{m}}\left|c_{m k}\right|^{2}<\infty .
$$

Further, if $u \in b_{q}^{2}$, then both (14.9) and (14.11) converge to $u$ in $b_{q}^{2}$.

Let us remark that the case $q=-1$ corresponds to the harmonic Hardy space $h^{2}$ and follows immediately from (14.14).

Proof. Recall that $q+2 t=0$ and $s=0$ if $q \leq-1$, and $t=0$ if $q>-1$. We first write the integral in (5.2) over $\varepsilon \mathbb{B}$ and then let $\varepsilon \rightarrow 1^{-}$as explained right after the polar coordinates formula in Sec. 2. The homogeneous expansion converges uniformly on $\varepsilon \mathbb{B}$, so we can exchange the order of integration on this ball and sums of the homogeneous expansion. Since orthogonality is deduced from an integral on $\mathbb{S}$, Proposition 5.1 applied on $\varepsilon \mathbb{B}$ reduces the expression to a single sum of terms of the form (5.4). Then we use monotone convergence theorem to exchange the order of limit as $\varepsilon \rightarrow 1^{-}$and summation. In formulas, these mean

$$
\begin{aligned}
\|u\|_{b_{q}^{2}}^{2} & =\lim _{\varepsilon \rightarrow 1^{-}} \frac{1}{V_{q}} \int_{\varepsilon \mathbb{B}} \sum_{m=0}^{\infty} d_{m}(s, t) u_{m}(x) \sum_{l=0}^{\infty} d_{l}(s, t) \overline{u_{l}(x)}\left(1-|x|^{2}\right)^{q+2 t} d \nu(x) \\
& =\lim _{\varepsilon \rightarrow 1^{-}} \sum_{m=0}^{\infty} \frac{1}{V_{q}} \int_{\varepsilon \mathbb{B}} d_{m}(s, t)^{2}\left|u_{m}(x)\right|^{2}\left(1-|x|^{2}\right)^{q+2 t} d \nu(x) \\
& =\sum_{m=0}^{\infty} \frac{1}{V_{q}} \int_{\mathbb{B}} d_{m}(s, t)^{2}\left|u_{m}(x)\right|^{2}\left(1-|x|^{2}\right)^{q+2 t} d \nu(x)=\sum_{m=0}^{\infty}\left\|u_{m}\right\|_{b_{q}^{2}}^{2} .
\end{aligned}
$$

The inner sum over $k$ is handled similarly using the orthogonality of $\left\{Y_{m k}\right\}$; now we do not need to justify the exchange of order of various operations since this sum is finite. Along with (5.4), these considerations prove all the claims except the last. The convergence of the series for $\|u\|_{b_{q}^{2}}^{2}$ yields that

$$
\left\|u-\sum_{m=0}^{M-1} u_{m}\right\|_{b_{q}^{2}}^{2}=\left\|\sum_{m=M}^{\infty} u_{m}\right\|_{b_{q}^{2}}^{2}=\sum_{m=M}^{\infty}\left\|u_{m}\right\|_{b_{q}^{2}}^{2} \rightarrow 0 \quad(M \rightarrow \infty),
$$

which is the last claim for (14.9). This claim for (14.11) is identical.

We are led to a decomposition of every $b_{q}^{2}$ much like the decomposition in (14.2).

Corollary 5.1. For every $q \in \mathbb{R}, b_{q}^{2}=\bigoplus_{m=0}^{\infty} \mathcal{H}_{m}$, where convergence is in $\|\cdot\|_{b_{q}^{2}}$. 
Corollary 5.2. Theorem 1.1 holds true with $p=2$. More precisely, each selection of $t$ satisfying (1.2) with $p=2$ and of $s_{1}$ and $s_{2}$ gives rise to an equivalent norm

$$
\|u\|_{b_{q}^{2}}^{2}=\frac{1}{V_{q}} \int_{\mathbb{B}} D_{s_{1}}^{t} u(x) \overline{D_{s_{2}}^{t} u(x)}\left(1-|x|^{2}\right)^{q+2 t} d \nu(x)
$$

on $b_{q}^{2}$.

Remark 5.1. All the computations around (5.3)-(5.5) make sense for any $s_{1}, s_{2}, t$ satisfying (1.2) and yield the same order of growth for $N_{m}(q)$.

Proof. Let $u \in b_{q}^{2}$. By Remark 5.1 and Theorem 5.1,

$$
\|u\|_{b_{q}^{2}}^{2} \sim 1+\sum_{m=1}^{\infty} \frac{1}{m^{1+q}}\|u\|_{L^{2}(\sigma)}^{2}
$$

independently of the $s, t$ used in $\|\cdot\|_{b_{q}^{2}}$.

Now polarizing (5.8), we find a whole family of inner products

$$
[u, v]_{b_{q}^{2}}=\left[I_{s_{1}}^{t} u, I_{s_{2}}^{t} v\right]_{L_{q}^{2}}=\frac{1}{V_{q}} \int_{\mathbb{B}} D_{s_{1}}^{t} u(x) \overline{D_{s_{2}}^{t} v(x)}\left(1-|x|^{2}\right)^{q+2 t} d \nu(x)
$$

for $b_{q}^{2}$. These inner products are equivalent in the sense that the norms they induce are equivalent.

We can finally form the promised connection between the kernels of Definition 3.1 and the spaces of Definition 1.1 and prove Theorem 1.3. We restate it explicitly for convenience.

Theorem 5.2. Given a $q \in \mathbb{R}$, there are $t, s_{1}, s_{2}$ such that for any $u \in b_{q}^{2}$ and $x \in \mathbb{B}$, we have

$$
u(x)=\left[u(\cdot), R_{q}(x, \cdot)\right]_{b_{q}^{2}}=\frac{1}{V_{q}} \int_{\mathbb{B}} D_{s_{1}}^{t} u(y) D_{s_{2}}^{t} R_{q}(x, y)\left(1-|y|^{2}\right)^{q+2 t} d \nu(y) .
$$

Proof of Theorems $\mathbf{1 . 3}$ and 5.2. The proof works equally well in the proper Besov zone $q \leq-1$ and the Bergman zone $q>-1$.

Fix $x \in \mathbb{B}$. We know $R_{q}(x, y)$ is harmonic in $y \in \mathbb{B}$. It also follows from (5.6) and (3.5) that the series in $(3.3)$ defining $R_{q}(x, \cdot)$ converges in $b_{q}^{2}$ by the same reason as for $(3.6)$. Hence $R_{q}(x, \cdot) \in b_{q}^{2}$ by Theorem 5.1. This could be proved also via Theorem 4.2 .

Pick $t$ such that $q+2 t=0$ for $q \leq-1$ and $t=0$ for $q>-1$. In any case $q_{1}=q+2 t>-1$ and (1.2) is satisfied with $p=2$. Let $s_{1}=q+t$ and $s_{2}=q$. Initially let $u \in \mathcal{H}_{m}$. By orthogonality, (5.9), Definition 3.2, (5.4), Remark 5.1, and (14.4), we obtain

$$
\begin{aligned}
{\left[u(\cdot), R_{q}(x, \cdot)\right]_{b_{q}^{2}} } & =\left[u(\cdot), \gamma_{m}(q) Z_{m}(x, \cdot)\right]_{b_{q}^{2}}=\left[I_{s_{1}}^{t} u(\cdot), \gamma_{m}(q) I_{s_{2}}^{t} Z_{m}(x, \cdot)\right]_{L_{q}^{2}} \\
& =d_{m}\left(s_{1}, t\right) d_{m}\left(s_{2}, t\right) \frac{\gamma_{m}(q)}{V_{q}} \int_{\mathbb{B}} u(y) Z_{m}(x, y)\left(1-|y|^{2}\right)^{q_{1}} d \nu(y)
\end{aligned}
$$




$$
\begin{aligned}
& =\gamma_{m}\left(q_{1}\right) \frac{1}{V_{q}} \frac{V_{q_{1}}}{\gamma_{m}\left(q_{1}\right)}\left[u(\cdot), Z_{m}(x, \cdot)\right]_{L^{2}(\sigma)} \\
& =\frac{V_{q+2 t}}{V_{q}} u(x)=u(x)
\end{aligned}
$$

by the way $s_{1}, s_{2}$, and the $V_{q}$ are chosen.

The same result holds also for $u$ a finite sum of elements of the $\mathcal{H}_{m}$ and hence for any harmonic polynomial $u$. If $u \in b_{q}^{2}$ is arbitrary, then $u$ can be approximated in $\|\cdot\|_{b_{q}^{2}}$ by a sequence $\left\{u^{j}\right\}$ of harmonic polynomials by Theorem 4.2. This implies by the Cauchy-Schwarz inequality that $\left[u^{j}(\cdot), R_{q}(x, \cdot)\right]_{b_{q}^{2}} \rightarrow\left[u(\cdot), R_{q}(x, \cdot)\right]_{b_{q}^{2}}$ as $j \rightarrow \infty$ for each $x \in \mathbb{B}$. By Proposition 4.1, also $u^{j}(x) \rightarrow u(x)$ as $j \rightarrow \infty$ for each $x \in \mathbb{B}$. Therefore the reproducing property holds for all $u \in b_{q}^{2}$.

Proposition 5.2. If $q_{1}<q_{2}$, then $b_{q_{1}}^{2} \subset b_{q_{2}}^{2}$ without being equal, and the inclusion map $i: b_{q_{1}}^{2} \rightarrow b_{q_{2}}^{2}$ is compact.

Proof. The set inclusion follows immediately from (5.7). For a counterexample to equality, define

$$
u(x)=\sum_{m=1}^{\infty} m^{\left(q_{1}+q_{2}\right) / 4} Y_{m 1}(x)
$$

Again by (5.7),

$$
\|u\|_{q_{1}}^{2} \sim \sum_{m=1}^{\infty} \frac{1}{m^{1-\left(q_{2}-q_{1}\right) / 2}}=\infty \quad \text { while }\|u\|_{q_{2}}^{2} \sim \sum_{m=1}^{\infty} \frac{1}{m^{1+\left(q_{2}-q_{1}\right) / 2}}<\infty .
$$

Hence $u \in b_{q_{2}}^{2} \backslash b_{q_{1}}^{2}$.

Next, considering the homogeneous expansion of $u$, for $M=1,2, \ldots$, define maps $i_{M}: b_{q_{1}}^{2} \rightarrow b_{q_{2}}^{2}$ by $i_{M}(u)=\sum_{m=0}^{M-1} u_{m}$. Each $i_{M}$ has finite rank since the $\mathcal{H}_{m}$ are finite-dimensional. Theorem 5.1 yields

$$
\begin{aligned}
\left\|\left(i-i_{M}\right) u\right\|_{b_{q_{2}}^{2}}^{2} & \sim \sum_{m=M}^{\infty} \frac{1}{m^{1+q_{2}}}\left\|u_{m}\right\|_{L^{2}(\sigma)}^{2}=\sum_{m=M}^{\infty} \frac{1}{m^{1+q_{1}}} \frac{1}{m^{q^{2}-q_{1}}}\left\|u_{m}\right\|_{L^{2}(\sigma)}^{2} \\
& \leq \frac{1}{M^{q_{2}-q_{1}}} \sum_{m=1}^{\infty} \frac{1}{m^{1+q_{1}}}\left\|u_{m}\right\|_{L^{2}(\sigma)}^{2} \sim \frac{1}{M^{q_{2}-q_{1}}}\|u\|_{b_{q_{1}}^{2}}^{2} .
\end{aligned}
$$

Thus $\left\|i-i_{M}\right\| \lesssim M^{-\left(q_{2}-q_{1}\right) / 2} \rightarrow 0$ as $M \rightarrow \infty$. Therefore $i$ is compact being a norm limit of operators of finite rank.

Proposition 5.3. For any $s, t$, the map $D_{s}^{t}: b_{q}^{2} \rightarrow b_{q+2 t}^{2}$ is a bijection with inverse $D_{s+t}^{-t}$. If $q_{1}+2 t<q_{2}$, then for any $s, D_{s}^{t}: b_{q_{1}}^{2} \rightarrow b_{q_{2}}^{2}$ is compact.

Proof. Both claims follow in a manner similar to the proof of Proposition 5.2 using Definition 3.2, (3.8), (3.9), and (5.7). 
Corollary 5.2 and Propositions 5.2 and 5.3 on the Hilbert spaces $b_{q}^{2}$ are later generalized to all $b_{q}^{p}$ spaces.

Besov spaces are defined by imbeddings into the Lebesgue classes $L_{q}^{p}$, and for another Lebesgue class $L^{2}(\sigma)$ we have the decomposition (14.2). We now answer the question whether there is a similar decomposition for $L_{q}^{2}$ too by generalizing the case $q=0$ taken care of in [38, Lemma IV.2.18].

Definition 5.1. We define the space $\mathcal{G}_{m}(q)$ as the span of functions of the form $g(x)=g(r \xi)=f(r) u(\xi)$, where $f$ is a measurable radial function for $0 \leq r<1$ and $u \in \mathcal{H}_{m}$ in such a way that $g \in L_{q}^{2}$.

Theorem 5.3. For every $q \in \mathbb{R}$, we have the orthogonal direct sum decomposition $L_{q}^{2}=\bigoplus_{m=0}^{\infty} \mathcal{G}_{m}(q)$.

Proof. Mutual orthogonality of the $\mathcal{G}_{m}(q)$ in $L_{q}^{2}$ follows from that of the $\mathcal{H}_{m}$ in $L^{2}(\sigma)$ as in Proposition 5.1.

Next consider the orthonormal basis for $\mathcal{H}_{m}$ in (14.3). A typical $g \in \mathcal{G}_{m}(q)$ can be written in the form $g(x)=f_{1}(r) Y_{m 1}(\xi)+\cdots+f_{\delta_{m}}(r) Y_{m \delta_{m}}(\xi)$ for $x \in \mathbb{B}$. By the polar coordinates formula and the orthonormality of $\left\{Y_{m k}\right\}$, we have

$$
\|g\|_{L_{q}^{2}}^{2}=\frac{n}{V_{q}} \sum_{k=1}^{\delta_{m}} \int_{0}^{1}\left|f_{k}(r)\right|^{2} r^{n-1}\left(1-r^{2}\right)^{q} d r<\infty .
$$

This formula shows that if a sequence $\left\{g^{j}\right\}$ in $\mathcal{G}_{m}(q)$ converges to $g \in L_{q}^{2}$, then the corresponding sequences $\left\{f_{k}^{j}\right\}$ converge to, say, $f_{k}$ as $j \rightarrow \infty$ in $L^{2}[0,1)$ with respect to the measure $r^{n-1}\left(1-r^{2}\right)^{q} d r$. Then the $g$ formed with the limit $f_{k}$ is the limit of $\left\{g^{j}\right\}$ in $L_{q}^{2}$, and such a $g$ belongs to $\mathcal{G}_{m}(q)$ by the way it is formed. Thus $\mathcal{G}_{m}(q)$ is a closed subspace of $L_{q}^{2}$.

Suppose $h \in L_{q}^{2}$ is orthogonal to all the $\mathcal{G}_{m}(q), m=0,1,2, \ldots$ If $g \in \mathcal{G}_{m}(q)$ is like above, this means that

$$
\sum_{k=1}^{\delta_{m}} \int_{0}^{1} \overline{f_{k}(r)} \int_{\mathbb{S}} h_{r}(\xi) \overline{Y_{m k}(\xi)} d \sigma(\xi) r^{n-1}\left(1-r^{2}\right)^{q} d r=0 .
$$

Taking $f_{k}(r)$ equal to the integral over $\mathbb{S}$ above, we see that the integral is 0 for a.e. $r \in[0,1)$, that is, almost every dilate $h_{r}$ of $h$ is orthogonal to every basis element $Y_{m k}$ in every $\mathcal{H}_{m}$. Thus for a.e. $r \in(0,1), h_{r}(\xi)=0$ for a.e. $\xi \in \mathbb{S}$ by (14.2). Then $h=0$ a.e. on $\mathbb{B}$. Thus $L_{q}^{2}$ decomposes the way it is stated.

As done at the end of Sec. 14, let us have a look at the case $n=2$ again. Then $Y_{m 1}(x)=x^{m}, Y_{m 2}(x)=\bar{x}^{m}$, and the expansion (14.11) takes the form

$$
u(x)=\sum_{m=-\infty}^{\infty} c_{m} r^{|m|} e^{i m \theta}
$$


which is nothing but the Abel means of the Fourier series of $u$ whenever $u$ has boundary values. The coefficients are

$$
c_{m}=\frac{1}{2 \pi r^{|m|}} \int_{-\pi}^{\pi} u\left(r e^{i \theta}\right) e^{-i m \theta} d \theta=\frac{1}{2 \pi} \int_{-\pi}^{\pi} u\left(e^{i \theta}\right) e^{-i m \theta} d \theta,
$$

where the second form is valid if $u$ has boundary values which is the case due to $\left[1\right.$, p. 137] when $u \in b_{q}^{2}$ with $q \leq-1$ since then $b_{q}^{2} \subset b_{-1}^{2}=h^{2}$ by Proposition 5.2. Moreover, $u \in h(\mathbb{B})$ belongs to $b_{q}^{2}$ if and only if

$$
\sum_{m=-\infty}^{\infty} N_{|m|}(q)\left|c_{m}\right|^{2} \sim \sum_{\substack{m=-\infty \\ m \neq 0}}^{\infty} \frac{\left|c_{m}\right|^{2}}{|m|^{1+q}}<\infty
$$

which reduces to the expected result that $u \in h^{2}$ if and only if $\sum_{m=-\infty}^{\infty}\left|c_{m}\right|^{2}<\infty$.

\section{Preparatory Calculations}

In this section, we collect some results used extensively in the proofs of the theorems in the next two sections. The lemmas are for the estimates of the kernels in Sec. 7 and are mostly known in the case $c>0$. Here we supply the missing cases $c \leq 0$ and the full proofs not only for completeness, but also for some simplifications and making some remarks. At the end we prove a Schur test that fits better the proof of the characterizations of the integral transforms in Sec. 8.

One way to define the Gegenbauer (ultraspherical) polynomials $G_{m}^{d}$ of degree $m$ is via $G_{0}^{0}=1$ and the generating functions

$$
\frac{1}{\left(1-2 \zeta \tau+\tau^{2}\right)^{d}}=\sum_{m=0}^{\infty} G_{m}^{d}(\zeta) \tau^{m} \quad(d>-1 / 2, d \neq 0)
$$

and

$$
\log \frac{1}{1-2 \zeta \tau+\tau^{2}}=\sum_{m=1}^{\infty} G_{m}^{0}(\zeta) \tau^{m}
$$

where convergence is assured for $|\zeta| \leq 1$ and $|\tau|<1$. Specifically, $G_{m}^{1}(\zeta)=U_{m}(\zeta)$ and $G_{m}^{0}(\zeta)=2 T_{m}(\zeta) / m$, where the $U_{m}$ and the $T_{m}(\zeta)=\cos \left(m \cos ^{-1} \zeta\right)$ are the Chebyshev polynomials of the second and first kinds, respectively. Gegenbauer polynomials have the special values

$$
G_{m}^{d}(1)=\frac{(2 d)_{m}}{m !} \sim m^{2 d-1} \quad(d \neq 0) \quad \text { and } \quad G_{m}^{0}(1)=\frac{2}{m} .
$$

The material in this paragraph is taken from [30, Chap. 18].

Lemma 6.1. For $a>-1, b>-1, c \in \mathbb{R}, x=r \xi \in \mathbb{B}$, and $\eta \in \mathbb{S}$,

$$
\int_{0}^{1} \frac{\lambda^{a}\left(1-\lambda^{2}\right)^{b} d \lambda}{\left\{\lambda^{2} x, \eta\right\}^{(1+b+c) / 2}}=\int_{0}^{1} \frac{\lambda^{a}\left(1-\lambda^{2}\right)^{b} d \lambda}{\left|\lambda^{2} x-\eta\right|^{1+b+c}} \sim \begin{cases}1 & \text { if } c<0 \\ 1+\log |x-\eta|^{-1} & \text { if } c=0 \\ |x-\eta|^{-c} & \text { if } c>0 .\end{cases}
$$


Proof. Call the integral $M$ and put $2 d=1+b+c$. If $d \leq 0, M$ is clearly bounded. If $d>0$, we use the generating functions of Gegenbauer polynomials to expand the denominator and then compute using the beta integral:

$$
\begin{aligned}
M & =\int_{0}^{1} \frac{\lambda^{a}\left(1-\lambda^{2}\right)^{b} d \lambda}{\left(1-2\left(\lambda^{2} r\right) \xi \cdot \eta+\left(\lambda^{2} r\right)^{2}\right)^{d}} \\
& =\int_{0}^{1} \lambda^{a}\left(1-\lambda^{2}\right)^{b} \sum_{m=0}^{\infty} G_{m}^{d}(\xi \cdot \eta)\left(\lambda^{2} r\right)^{m} d \lambda \\
& =\sum_{m=0}^{\infty} G_{m}^{d}(\xi \cdot \eta) r^{m} \int_{0}^{1} \lambda^{a+2 m}\left(1-\lambda^{2}\right)^{b} d \lambda \\
& =\sum_{m=0}^{\infty} G_{m}^{d}(\xi \cdot \eta) r^{m} \frac{\Gamma(1 / 2+a / 2+m) \Gamma(1+b)}{2 \Gamma(3 / 2+a / 2+b+m)} .
\end{aligned}
$$

The only singularity of the integrand that needs to be studied is the one as $x \rightarrow \eta$; so without loss of generality we can take $x=r \eta$. Then $\xi \cdot \eta=1$, we can use $(6.2)$ and obtain

$$
M \sim 1+\sum_{m=1}^{\infty} \frac{1}{m^{1+b}} \frac{(2 d)_{m}}{m !} r^{m} \sim 1+\sum_{m=1}^{\infty} m^{c-1} r^{m} .
$$

It is now clear that $M$ is bounded if $c<0$. If $c \geq 0$, then

$$
M \sim \sum_{m=0}^{\infty} G_{m}^{c / 2}(1) r^{m} \sim \sum_{m=0}^{\infty} G_{m}^{c / 2}(\xi \cdot \eta) r^{m} .
$$

The result mostly follows from the generating functions of the $G_{m}^{c / 2}$ and (2.4).

Last, when $c<0, M$ is bounded from below by the integral of the same integrand from $1 / 4$ to $1 / 2$. Then inequalities like $3 / 4 \leq\left|\lambda^{2} x-\eta\right| \leq 2$ show that $M$ is bounded away from 0 .

See also [6, Lemma 4.2; 14, Proof of Lemma 2.7; 33, Lemma 4.2; 28, Lemma 2.7] for more restricted versions.

Lemma 6.2. For $c \in \mathbb{R}, b>-1$, and $x=r \xi \in \mathbb{B}$,

$$
\left.\begin{array}{l}
\int_{\mathbb{S}} \frac{1}{\{x, \eta\}^{(n-1+c) / 2}} d \sigma(\eta) \\
\int_{\mathbb{B}} \frac{\left(1-|y|^{2}\right)^{b}}{\{x, y\}^{(n+b+c) / 2}} d \nu(y)
\end{array}\right\} \sim \begin{cases}1 & \text { if } c<0 \\
|x|^{-2} \log \left(1-|x|^{2}\right)^{-1} & \text { if } c=0 \\
\left(1-|x|^{2}\right)^{-c} & \text { if } c>0 .\end{cases}
$$

Proof. These are the estimates in [27, Proposition 2.2]. However the proof of the integral on $\mathbb{S}$ there requires clarification at two points.

First, for $n-1+c \leq 0$, the integral is clearly bounded, so the expansion of the denominator of the integrand in terms of Gegenbauer polynomials is necessary only for $n-1+c>0$. In fact, the expansion is not valid for all $n-1+c \in \mathbb{R}$. 
Second, after obtaining a sum involving ${ }_{2} F_{1}(-m, m+(n-1+c) / 2 ; n / 2 ; 1)$, this function value is computed using a reasoning that does not work for all values of $c$ under consideration, although the given value is right. The correct reason for this evaluation is the Chu-Vandermonde identity (2.8).

See also [14, Lemma 2.9] for a more restricted version and [33, Lemma 4.4] for a different proof. Also related is [5, Lemma 3.5].

Lemma 6.3. For $a>-1, b>-1, c \in \mathbb{R}$, and $0 \leq r<1$,

$$
\int_{0}^{1} \frac{\rho^{a}\left(1-\rho^{2}\right)^{b}}{\left(1-r^{2} \rho^{2}\right)^{1+b+c}} d \rho \sim \begin{cases}1 & \text { if } c<0 \\ r^{-2} \log \left(1-r^{2}\right)^{-1} & \text { if } c=0 \\ \left(1-r^{2}\right)^{-c} & \text { if } c>0\end{cases}
$$

Proof. Call the integral $M$. We compute using the beta integral and (2.1).

$$
\begin{aligned}
M & =\int_{0}^{1} \rho^{a}\left(1-\rho^{2}\right)^{b} \sum_{m=0}^{\infty} \frac{(1+b+c)_{m}}{m !}\left(r^{2} \rho^{2}\right)^{m} d \rho \\
& =\sum_{m=0}^{\infty} \frac{(1+b+c)_{m}}{m !} r^{2 m} \int_{0}^{1} \rho^{a+2 m}\left(1-\rho^{2}\right)^{b} d \rho \\
& =\sum_{m=0}^{\infty} \frac{(1+b+c)_{m}}{(1)_{m}} \frac{\Gamma(1 / 2+a / 2+m) \Gamma(1+b)}{2 \Gamma(3 / 2+a / 2+b+m)} r^{2 m} \\
& \sim 1+\sum_{m=1}^{\infty} m^{c-1} r^{2 m} .
\end{aligned}
$$

That $M$ is bounded away from 0 when $c<0$ is proved as in Lemma 6.1.

See also [6, Lemma 3.1; 14, Lemma 2.1; 37, Lemma 6] for more restricted versions.

Remark 6.1. In Lemmas 6.1 and 6.2, denoting the integrals $M(x)$, for $c<0$, we have $M(x) \sim 1+\sum_{m=1}^{\infty} m^{c-1}|x|^{m}$ in the proofs. Putting $|x|=1$, we notice that the sums are uniformly convergent for $x \in \overline{\mathbb{B}}$ and $M(x)$ extends continuously to all of $\overline{\mathbb{B}}$ by the Abel lemma. Similarly in Lemma 6.3, the integral extends continuously to $r=1$ when $c<0$.

The following is our version of the Schur test on the boundedness of integral transforms on Lebesgue classes. It involves a change of measure and the adjoint of the transform.

Theorem 6.1. Suppose $\mu, \kappa$ are $\sigma$-finite positive measures on the same $\sigma$-algebra on a set $A, \mu$ is absolutely continuous with respect to $\kappa$, and $T$ defined for a suitable $f$ by

$$
T f(x):=\int_{A} K(x, y) f(y) d \mu(y)
$$


is an integral operator with nonnegative measurable kernel $K$ defined on $A \times A$. Suppose further that $1<p<\infty$ and there is a strictly positive measurable function $h$ on $A$ such that $T\left(h^{p^{\prime}}\right)(x) \leq C_{1} h^{p^{\prime}}(x)$ a.e. $[\kappa]$ and $T^{*}\left(h^{p}\right)(y) \leq C_{2} h^{p}(y)$ a.e. $[\kappa]$ for some constants $C_{2}, C_{1}>0$, where $T^{*}$ is the formal adjoint of $T$ with respect to the pairing induced by the inner product of $L^{2}(\kappa)$. Then $T: L^{p}(\kappa) \rightarrow L^{p}(\kappa)$ is bounded.

Proof. The adjoint $T^{*}: L^{p^{\prime}}(\kappa) \rightarrow L^{p^{\prime}}(\kappa)$ of $T$ is obtained from

$$
\begin{aligned}
{[T f, g]_{L^{2}(\kappa)} } & =\int_{A} \int_{A} K(x, y) f(y) d \mu(y) \overline{g(x)} d \kappa(x) \\
& =\int_{A} f(y) \overline{\int_{A} K(x, y) g(x) d \kappa(x)} d \mu(y) \\
& =\int_{A} f(y) \overline{\frac{d \mu}{d \kappa}(y) \int_{A} K(x, y) g(x) d \kappa(x)} d \kappa(y)=\left[f, T^{*} g\right]_{L^{2}(\kappa)}
\end{aligned}
$$

as

$$
T^{*} g=\frac{d \mu}{d \kappa} \int_{A} K(x, \cdot) g(x) d \kappa(x),
$$

where $d \mu / d \kappa$ is the Radon-Nikodym derivative of $\mu$ with respect to $\kappa$, which exists under the assumptions on the measures. The positivity of $h$, Hölder inequality, and one assumption on $h$ first yield

$$
\begin{aligned}
|T f(x)| & \leq \int_{A} K(x, y) h(y) h(y)^{-1}|f(y)| d \mu(y) \\
& \leq\left(\int_{A} K(x, y) h(y)^{p^{\prime}} d \mu(y)\right)^{1 / p^{\prime}}\left(\int_{A} K(x, y) h(y)^{-p}|f(y)|^{p} d \mu(y)\right)^{1 / p} \\
& \lesssim h(x)\left(\int_{A} K(x, y) h(y)^{-p}|f(y)|^{p} d \mu(y)\right)^{1 / p} .
\end{aligned}
$$

The Fubini theorem and the other assumption on $h$ then give

$$
\begin{aligned}
\int_{A}|T f(x)|^{p} d \kappa(x) & \lesssim \int_{A}|h(x)|^{p} \int_{A} K(x, y) h(y)^{-p}|f(y)|^{p} d \mu(y) d \kappa(x) \\
& =\int_{A} h(y)^{-p}|f(y)|^{p} \int_{A} K(x, y)|h(x)|^{p} d \kappa(x) d \mu(y) \\
& =\int_{A} h(y)^{-p}|f(y)|^{p} \frac{d \mu}{d \kappa}(y) \int_{A} K(x, y)|h(x)|^{p} d \kappa(x) d \kappa(y) \\
& \lesssim \int_{A} h(y)^{-p}|f(y)|^{p} h(y)^{p} d \kappa(y)=\int_{A}|f|^{p} d \kappa .
\end{aligned}
$$

Checking the constants carefully shows that $\|T\| \leq C_{2}^{1 / p} C_{1}^{1 / p^{\prime}}$. 


\section{Estimates on Harmonic Besov Kernels}

The purpose of this section is to prove Theorem 1.5. This theorem is the harmonic counterpart of the well-known Forelli-Rudin estimates in [36, Proposition 1.4.10] on the integrals of holomorphic Bergman-Besov kernels. We see that the harmonic case is considerably more difficult than the holomorphic case mainly because the harmonic Bergman-Besov kernels are not powers of a simple binomial expression whose pointwise bounds are trivial. For the same reason, the verification of the upper and lower bounds has to pass through different paths.

We start with the following known result on Poisson and harmonic Bergman kernels.

Lemma 7.1. For $q \geq-1$ and $\alpha$ a multi-index, we have

$$
\left|\partial^{\alpha} R_{q}(x, \eta)\right| \lesssim \frac{1}{|x-\eta|^{n+q+|\alpha|}} \quad(x \in \mathbb{B}, \eta \in \mathbb{S}),
$$

where the partial derivatives are applied to the first variable, which is always the case in this section.

With $\alpha=(0, \ldots, 0)$ and $q=-1$, growth estimate of the Poisson kernel is included in this lemma.

Proof. This is a combination of [14, Lemmas 2.7 and 2.8]. In this reference, Lemma 2.8 is stated for $q>-1$, but it is true also for $q=-1$ since Lemma 2.7 on which it is based is stated and proved for $q \geq-1$. Note that our $q$ corresponds to their $\alpha-1$, while our $\alpha$ is a multi-index.

What we need are pointwise upper bounds on the Besov kernels $(q \in \mathbb{R})$ and another kernel of similar growth in Sec. 9. We also need control over the partial derivatives of the kernels for Sec. 12. So we shape our lemmas to accommodate greater generality.

Lemma 7.2. For $l=0,1,2, \ldots, b>0, x \in \mathbb{B}$, and $\eta \in \mathbb{S}$, let

$$
W_{l}(x, \eta)=\sum_{m=0}^{\infty} \frac{\Gamma(b+l+m)}{\Gamma(b+m)} Z_{m}(x, \eta) .
$$

Then for a multi-index $\alpha$,

$$
\left(\partial^{\alpha} W_{l}\right)(x, \eta) \mid \lesssim \frac{1}{|x-\eta|^{n-1+l+|\alpha|}} .
$$

Proof. First let $\alpha=(0, \ldots, 0)$. By homogeneity,

$$
\tau^{b+l-1} P(\tau x, \eta)=\sum_{m=0}^{\infty} \tau^{b+l-1+m} Z_{m}(x, \eta) .
$$


Then on the one hand,

$$
\left.\frac{\partial^{l}}{\partial \tau^{l}}\left(\tau^{b+l-1} P(\tau x, \eta)\right)\right|_{\tau=1}=\sum_{m=0}^{\infty}(b+m)_{l} Z_{m}(x, \eta)=W_{l}(x, \eta) .
$$

On the other hand, by the Leibniz and chain rules,

$$
\begin{aligned}
\left.\frac{\partial^{l}}{\partial \tau^{l}}\left(\tau^{b+l-1} P(\tau x, \eta)\right)\right|_{\tau=1} & =\left.\sum_{j=0}^{l}\left(\begin{array}{l}
l \\
j
\end{array}\right) \frac{d^{l-j}}{d \tau^{l-j}}\left(\tau^{b+l-1}\right) \frac{\partial^{j}}{\partial \tau^{j}}(P(\tau x, \eta))\right|_{\tau=1} \\
& =\left.\sum_{j=0}^{l}\left(\begin{array}{l}
l \\
j
\end{array}\right)(b+j)_{l-j} \sum_{|\beta|=j} \frac{j !}{\beta !} x^{\beta}\left(\partial^{\beta} P\right)(\tau x, \eta)\right|_{\tau=1}
\end{aligned}
$$

Since the largest value of $j$ and $|\beta|$ is $l$ and $|x|<1$, the desired upper bound on $W_{l}$ follows from the $q=-1$ case of Lemma 7.1.

Second, for any multi-index $\alpha$,

$$
\begin{aligned}
\left(\partial^{\alpha} W_{l}\right)(x, \eta) & =\left.\frac{\partial^{l}}{\partial \tau^{l}}\left(\tau^{b+l-1} \partial^{\alpha}(P(\tau x, \eta))\right)\right|_{\tau=1} \\
& =\left.\frac{\partial^{l}}{\partial \tau^{l}}\left(\tau^{b+l-1+|\alpha|}\left(\partial^{\alpha} P\right)(\tau x, \eta)\right)\right|_{\tau=1} .
\end{aligned}
$$

Now we repeat the calculation in (7.1) and obtain an expression that differs from the last one there in that it has $\partial^{\alpha+\beta}$ instead of $\partial^{\alpha}$ acting on $P$ with $|\beta+\alpha| \leq l+|\alpha|$. We resort to Lemma 7.1 again to finish the proof.

Now we remove the restriction that $l$ should be an integer. We follow the method of [14, Lemma 2.4].

Lemma 7.3. For $a, b>0, x \in \mathbb{B}$, and $\eta \in \mathbb{S}$, let

$$
W(x, \eta)=\sum_{m=0}^{\infty} \frac{\Gamma(a+m)}{\Gamma(b+m)} Z_{m}(x, \eta) .
$$

For a multi-index $\alpha$, put $c=n-1+a-b+|\alpha|$. Then

$$
\left|\left(\partial^{\alpha} W\right)(x, \eta)\right| \lesssim \begin{cases}1 & \text { if } c<0 \\ 1+\log |x-\eta|^{-1} & \text { if } c=0 \\ |x-\eta|^{-c} & \text { if } c>0 .\end{cases}
$$

Proof. Again first let $\alpha=(0, \ldots, 0)$. Choose a positive integer $l$ large enough that $b-a+l>0$. Using the homogeneity of the $Z_{m}$ and the beta integral, it is easy to check

$$
W(x, \eta)=\frac{2}{\Gamma(b-a+l)} \int_{0}^{1} W_{l}\left(\lambda^{2} x, \eta\right) \lambda^{2 a-1}\left(1-\lambda^{2}\right)^{b-a+l-1} d \lambda .
$$


It involves exchanging the order of integration and summation using the uniform convergence of $W_{l}\left(\lambda^{2} x, \eta\right)$ for $0 \leq \lambda \leq 1$ when $x \in \mathbb{B}$ is fixed using the same reasoning as in Proposition 3.1 since the coefficient of the $Z_{m}$ in $W_{l}$ grows like $m^{l}$. By Lemma 7.2,

$$
|W(x, \eta)| \lesssim \int_{0}^{1} \frac{\lambda^{2 a-1}\left(1-\lambda^{2}\right)^{b-a+l-1}}{\left|\lambda^{2} x-\eta\right|^{n-1+l}} d \lambda .
$$

Now the growth rates of $W(x, \eta)$ for all $c$ can be read off from Lemma 6.1.

Differentiation under the integral sign shows that

$$
\left(\partial^{\alpha} W\right)(x, \eta)=C \int_{0}^{1}\left(\partial^{\alpha} W_{l}\right)\left(\lambda^{2} x, \eta\right) \lambda^{2 a-1+2|\alpha|}\left(1-\lambda^{2}\right)^{b-a+l-1} d \lambda .
$$

After using Lemma 7.2, conclusions on $\partial^{\alpha} W$ can be read off from Lemma 6.1 once again.

Lemma 7.4. For $a_{j}, b_{j}>0, x \in \mathbb{B}$, and $\eta \in \mathbb{S}$, let

$$
W(x, \eta)=\sum_{m=0}^{\infty} \frac{\Gamma\left(a_{1}+m\right)}{\Gamma\left(b_{1}+m\right)} \cdots \frac{\Gamma\left(a_{k}+m\right)}{\Gamma\left(b_{k}+m\right)} Z_{m}(x, \eta) .
$$

For a multi-index $\alpha$, put $c=n-1+\left(a_{1}+\cdots+a_{k}\right)-\left(b_{1}+\cdots+b_{k}\right)+|\alpha|$. Then

$$
\left|\left(\partial^{\alpha} W\right)(x, \eta)\right| \lesssim \begin{cases}1 & \text { if } c<0 \\ 1+\log |x-\eta|^{-1} & \text { if } c=0 \\ |x-\eta|^{-c} & \text { if } c>0 .\end{cases}
$$

Proof. It is a matter of induction. In the induction step, in place of the Poisson kernel, we start with a kernel whose last factor in the coefficient of the $Z_{m}$ has the form of the coefficient of $W_{l}$, and repeat Lemmas 7.2 and 7.3.

We can finally give the pointwise upper bounds on the Besov kernels.

Theorem 7.1. For $x \in \mathbb{B}, \eta \in \mathbb{S}$, and a multi-index $\alpha$,

$$
\left|\left(\partial^{\alpha} R_{q}\right)(x, \eta)\right| \lesssim \begin{cases}1 & \text { if } q+|\alpha|<-n \\ 1+\log |x-\eta|^{-1} & \text { if } q+|\alpha|=-n \\ |x-\eta|^{-(n+q+|\alpha|)} & \text { if } q+|\alpha|>-n .\end{cases}
$$

Proof. Definition 3.1 says that if $q>-(1+n / 2)$, then

$$
R_{q}(x, \eta)=\frac{\Gamma(n / 2)}{\Gamma(1+n / 2+q)} \sum_{m=0}^{\infty} \frac{\Gamma(1+n / 2+q+m)}{\Gamma(n / 2+m)} Z_{m}(x, \eta)
$$

and if $q \leq-(1+n / 2)$, then

$R_{q}(x, \eta)=\Gamma\left(\frac{n}{2}\right) \Gamma\left(1-\left(\frac{n}{2}+q\right)\right) \sum_{m=0}^{\infty} \frac{\Gamma(1+m)}{\Gamma(1-(n / 2+q)+m)} \frac{\Gamma(1+m)}{\Gamma(n / 2+m)} Z_{m}(x, \eta)$. 
We apply either Lemma 7.3 or 7.4 ; in either case, $c=n+q+|\alpha|$; the result follows immediately.

This theorem generalizes to all $q \in \mathbb{R}$ what is obtained for the Bergman kernels in $[12$, Lemma 3.2(a)] (for $q=-1,0,1, \ldots$ ), [14, Lemma 2.7] (for $q \geq-1$ ), [32, Lemma 2.3] (for $q>0$ ), [29, Lemma 6(i); 33, Theorem 4.1; 6, Lemma 4.4; 31, Lemma 3.3] (for $q>-1$ ). Also related is [28, Lemma 3.1].

Using (2.4) and Theorem 7.1, we refine Proposition 3.2 as follows.

Corollary 7.1. For $x \in \mathbb{B}, y \in \overline{\mathbb{B}}$, and a multi-index $\alpha$,

$$
\left|\left(\partial^{\alpha} R_{q}\right)(x, y)\right| \lesssim \begin{cases}1 & \text { if } q+|\alpha|<-n \\ 2+\log \{x, y\}^{-1} & \text { if } q+|\alpha|=-n \\ \{x, y\}^{-(n+q+|\alpha|) / 2} & \text { if } q+|\alpha|>-n .\end{cases}
$$

For any $s, t \in \mathbb{R}$ with $t=|\alpha|$, exactly the same upper bounds hold for $D_{s}^{t} R_{q}$.

Proof. We just need to note that the homogeneous expansion of $D_{s}^{t} R_{q}$ has the same form as $R_{q+t}$ by Definition 3.2 continuing the idea in (3.11).

Pointwise upper bounds lead to integral upper bounds.

Proof of half of Theorem 1.5. This part on upper estimates is now straightforward. If $q>-n$, Corollary 7.1 gives

$$
\mathbb{I}_{q, a, b}(x) \lesssim \int_{\mathbb{B}} \frac{\left(1-|y|^{2}\right)^{b}}{\{x, y\}^{(n+q) a / 2}} d \nu(y) .
$$

Now Lemma 6.2 yields all three possibilities with respect to $c$.

If $q=-n$, then $c<-1$, and we need to show that $\mathbb{I}_{q, a, b}(x)$ is bounded. Now $\{x, y\}^{-1} \geq 1 / 4$, hence $2+\log \{x, y\}^{-1} \lesssim\{x, y\}^{-1 / 2 a}$. Then

$$
\mathbb{I}_{q, a, b}(x) \lesssim \int_{\mathbb{B}} \frac{\left(1-|y|^{2}\right)^{b}}{\{x, y\}^{1 / 2}} d \nu(y) .
$$

Lemma 6.2 gives us the desired boundedness.

There is nothing to prove for $q<-n$ by the work of Sec. 3, or by Corollary 7.1. Continuous extension to the boundary for $c<0$ follows by Remark 6.1.

For the Bergman kernel versions of this part of Theorem 1.5, see [12, Lemma 3.2] for $q \in \mathbb{N}$, [14, Lemma 2.10; 29, Proposition 8; 4, Corollary 1] for $a=1$, and all with $q>-1$ and $c>0$. One exception is [5, Lemma 3.5] in which $c \in \mathbb{R}$, but with $q=0$.

We next turn to the lower estimates on the Besov kernels. For them, Theorem 7.2 is crucial. But first we need to introduce some nontangential approach regions. Write $y=\left(y_{1}, y^{\prime \prime}\right)$ in which $y^{\prime \prime}=\left(y_{2}, \ldots, y_{n}\right)$; put also $y^{\prime}=y_{1} e_{1}$, where $e_{1}=(1,0, \ldots, 0)$. For $0<\lambda<1$, let

$$
U_{\lambda}=\left\{y \in \mathbb{B}: 0<y_{1}<1,\left|y^{\prime \prime}\right|<\lambda\left(1-y_{1}\right)\right\} .
$$


The regions $U_{\lambda}$ are cones in $\mathbb{B}$ with vertex at $e_{1}$ and aperture $\lambda$. Obviously, we can carry the $U_{\lambda}$ to cones with vertex at any $\xi \in \mathbb{S}$ by an orthogonal transformation of $\mathbb{R}^{n}$. If $y \in U_{\lambda}$, then $|y| \leq\left|y^{\prime}\right|+\left|y^{\prime \prime}\right| \leq\left|y^{\prime}\right|+\lambda\left(1-\left|y^{\prime}\right|\right)$. This and the more obvious reverse inequality show that $1-|y| \sim 1-\left|y^{\prime}\right|$.

Theorem 7.2. There exists a $\lambda<1 / 4$ such that for all $x=(r, 0, \ldots, 0) \in \mathbb{B}$ with $r>0$ and $y=\left(y_{1}, \ldots, y_{n}\right) \in U_{\lambda}$, we have

$$
\left|R_{q}(x, y)\right| \gtrsim \begin{cases}1 & \text { if } q<-n \\ 1+\log \left(1-|x|\left|y^{\prime}\right|\right)^{-1} & \text { if } q=-n \\ \left(1-|x|\left|y^{\prime}\right|\right)^{-(n+q)} & \text { if } q>-n\end{cases}
$$

where $y^{\prime}=\left(y_{1}, 0, \ldots, 0\right)$.

Proof. First, by the second claim in Proposition 3.2, for the particular $x$ and $y^{\prime}$, $R_{q}\left(x, y^{\prime}\right)$ is bounded below by an expression containing $r y_{1}$. The point of the lemma is that the same lower bound is valid also for $R_{q}(x, y)$ when $y \in U_{\lambda}$ for some suitable $\lambda$ with the same particular $x$. We pick $\lambda<1 / 4$ in all circumstances.

Let $y \in U_{\lambda}$; then $y=\left(y_{1}, y^{\prime \prime}\right) \in B_{e}\left(y^{\prime},\left(1-y_{1}\right) / 4\right)=: B_{1}$. By the mean value theorem of advanced calculus, there is a $z$ on the line segment joining $y$ and $y^{\prime}$ such that $R_{q}(x, y)=R_{q}\left(x, y^{\prime}\right)+\nabla_{z} R_{q}(x, z) \cdot\left(y-y^{\prime}\right)$. Hence

$$
\left|R_{q}(x, y)-R_{q}\left(x, y^{\prime}\right)\right| \leq \max _{z \in B_{1}}\left|\nabla_{z} R_{q}(x, z)\right|\left|y-y^{\prime}\right| .
$$

Note that if $z \in B_{1}$, then $B_{2}:=B_{e}\left(z,\left(1-y_{1}\right) / 4\right) \subset B_{e}\left(y^{\prime},\left(1-y_{1}\right) / 2\right):=B_{3}$. By the Cauchy estimates (see $[1,2.4]$ ), for all $z \in B_{1}$,

$$
\left|\nabla_{z} R_{q}(x, z)\right| \lesssim \frac{\max _{w \in B_{2}}\left|R_{q}(x, w)\right|}{\left(1-y_{1}\right) / 4} \lesssim \frac{\max _{w \in B_{3}}\left|R_{q}(x, w)\right|}{1-y_{1}} .
$$

A $y \in U_{\lambda}$ satisfies $\left|y-y^{\prime}\right|=\left|y^{\prime \prime}\right|<\lambda\left(1-y_{1}\right)$; hence

$$
\left|R_{q}(x, y)-R_{q}\left(x, y^{\prime}\right)\right| \lesssim \lambda \max _{w \in B_{3}}\left|R_{q}(x, w)\right| .
$$

If $w \in B_{3}$, then $|w|<\left|y^{\prime}\right|+\left(1-y_{1}\right) / 2=\left(1+y_{1}\right) / 2$ and $1-r|w|>\left(1-r y_{1}\right) / 2$.

If $q>-n$, then

$$
\left|R_{q}(x, w)\right| \lesssim \frac{1}{\left(1-r y_{1}\right)^{n+q}} \quad \text { and } \quad\left|R_{q}\left(x, y^{\prime}\right)-R_{q}(x, y)\right| \lesssim \frac{\lambda}{\left(1-r y_{1}\right)^{n+q}} .
$$

Thus for a small enough $\lambda$,

$$
\left|R_{q}(x, y)\right| \gtrsim \frac{1}{\left(1-r y_{1}\right)^{n+q}} \quad\left(y \in U_{\lambda}\right) .
$$

If $q=-n$, then

$$
\left|R_{q}(x, w)\right| \lesssim 1+\log \frac{1}{1-r y_{1}} ;
$$

and if $q<-n$, then $\left|R_{q}(x, w)\right|$ is bounded. In either case, the desired result is obtained similar to the previous case. 
For similar results on Bergman kernels, see [5, Lemma 3.4; 10, Lemma 2.3] for $q=0,[29$, Proposition 5; 6, Lemma 5.2] for $q>-1$.

Proof of other half of Theorem 1.5. We now prove the lower estimates. In the case $q>-n$, first let $x=(r, 0, \ldots, 0) \in \mathbb{B}$ with $r>0$. Theorem 7.2 provides us with a region $U_{\lambda}$ and a lower bound yielding

$$
\mathbb{I}_{q, a, b}(x) \gtrsim \int_{U_{\lambda}} \frac{\left(1-|y|^{2}\right)^{b}}{\left(1-r\left|y^{\prime}\right|\right)^{(n+q) a}} d \nu(y) \gtrsim \int_{0}^{1} \int_{\lambda\left(1-y_{1}\right) \mathbb{B}^{\prime}} \frac{\left(1-y_{1}^{2}\right)^{b} d \nu^{\prime}\left(y^{\prime \prime}\right)}{\left(1-r y_{1}\right)^{(n+q) a}} d y_{1},
$$

where $\mathbb{B}^{\prime}$ denotes the $(n-1)$-dimensional ball and $d \nu^{\prime}$ the normalized volume measure on it. Estimating the inner integral, we obtain

$$
\mathbb{I}_{q, a, b}(x) \gtrsim \int_{0}^{1} \frac{\left(1-y_{1}^{2}\right)^{n-1+b}}{\left(1-r^{2} y_{1}^{2}\right)^{(n+q) a}} d y_{1} .
$$

The result for the special $x$ now follows from Lemma 6.3. If $0 \neq x \in \mathbb{B}$ is arbitrary, we can find an orthogonal transformation $L$ of $\mathbb{R}^{n}$ that takes $x$ to $(|x|, 0, \ldots, 0)$. Using (14.6), a change of variables from $L y$ to $y$, and the invariance of $\nu$ under $L$, we are done.

In the case $q=-n$, we proceed as above using Theorem 7.2 and after one integration, we obtain

$$
\mathbb{I}_{q, a, b}(x) \gtrsim \int_{0}^{1}\left(1-y_{1}^{2}\right)^{n-1+b}\left(1+\log \frac{1}{1-r y_{1}}\right)^{a} d y_{1} .
$$

The logarithmic factor is $\geq 1$ and $n-1+b>0$, we see that $\mathbb{I}_{q, a, b}(x) \gtrsim 1$, and this is what we need to prove since $c<0$. In the case $q<-n$, again $c<0$ and we simply use $R_{q}(x, y) \gtrsim 1$ supplied by Theorem 7.2 .

For the Bergman kernel versions of this part of Theorem 1.5, see [5, Lemma 3.5] for $q=0$, [10, Lemma 2.4] for $q=b=0, a>1$, and $c>0$, and [29, Proposition 8] for $q>-1$ and $c>0$.

\section{Integral Operators on $L_{q}^{p}$}

In this section, we obtain the harmonic counterpart of the well-known result on the $L^{p}$-boundedness of certain integral operators with sesquiholomorphic kernels; see, for example, [44, Theorem 2.10].

What we really need are $T_{s, t}$ and $X_{s, t}$ introduced in Sec. $1 ; S_{s, t}$ is included, because we need an operator with a nonnegative kernel to be able to use the Schur test.

Lemma 8.1. If * represents the adjoint of an operator acting on $L_{q}^{p}, 1 \leq p<\infty$, with respect to the pairing induced by the inner product of $L_{q}^{2}$, then $T_{s, t}^{*}=T_{q+t,-q+s}$, $S_{s, t}^{*}=S_{q+t,-q+s}$, and $E_{s, t}^{*}=E_{q+t,-q+s}$, whenever the operators are bounded. 
Proof. Let $f \in L_{q}^{p}$ and $g \in L_{q}^{p^{\prime}}$. By definition, $\left[T_{s, t} f, g\right]_{L_{q}^{2}}=\left[f, T_{s, t}^{*} g\right]_{L_{q}^{2}}$. The adjoint appears naturally by writing down the definition in detail using integrals and using the real-valuedness of $R_{s+t}(x, y)$, positivity of $\{x, y\}$, and their symmetry in their two variables along with Fubini theorem.

Proof of Theorem 1.6. Notice that (c) implies $s+t>-1$ so that $T_{s, t}$ and $S_{s, t}$ are bounded only for the Bergman kernels $R_{s+t}$ given in (3.1).

(b) $\Rightarrow$ (a) and (d) $\Rightarrow$ (e) We clearly have $\left|T_{s, t} f(x)\right| \leq S_{s, t}(|f|)(x)$ and hence $\left\|T_{s, t}\right\| \leq\left\|S_{s, t}\right\|$; thus the boundedness of $S_{s, t}$ implies the boundedness of $T_{s, t}$. Similarly, the boundedness of $E_{s, t}$ implies the boundedness of $X_{s, t}$.

(a) $\Rightarrow$ (c) Let $f(y)=\left(1-\rho^{2}\right)^{c}$ with $c$ sufficiently large so that $q+p c>-1$ and $s+c>-1$. So $f \in L_{q}^{p}$ and $\nu_{s+c}$ is finite. Then the radiality of $d \nu_{s+c}$ combined with the mean value property and (3.4) yield

$$
\begin{aligned}
T_{s, t} f(x) & =V_{s+c}\left(1-r^{2}\right)^{t} \int_{\mathbb{B}} R_{s+t}(x, y) d \nu_{s+c}(y) \\
& =V_{s+c}\left(1-r^{2}\right)^{t} R_{s+t}(x, 0) \\
& =V_{s+c}\left(1-r^{2}\right)^{t},
\end{aligned}
$$

because $R_{s+t}$ is harmonic in either of its variables. By assumption $T_{s, t} f \in L_{q}^{p}$ and this implies $q+p t>-1$ which is the left inequality in (c).

For the other inequality in (c), first let $1<p<\infty$. The assumption of boundedness of $T_{s, t}$ on $L_{q}^{p}$ implies the boundedness of $T_{s, t}^{*}=T_{q+t,-q+s}$ on $L_{q}^{p^{\prime}}$. Applying the result of the previous paragraph, we obtain $q+p^{\prime}(-q+s)>-1$, that is, $q+1<p(s+1)$, which is the right inequality in (c).

If $p=1$, then $p^{\prime}=\infty$ and we let $f(y)=\left(1-\rho^{2}\right)^{c}$ with $c \geq 0$ and $q+t+c>-1$. So $f \in L^{\infty}$ and $\nu_{q+t+c}$ is finite. That $T_{s, t}^{*} f \in L^{\infty}$ implies $q \leq s$ as in the second paragraph of this proof. We now show that equality is not possible. If $q=s$, for fixed $x \in \mathbb{B}$, let $f_{x}(y)=\left|R_{s+t}(x, y)\right| / R_{s+t}(x, y)$ whenever the kernel is not 0 , and otherwise let $f_{x}(y)=1$. Then $\left\|f_{x}\right\|_{L^{\infty}}=1$ and

$$
\begin{aligned}
T_{s, t}^{*}\left(f_{x}\right)(x) & =\int_{\mathbb{B}}\left|R_{s+t}(x, y)\right|\left(1-\rho^{2}\right)^{s+t} d \nu(y) \\
& \sim \frac{1}{r^{2}} \log \frac{1}{1-r^{2}}
\end{aligned}
$$

since $q=s$ and by Theorem 1.5. Thus

$$
\begin{aligned}
\left\|T_{s, t}^{*}\left(f_{x}\right)\right\|_{L^{\infty}} & \gtrsim \frac{1}{r^{2}} \log \frac{1}{1-r^{2}} \quad \text { and } \\
\left\|T_{s, t}^{*}\right\| & \gtrsim \frac{1}{r^{2}} \log \frac{1}{1-r^{2}} .
\end{aligned}
$$

Letting $r \rightarrow 1^{-}$, we see that $T_{s, t}^{*}$ is unbounded contrary to assumption. Therefore we must have $q<s$. 
(c) $\Rightarrow$ (b) First let $p=1$ so that we have $q+t>-1, q<s$, and also $s+t>-1$. For $f \in L_{q}^{1}$, by Fubini theorem and Theorem 1.5, we have

$$
\begin{aligned}
\left\|S_{s, t} f\right\|_{L_{q}^{1}} & \leq \frac{1}{V_{q}} \int_{\mathbb{B}}|f(y)|\left(1-\rho^{2}\right)^{s} \int_{\mathbb{B}}\left|R_{s+t}(x, y)\right|\left(1-r^{2}\right)^{q+t} d \nu(x) d \nu(y) \\
& \sim \int_{\mathbb{B}}|f(y)|\left(1-\rho^{2}\right)^{s} \frac{1}{\left(1-\rho^{2}\right)^{s-q}} d \nu(y)=V_{q} \int_{\mathbb{B}}|f| d \nu_{q}<\infty .
\end{aligned}
$$

Thus $S_{s, t}$ is bounded on $L_{q}^{1}$.

Second, let $1<p<\infty$. We apply Theorem 6.1 with $\mu=\nu, \kappa=\nu_{q}$, and $h(y)=$ $\left(1-|y|^{2}\right)^{-(1+q) / p p^{\prime}}$. Clearly, $h$ is strictly positive on $\mathbb{B}$ and $\mu$ is absolutely continuous with respect to $\kappa$ with $(d \mu / d \kappa)(y)=V_{q}\left(1-|y|^{2}\right)^{-q}$. By (c) and Theorem 1.5, we have

$$
\begin{aligned}
S_{s, t}\left(h^{p^{\prime}}\right)(x) & =\left(1-r^{2}\right)^{t} \int_{\mathbb{B}}\left|R_{s+t}(x, y)\right|\left(1-\rho^{2}\right)^{s-(1+q) / p} d \nu(y) \\
& \sim\left(1-r^{2}\right)^{t} \frac{1}{\left(1-r^{2}\right)^{(1+q) / p+t}}=h(x)^{p^{\prime}} .
\end{aligned}
$$

By the symmetry of $R_{s+t}(x, y)$ in its variables, we check

$$
\begin{aligned}
S_{s, t}^{*}\left(h^{p}\right)(y) & =\left(1-\rho^{2}\right)^{-q+s} \int_{\mathbb{B}}\left|R_{s+t}(x, y)\right|\left(1-r^{2}\right)^{q+t-(1+q) / p^{\prime}} d \nu(x) \\
& \sim\left(1-\rho^{2}\right)^{-q+s} \frac{1}{\left(1-\rho^{2}\right)^{-q+s+(1+q) / p^{\prime}}}=h(y)^{p},
\end{aligned}
$$

which follows by (c) and Theorem 1.5. Therefore $S_{s, t}$ is bounded on $L_{q}^{p}$.

(d) $\Rightarrow$ (c) We consider $E_{s, t} f$ for $f(y)=\left(1-\rho^{2}\right)^{c}$ with $c$ sufficiently large so that $s+c>-1$ and $c>t$. Then by Lemma $6.2, E_{s, t} f(x) \sim\left(1-r^{2}\right)^{t}$. The assumption $E_{s, t} f \in L_{q}^{p}$ yields $q+p t>-1$. If $1<p<\infty$, the details of obtaining $q+1<p(s+1)$ from the boundedness of $E_{s, t}^{*}$ are exactly the same as for $T_{s, t}$, and so are the details of obtaining $q+1 \leq p(s+1)$ when $p=1$. To show equality is not possible, the function we pick is $f=1$ and the estimate we use is one in Lemma 6.2 again.

(c) $\Rightarrow$ (d) If $p=1$, we proceed as for $S_{s, t}$, but use an estimate in Lemma 6.2 instead. If $1<p<\infty$, we use Theorem 6.1 with the same function and measures as for $S_{s, t}$ in conjunction with Lemma 6.2 one more time. In either case we obtain (d), and complete the proof of the theorem.

We have not employed the full power of Theorem 1.5 in the proof above; taking $a=1$ sufficed. Using other values of $a$, we can obtain equivalent conditions for the boundedness of a large class of integral operators whose kernels carry powers of any of the Bergman-Besov reproducing kernels $R_{q}$. For example, [5, Proposition 3.6] does that by looking at powers of the one kernel $R_{0}$.

Operators that are identical or similar to $T_{s, t}$ and $S_{s, t}$ have been investigated before. See [12, Lemma 3.3; 28, Propositions 3.5 and 3.6; 15, Theorem 3.1; 32, Lemma 2.4]. They restrict themselves to the Bergman zone with assumptions like 
$s>0$ or $s+t>0$ from the outset, so none of them can take care of the Besov kernels.

\section{Independence of Order of Derivative}

We are now ready to relax the restriction on $t$ in (1.1) and replace it by the more natural (1.2). Our main goal is the proof of Theorem 1.1; then we pass on to other properties of the spaces $b_{q}^{p}$ that will benefit from a more freely selected $t$ in their definition. We start with some preparatory material.

For $m=0,1,2, \ldots$ and $a \in \mathbb{R}$, set $e_{m}:=e_{m}\left(a, s_{1}, s_{2}, t_{1}, t_{2}\right):=\gamma_{m}(a) \frac{d_{m}\left(s_{2}, t_{2}\right)}{d_{m}\left(s_{1}, t_{1}\right)}$ and define $\widetilde{K}(x, y):=\widetilde{K}_{a, s_{1}, s_{2}, t_{1}, t_{2}}(x, y):=\sum_{m=0}^{\infty} e_{m} Z_{m}(x, y)$ wherever the series converges. The parameter $a$ is yet to be chosen but should be considered large. By Definitions 3.2, 3.1, and (2.1), the coefficients grow as $e_{m} \sim m^{1+a+t_{2}-t_{1}}$, so in many respects, $\widetilde{K}$ is like $R_{a+t_{2}-t_{1}}$ and has the convergence properties described in Theorem 3.1. They also share properties such as (14.5) and (14.6) that are carried from the $Z_{m}$. We also have pointwise estimates much like Corollary 7.1.

Lemma 9.1. For $x, y \in \mathbb{B}$ and $n+a+t_{2}-t_{1}>0$,

$$
|\widetilde{K}(x, y)| \lesssim \frac{1}{\{x, y\}^{\left(n+a+t_{2}-t_{1}\right) / 2}} .
$$

Proof. Definitions 3.1 and 3.2 say that $\widetilde{K}(x, \eta)$ has the form of $W(x, \eta)$ of Lemma 7.4 with $c=n+a+t_{2}-t_{1}>0$. Then that lemma along with (2.4) yields the desired result.

Now we have everything to prove Theorem 1.1. The operator $X_{s, t}$ of Sec. 1 is developed for this purpose.

Proof of Theorem 1.1. Let $s_{1}, t_{1} \in \mathbb{R}$ and $s_{2}, t_{2} \in \mathbb{R}$ be two pairs of parameters satisfying (1.2) which places no restriction on $s_{1}, s_{2}$, and suppose $u \in b_{q}^{p}$ using the pair $s_{1}, t_{1}$ as in Definition 1.1 for example, that is, $I_{s_{1}}^{t_{1}} u \in L_{q}^{p}$. Let $a>q+p t_{1}>-1$; so $\nu_{a}$ is finite. Fix $x \in \mathbb{B}$ until the last paragraph of the proof. We first express $D_{s_{2}}^{t_{2}} u$ in terms of $D_{s_{1}}^{t_{1}} u$ with the help of the kernel $\widetilde{K}$. More precisely, we prove that

$$
D_{s_{2}}^{t_{2}} u(x)=\int_{\mathbb{B}} D_{s_{1}}^{t_{1}} u(y) \widetilde{K}(x, y) d \nu_{a}(y) \quad\left(u \in b_{q}^{p}, x \in \mathbb{B}\right) .
$$

Initially let $u \in \mathcal{H}_{m}$. Then $D_{s_{1}}^{t_{1}} u=d_{m}\left(s_{1}, t_{1}\right) u$ and

$$
\begin{aligned}
\int_{\mathbb{B}} D_{s_{1}}^{t_{1}} u(y) \widetilde{K}(x, y) d \nu_{a}(y) & =d_{m}\left(s_{1}, t_{1}\right) \sum_{l=0}^{\infty} \gamma_{l}(a) \frac{d_{l}\left(s_{2}, t_{2}\right)}{d_{l}\left(s_{1}, t_{1}\right)} \int_{\mathbb{B}} u(y) Z_{l}(x, y) d \nu_{a}(y) \\
& =\gamma_{m}(a) d_{m}\left(s_{2}, t_{2}\right) \int_{\mathbb{B}} u(y) Z_{m}(x, y) d \nu_{a}(y)
\end{aligned}
$$




$$
\begin{aligned}
& =\gamma_{m}(a) d_{m}\left(s_{2}, t_{2}\right) \frac{1}{\gamma_{m}(a)}\left[u(\cdot), Z_{m}(x, \cdot)\right]_{L^{2}(\sigma)} \\
& =d_{m}\left(s_{2}, t_{2}\right) u(x)=D_{s_{2}}^{t_{2}} u(x)
\end{aligned}
$$

using orthogonality, (5.4), Remark 5.1, and (14.4). The convergence of $\widetilde{K}(x, y)$ uniformly in $y \in \overline{\mathbb{B}}$ and the boundedness of $u$ justify the exchange of the order of integration and summation.

Suppose next $u \in b_{q}^{p} \cap h(\overline{\mathbb{B}})$ with the homogeneous expansion (14.9). Then

$$
\begin{aligned}
D_{s_{2}}^{t_{2}} u(x) & =\sum_{m=0}^{\infty} d_{m}\left(s_{2}, t_{2}\right) u_{m}(x) \\
& =\sum_{m=0}^{\infty} \int_{\mathbb{B}} d_{m}\left(s_{1}, t_{1}\right) u_{m}(y) \widetilde{K}(x, y) d \nu_{a}(y) \\
& =\int_{\mathbb{B}} \widetilde{K}(x, y) \sum_{m=0}^{\infty} d_{m}\left(s_{1}, t_{1}\right) u_{m}(y) d \nu_{a}(y) \\
& =\int_{\mathbb{B}} D_{s_{1}}^{t_{1}} u(y) \widetilde{K}(x, y) d \nu_{a}(y),
\end{aligned}
$$

in which the boundedness of $\widetilde{K}(x, y)$ in $y \in \mathbb{B}$ and Theorem 3.1 justify the exchange of the order of integration and summation.

Last, let $u \in b_{q}^{p}$ be arbitrary. Its dilates $u_{\tau}$ converge to $u$ in $b_{q}^{p}$ as $\tau \rightarrow 1^{-}$by Theorem 4.2; equivalently, $D_{s_{1}}^{t_{1}} u_{\tau} \rightarrow D_{s_{1}}^{t_{1}} u$ in $L_{q+p t_{1}}^{p}$. Then also $D_{s_{1}}^{t_{1}} u_{\tau} \rightarrow D_{s_{1}}^{t_{1}} u$ in $L_{q+p t_{1}}^{1}$ since $\nu_{q+p t_{1}}$ is finite. This also implies $D_{s_{1}}^{t_{1}} u_{\tau} \rightarrow D_{s_{1}}^{t_{1}} u$ in $L_{a}^{1}$ since we have chosen $a>q+p t_{1}$. In addition, uniformly on compact subsets of $\mathbb{B}, u_{\tau} \rightarrow u$ and hence $D_{s_{2}}^{t_{2}} u_{\tau} \rightarrow D_{s_{2}}^{t_{2}} u$ by Theorem 3.2. Now

$$
\begin{aligned}
\left|D_{s_{2}}^{t_{2}} u(x)-\int_{\mathbb{B}} D_{s_{1}}^{t_{1}} u(y) \widetilde{K}(x, y) d \nu_{a}(y)\right| \\
\leq\left|D_{s_{2}}^{t_{2}} u(x)-D_{s_{2}}^{t_{2}} u_{\tau}(x)\right| \\
+\left|D_{s_{2}}^{t_{2}} u_{\tau}(x)-\int_{\mathbb{B}} D_{s_{1}}^{t_{1}} u_{\tau}(y) \widetilde{K}(x, y) d \nu_{a}(y)\right| \\
+\left|\int_{\mathbb{B}} D_{s_{1}}^{t_{1}} u_{\tau}(y) \widetilde{K}(x, y) d \nu_{a}(y)-\int_{\mathbb{B}} D_{s_{1}}^{t_{1}} u(y) \tilde{K}(x, y) d \nu_{a}(y)\right| .
\end{aligned}
$$

The second term on the right is 0 by the previous paragraph. The first and third terms can be made small as $\tau \rightarrow 1^{-}$by the explanations above; in the third we also use that $\widetilde{K}(x, y)$ is bounded in $y \in \mathbb{B}$. Thus (9.1) is proved for all $u \in b_{q}^{p}$.

What equation (9.1) in conjunction with Lemma 9.1 accomplishes is the fact that $\left(1-|x|^{2}\right)^{t_{2}-t_{1}} D_{s_{2}}^{t_{2}} u(x)=X_{a, t_{2}-t_{1}}\left(D_{s_{1}}^{t_{1}} u\right)(x)$ for $x \in \mathbb{B}$. By (1.2), if $a$ is sufficiently large, the inequalities $-p\left(t_{2}-t_{1}\right)<q+p t_{1}+1<p(a+1)$ obtain. Then Theorem 1.6 implies that $X_{a, t_{2}-t_{1}}$ maps $L_{q+p t_{1}}^{p}$ to itself boundedly. Since $D_{s_{1}}^{t_{1}} u \in L_{q+p t_{1}}^{p}$ by our 
initial choice, this means that $\left(1-|x|^{2}\right)^{t_{2}-t_{1}} D_{s_{2}}^{t_{2}} u(x) \in L_{q+p t_{1}}^{p}$ as well; equivalently, $I_{s_{2}}^{t_{2}} u \in L_{q}^{p}$. Written in a different way, $\left\|I_{s_{2}}^{t_{2}} u\right\|_{L_{q}^{p}} \lesssim\left\|I_{s_{1}}^{t_{1}} u\right\|_{L_{q}^{p}}$, and we have the reverse inequality by exchanging the roles of the indices 1 and 2 . This gives the claimed equivalence of the norms.

The holomorphic counterparts of Theorem 1.1 are [2, Theorem 5.12(i); 17, Theorem $4.1(\mathrm{a})]$.

Therefore each selection of $t$ satisfying (1.2) and of $s$ gives rise to an equivalent norm on $b_{q}^{p}$. We keep calling all these norms $\|u\|_{b_{q}^{p}}$; they have the form

$$
\|u\|_{b_{q}^{p}}^{p}=\left\|I_{s}^{t} u\right\|_{L_{q}^{p}}^{p}=\frac{1}{V_{q}} \int_{\mathbb{B}}\left|D_{s}^{t} u(x)\right|^{p}\left(1-|x|^{2}\right)^{q+p t} d \nu(x) .
$$

Let us see what we can deduce with this freedom in choosing parameters.

Corollary 9.1. If $q_{1}<q_{2}$, then $b_{q_{1}}^{p} \subset b_{q_{2}}^{p}$, and the associated inclusion map $i$ is bounded with norm at most $V_{q_{1}} / V_{q_{2}}$ with suitable $s, t$ used in norms.

Proof. Pick a $t$ satisfying (1.2) with $q=q_{1}$; then it satisfies (1.2) with $q=q_{2}$ too. The result follows from Theorem 1.1 since $L_{q_{1}}^{p} \subset L_{q_{2}}^{p}$. The claims on $i$ are clear by writing the norms explicitly as in (9.2).

Corollary 9.2. Given $q, p$, for any $s, t \in \mathbb{R}, D_{s}^{t}: b_{q}^{p} \rightarrow b_{q+p t}^{p}$ is an isomorphism, and $\frac{V_{q+p t}}{V_{q}} D_{s}^{t}$ is an isometry when norms with appropriate parameters $s, t$ are used in the two spaces.

Proof. Let $u \in h(\mathbb{B})$ and put $v=D_{s}^{t} u$, which is also in $h(\mathbb{B})$. Take $t_{1}$ so large that $q+p\left(t+t_{1}\right)>-1$. Then $D_{s+t}^{t_{1}} v=D_{s+t}^{t_{1}} D_{s}^{t} u=D_{s}^{t+t_{1}} u$ by $(3.10)$. If $u \in b_{q}^{p}$, then $I_{s}^{t+t_{1}} u \in L_{q}^{p}$, and this is equivalent to $I_{s+t}^{t_{1}} v \in L_{q+p t}^{p}$ which means $v \in b_{q+p t}^{p}$ by Theorem 1.1. Conversely, if $v \in b_{q+p t}^{p}$, then $I_{s+t}^{t_{1}} v \in L_{q+p t}^{p}$, and this is equivalent to $I_{s}^{t+t_{1}} u \in L_{q}^{p}$ which means $u \in b_{q}^{p}$ by Theorem 1.1 again. Together with (3.9), the isomorphism claim follows. The equality of the two norms $\|u\|_{b_{q}^{p}}=\left\|I_{s}^{t+t_{1}} u\right\|_{L_{q}^{p}}$ and $\left\|\frac{V_{q+p t}}{V_{q}} v\right\|_{b_{q+p t}^{p}}=\left\|\frac{V_{q+p t}}{V_{q}} I_{s+t}^{t_{1}} v\right\|_{L_{q+p t}^{p}}$ is obvious from the above, and this proves the claim on isometry.

\section{Real Hyperbolic Metric and Atomic Decomposition}

Similar to those on the unit disk, for any $n$ and each $y \in \mathbb{B}$, there is the transformation

$$
\varphi_{y}(x)=\frac{|x-y|^{2} y+\left(1-|y|^{2}\right)(y-x)}{\{x, y\}} \quad(x \in \mathbb{B})
$$

which is bijective from $\mathbb{B}$ to $\mathbb{B}$. It is involutive, that is, its inverse is itself on $\mathbb{B}$, exchanges 0 and $y$, and has the most useful property

$$
1-\left|\varphi_{y}(x)\right|^{2}=\frac{\left(1-|x|^{2}\right)\left(1-|y|^{2}\right)}{\{x, y\}} \quad(x, y \in \mathbb{B}) .
$$


The Jacobian of $\varphi_{y}$ has the values

$$
\left(J \varphi_{y}\right)(x)=(-1)^{n} \frac{\left(1-|y|^{2}\right)^{n}}{\{x, y\}^{n}} \quad \text { and } \quad\left(J \varphi_{y}\right)\left(\varphi_{y}(x)\right)=\frac{1}{\left(J \varphi_{y}\right)(x)} .
$$

A Möbius transformation is defined as a composition of a $\varphi_{y}$ with an orthogonal transformation of $\mathbb{R}^{n}$ (an $n \times n$ orthogonal matrix).

We prove an invariance property of the measures $\nu_{q}$. It appears in $[40$, Theorem 3.4] for $q=-n$ and in [11, Proposition 3.1] for $q>-1$.

Proposition 10.1. If $\psi$ is a Möbius transformation and $f \in L_{q}^{1}$, then

$$
\int_{\mathbb{B}}(f \circ \psi)|J \psi|^{1+q / n} d \nu_{q}=\int_{\mathbb{B}} f d \nu_{q} .
$$

In other words,

$$
d \nu_{q}(x)=|J \psi(x)|^{-1-q / n} d \nu_{q}(\psi(x)) .
$$

In particular, $\nu_{-n}$ is Möbius-invariant on $\mathbb{B}$.

Proof. If $\psi=L$ is an orthogonal transformation of $\mathbb{R}^{n}$, then $J L= \pm 1$, and the proposition states the obvious fact that the radial measures $\nu_{q}$ are invariant under such $L$. So now let $\psi=\varphi_{y}$. Putting $g=\left(f \circ \varphi_{y}\right)\left|J \varphi_{y}\right|^{1+q / n}$, we compute by changing variables from $\varphi_{y}(x)$ to $x$, and using (10.2) and (10.1):

$$
\begin{aligned}
\int_{\mathbb{B}} g d \nu_{q} & =\frac{1}{V_{q}} \int_{\mathbb{B}} f(x)\left|\left(J \varphi_{y}\left(\varphi_{y}(x)\right)\right)^{1+q / n}\right|\left(1-\left|\varphi_{y}(x)\right|^{2}\right)^{q}\left|\left(J \varphi_{y}\right)(x)\right| d \nu(x) \\
& =\frac{1}{V_{q}} \int_{\mathbb{B}} f(x) \frac{1}{\left|\left(J \varphi_{y}(x)\right)^{q / n}\right|} \frac{\left(1-|x|^{2}\right)^{q}\left(1-|y|^{2}\right)^{q}}{\{x, y\}^{q}} d \nu(x) \\
& =\frac{1}{V_{q}} \int_{\mathbb{B}} f(x)\left(1-|x|^{2}\right)^{q} d \nu(x) \\
& =\int_{\mathbb{B}} f d \nu_{q} .
\end{aligned}
$$

Corollary 10.1. Let $\psi$ be a Möbius transformation. Then

$$
O_{\psi} u=(u \circ \psi)|J \psi|^{1 / p+q / n p}
$$

is a linear surjective isometry from $L_{q}^{p}$ to $L_{q}^{p}$. In particular, if $p=2$, then $O_{\psi}$ is an unitary transformation from $L_{q}^{2}$ to $L_{q}^{2}$.

Proof. Linearity is obvious. The isometry claim is an immediate consequence of Proposition 10.1. Surjectivity follows from $O_{\psi}^{-1}=O_{\psi^{-1}}$ by $(10.2)$.

The real hyperbolic metric is

$$
d(x, y)=\frac{1}{2} \log \frac{1+\left|\varphi_{y}(x)\right|}{1-\left|\varphi_{y}(x)\right|}=\tanh ^{-1} \frac{|x-y|}{\sqrt{\{x, y\}}} \quad(x, y \in \mathbb{B}) .
$$


A hyperbolic ball $B(z, \rho)$, that is, a ball in $d$ with hyperbolic center $z \in \mathbb{B}$ and hyperbolic radius $\rho>0$, is also a Euclidean ball $B_{e}\left(z_{e}, \rho_{e}\right)$ (not an ellipsoid) with $z_{e} \in \mathbb{B}$ and $0<\rho_{e}<1$, where

$$
z_{e}=\frac{\left(1-\tanh ^{2} \rho\right) z}{1-|z|^{2} \tanh ^{2} \rho} \text { and } \rho_{e}=\frac{\left(1-|z|^{2}\right) \tanh \rho}{1-|z|^{2} \tanh ^{2} \rho} .
$$

So $z$ and $z_{e}$ are in the same direction and $|z|>\left|z_{e}\right|$; also $\tanh \rho>\rho_{e}$. As is usual, $\varphi_{y}(B(z, \rho))=B\left(\varphi_{y}(z), \rho\right)$.

A more sophisticated application of Theorem 1.1 is to the atomic decomposition of functions in Besov spaces. These are developed in [9] for the unweighted Bergman spaces, and we boost their formulas to all real $q$. For another approach, see [12, Theorem 3].

For $\lambda>0$, a $\lambda$-lattice in $\mathbb{B}$ is a countable set $\left\{y_{j}\right\}$ of points in $\mathbb{B}$ such that the hyperbolic balls $B\left(y_{j}, \lambda\right)$ cover $\mathbb{B}$ and $d\left(y_{j}, y_{l}\right) \geq \lambda / a$ for some large enough $a>1$ if $j \neq l$.

Theorem 10.1. Given $q$ and $p$, let $s$ satisfy (1.3). There is a $\lambda_{0}>0$ such that if $\left\{y_{j}\right\}$ is a $\lambda$-lattice in $\mathbb{B}$ with $\lambda<\lambda_{0}$ and $u \in b_{q}^{p}$, then there are $c_{j} \in \mathbb{C}$ so that

$$
u(x)=\sum_{j=1}^{\infty} c_{j} R_{s}\left(x, y_{j}\right)\left(1-\left|y_{j}\right|^{2}\right)^{s-q / p+n / p^{\prime}} \quad(x \in \mathbb{B}),
$$

where the series converges absolutely and uniformly on compact subsets of $\mathbb{B}$ and in $\|\cdot\|_{b_{q}^{p}}$, and the $\ell^{p}$ norm of $\left\{c_{j}\right\}$ is equivalent to the $b_{q}^{p}$ norm of $u$.

Proof. If $u \in b_{q}^{p}$, then $v=D_{s}^{-q / p} u \in b_{0}^{p}$ for any $s$. Pick $s$ so that the condition $p(1+\alpha)>1$ of $[9$, Theorem 1] is satisfied with $\alpha=s-q / p$. It is interesting that this condition is the same as (1.3). Using [9, Theorem 1], we obtain the $\lambda$-lattice $\left\{y_{j}\right\}$ and the sequence $\left\{c_{j}\right\}$ with which the formula

$$
v(x)=\sum_{j=1}^{\infty} c_{j} R_{s-q / p}\left(x, y_{j}\right)\left(1-\left|y_{j}\right|^{2}\right)^{s-q / p+n / p^{\prime}} \quad(x \in \mathbb{B})
$$

holds. This series converges in $\|\cdot\|_{b_{0}^{p}}$ and absolutely and uniformly on compact subsets of $\mathbb{B}$, and also $\|v\|_{b_{0}^{p}} \lesssim\left\|\left\{c_{j}\right\}\right\|_{\ell^{p}}$ by [9, Proposition 2] and its proof. The reverse inequality $\left\|\left\{c_{j}\right\}\right\|_{\ell^{p}} \lesssim\|v\|_{b_{0}^{p}}$ is obtained from the boundedness of the operator $T_{p}$ and the invertibility of the operator $Q_{\alpha, p} T_{p}$ used in the proof of $[9$, Theorem 1]. By (3.11), we have $D_{s-q / p}^{q / p} R_{s-q / p}(x, \cdot)=R_{s}(x, \cdot)$. Corollary 9.2 yields $u=D_{s-q / p}^{q / p} v \in b_{q}^{p}$ and all the $u \in b_{q}^{p}$ are obtained this way. Applying $D_{s-q / p}^{q / p}$ to the series for $v$, which is a homogeneous expansion in $x$, we obtain the desired series expansion for $u$, which converges absolutely and uniformly on compact subsets of $\mathbb{B}$ by Theorem 3.2. The equivalences $\|u\|_{b_{q}^{p}} \sim\left\|D_{s}^{-q / p} u\right\|_{b_{0}^{p}}=\|v\|_{b_{0}^{p}} \sim\left\|\left\{c_{j}\right\}\right\|_{\ell^{p}}$ follow from Theorem 1.1. They also prove the convergence in $\|\cdot\|_{b_{q}^{p}}$ of the series for $u$. 


\section{Bergman-Besov Projections}

We prove Theorem 1.4 in this section and a few immediate consequences. With the hard work on the integral estimates of the Besov kernels in Sec. 7 and the ensuing theorem on integral operators in Sec. 8, our job is easy.

Proof of Theorem 1.4. Let $f \in L_{q}^{p}$ and $x \in \mathbb{B}$, and suppose (1.3) holds. Such an $f$ belongs to $L_{s}^{1}$. This is a simple application of Hölder inequality for $p>1$ :

$$
\begin{aligned}
\int_{\mathbb{B}}|f| d \nu_{s} & =\frac{1}{V_{s}} \int_{\mathbb{B}}|f(y)|\left(1-|y|^{2}\right)^{q / p}\left(1-|y|^{2}\right)^{s-q / p} d \nu(y) \\
& \lesssim\|f\|_{L_{q}^{p}}\left(\int_{\mathbb{B}}\left(1-|y|^{2}\right)^{(s-q / p) p^{\prime}} d \nu(y)\right)^{1 / p^{\prime}}<\infty
\end{aligned}
$$

because (1.3) implies $(s-q / p) p^{\prime}>-1$. If $p=1$, then $s>q$ by (1.3), and hence $f \in L_{q}^{1} \subset L_{s}^{1}$.

We first prove that $Q_{s} f$ is harmonic in $\mathbb{B}$. By Proposition 3.1, the series $R_{s}$ converges uniformly in $y \in \mathbb{B}$ for a given $x \in \mathbb{B}$. Then

$$
\begin{aligned}
Q_{s} f(x) & =\int_{\mathbb{B}} f(y) \sum_{m=0}^{\infty} \gamma_{m}(s) Z_{m}(x, y) d \nu_{s}(y) \\
& =\sum_{m=0}^{\infty} \gamma_{m}(s) \int_{\mathbb{B}} f(y) Z_{m}(x, y) d \nu_{s}(y)=: \sum_{m=0}^{\infty} \gamma_{m}(s) f_{m}(x),
\end{aligned}
$$

where the $f_{m}$ are defined by the last integral. Being a polynomial, each $Z_{m}$ is bounded on $\mathbb{B} \times \mathbb{B}$. Hence $\left|f_{m}(x)\right| \lesssim\|f\|_{L_{s}^{1}}$ and the $f_{m}$ are well-defined on $\mathbb{B}$. By differentiating under the integral sign, we see that the $f_{m}$ are harmonic functions of $x \in \mathbb{B}$ since the $Z_{m}$ are.

Now let $K \subset \mathbb{B}$ be compact and $x \in K$; then $r \leq r_{0}$ for some $r_{0}<1$. By the homogeneity of $Z_{m}$ and (14.7), we have $\left|f_{m}(x)\right| \leq r_{0}^{m} \delta_{m}\|f\|_{L_{s}^{1}} \lesssim \delta_{m} r_{0}^{m}$ for all $x \in K$. Then

$$
\sum_{m=0}^{\infty}\left|\gamma_{m}(s) f_{m}(x)\right| \leq \sum_{m=0}^{\infty} \gamma_{m}(s) \delta_{m} r_{0}^{m} \sim 1+\sum_{m=1}^{\infty} m^{n-1+s} r_{0}^{m}<\infty \quad(x \in K)
$$

much like (3.6), giving the uniform convergence of the left-hand side on compact subsets of $\mathbb{B}$. This implies that $Q_{s} f \in h(\mathbb{B})$.

The harmonic function $Q_{s} f$ belongs to $b_{q}^{p}$ if and only if $I_{s}^{t} Q_{s} f \in L_{q}^{p}$ for some $t$ satisfying (1.2). We show this membership for any such $t$. Each $f_{m}(x)$ above is homogeneous of degree $m$ since each $Z_{m}(x, \cdot)$ is, and the series on the right-hand side of (11.1) is the homogeneous expansion of $Q_{s} f$. Then

$$
D_{s}^{t} Q_{s} f=D_{s}^{t} \sum_{m=0}^{\infty} \gamma_{m}(s) f_{m}=\sum_{m=0}^{\infty} \gamma_{m}(s+t) f_{m}=\int_{\mathbb{B}} f(y) R_{s+t}(x, y) d \nu_{s}(y)
$$


by Definition 3.2 and (11.1) in reverse. This shows that

$$
I_{s}^{t} Q_{s} f=\frac{1}{V_{s}} T_{s, t} f .
$$

What we have just done is equivalent to differentiating $Q_{s} f$ under the integral sign and using (3.11). We know that $T_{s, t}$ is bounded on $L_{q}^{p}$ if and only if the inequalities in Theorem 1.6(c) are fulfilled, which are nothing but (1.2) and (1.3). This proves the statement about the boundedness of the $Q_{s}$.

Finally let $u \in b_{q}^{p}$ be given by its homogeneous expansion $u=\sum_{m=0}^{\infty} u_{m}$, let $x \in \mathbb{B}$, and $s$ and $t$ satisfy (1.3) and (1.2) so that $s+t>-1$. We prove that any such $I_{s}^{t}$ is a right inverse for $Q_{s}$. We have

$$
\begin{aligned}
Q_{s} I_{s}^{t} u(x) & =\frac{1}{V_{s}} \int_{\mathbb{B}} D_{s}^{t} u(y) R_{s}(x, y)\left(1-|y|^{2}\right)^{s+t} d \nu(y) \\
& =\frac{1}{V_{s}} \int_{\mathbb{B}} D_{s}^{t} u(y) \sum_{m=0}^{\infty} \gamma_{m}(s) Z_{m}(x, y)\left(1-|y|^{2}\right)^{s+t} d \nu(y) \\
& =\frac{1}{V_{s}} \sum_{m=0}^{\infty} \gamma_{m}(s) \int_{\mathbb{B}} D_{s}^{t} u(y) Z_{m}(x, y)\left(1-|y|^{2}\right)^{s+t} d \nu(y) \\
& =: \frac{1}{V_{s}} \sum_{m=0}^{\infty} \gamma_{m}(s) M_{s, t, m}(x),
\end{aligned}
$$

where the exchange of the order of summation and integration is justified by the uniform convergence of the series defining $R_{s}$ in $y \in \mathbb{B}$ for fixed $x$. By Corollary 9.2, $D_{s}^{t} u \in b_{q+p t}^{p}$. A replica of the computation above giving $f \in L_{s}^{1}$ shows that $D_{s}^{t} u \in$ $L_{s+t}^{1}$ as well. Combined with the boundedness of $Z_{m}$ on $\mathbb{B} \times \mathbb{B}$, this yields that $M_{s, t, m}(x)$ is finite for each $x$. Then by orthogonality, (5.4), Remark 5.1, (14.4), and Definition 3.2 , we obtain

$$
\begin{aligned}
M_{s, t, m}(x) & =d_{m}(s, t) \frac{V_{s+t}}{\gamma_{m}(s+t)}\left[u_{m}(\cdot), Z_{m}(x, \cdot)\right]_{L^{2}(\sigma)} \\
& =\frac{V_{s+t}}{\gamma_{m}(s)} u_{m}(x)
\end{aligned}
$$

where the exchange of the order of integration and summation is justified by the uniform convergence of the homogeneous expansion of $u$ on the compact subset $\rho \mathbb{S}$. Therefore

$$
Q_{s} I_{s}^{t} u(x)=\frac{1}{V_{s}} \sum_{m=0}^{\infty} \gamma_{m}(s) \frac{V_{s+t}}{\gamma_{m}(s)} u_{m}(x)=\frac{V_{s+t}}{V_{s}} u(x) .
$$

This completes the proof.

Remark 11.1. Thus all the harmonic Besov spaces $b_{q}^{p}$ can be realized as quotient spaces of weighted Lebesgue spaces. 
Corollary 11.1. If (1.2) and (1.3) hold, then we have the following operator identities:

(a) $Q_{s} I_{s}^{t}=\frac{V_{s+t}}{V_{s}} I$ on $b_{q}^{p}$.

(b) $I_{s}^{t} Q_{s}=\frac{1}{V_{s}} T_{s, t}$ on $L_{q}^{p}$.

(c) $T_{s, t} I_{s}^{t}=V_{s+t} I_{s}^{t}$ on $b_{q}^{p}$.

(d) $Q_{s} T_{s, t}=V_{s+t} Q_{s}$ on $L_{q}^{p}$.

Part (a) for $s=0$ with $t=1$ is in [41, Theorem 4.1]. A version of part (a) for $p>1, s=0$, and nonnegative integer $t$ but for harmonic functions on a bounded smooth domain appears in [25, Proposition 2]. See also [8, Theorem 1.1] for similar formulas for a different kind of projection.

Proof. Part (a) is (1.4) repeated. We have noted (b) in the proof of Theorem 1.4. And (c) and (d) follow immediately from these.

Remark 11.2. The map $Q_{s}: L_{q}^{p} \rightarrow b_{q}^{p}$ is in general not a true projection onto a subspace, because $b_{q}^{p}$ is not defined as a subspace of $L_{q}^{p}$, but rather by imbedding it in $L_{q}^{p}$ via $I_{s}^{t}$. However, since $b_{q}^{p}$ is complete, the imbedded spaces $I_{s}^{t}\left(b_{q}^{p}\right)$ is a closed subspace of $L_{q}^{p}$. Then Corollary 11.1 shows that the map $\frac{V_{s}}{V_{s+t}} I_{s}^{t} Q_{s}$ is a true projection from $L_{q}^{p}$ onto its subspace $I_{s}^{t}\left(b_{q}^{p}\right)$. When $q>-1$ and $p \geq 1$, which is part of the Bergman zone, we can take $t=0$ and $s=q$, the imbedding is via inclusion, and Corollary 11.1(a) says $Q_{s}=I$ on $b_{q}^{p}$ as well-known.

Bergman projections on harmonic spaces have been studied for some time. See [41, Theorem 3.1] for $s=q=0$ and $p>1$, [14, Theorem 3.1] for $s=q>-1$ and $p>1$, [32, Theorem 2.5] for $s>0, q>-1$, and $p>1$, [4, Theorem 2] for $s>0$ and $p>1$ on Bergman spaces with different weights, and [8, Theorem 3.1] for a different kind of projection. Of course all these sources deal solely with the Bergman zone on $\mathbb{B}$. The only result we know of on Besov spaces is $[15$, Theorem 4.1] in which $s>-1$ and $q=-n$. Recall that in Theorem 1.4 we have $q, s \in \mathbb{R}$ unrestrictedly.

Corollary 11.2. When they are bounded, $Q_{s}: L_{q}^{p} \rightarrow b_{q}^{p}$ and $I_{s}^{t}: b_{q}^{p} \rightarrow L_{q}^{p}$ are not compact.

Proof. The compactness of one of them would imply that the identity map on $b_{q}^{p}$ is compact by Corollary 11.1, because the product of a bounded map and a compact map is compact. But the identity map on an infinite-dimensional space is never compact.

\section{Partial Derivatives}

In this section, we prove the equivalence of defining the harmonic Besov spaces using either partial derivatives $\partial^{\alpha}$, usual radial derivatives $\mathcal{R}^{l}$, or radial differential operators $D_{s}^{t}$. Our tools are Theorem 1.4 on projections and integral representations 
(1.5), integral operators of Sec. 8, the pointwise estimates in Sec. 7 on the kernels, and more estimates. Our presentation is influenced by the methods of [44, Sec. 2.3], but the technical details are quite different and the heavy work of previous sections is essential. We start with Bergman spaces and derivatives of order 1 and build up from there.

Lemma 12.1. For $q>-1$ and $u \in h(\mathbb{B})$, the following are equivalent:

(a) $u \in b_{q}^{p}$.

(b) $\left(1-|x|^{2}\right)\left(\partial_{i} u\right)(x) \in L_{q}^{p}$, that is, $\partial_{i} u \in b_{q+p}^{p}$, for each $i=1, \ldots, n$.

(c) $\left(1-|x|^{2}\right)(\mathcal{R} u)(x) \in L_{q}^{p}$, that is, $\mathcal{R} u \in b_{q+p}^{p}$.

This result appears in [11, Theorem 3.7] with a different proof. Our proof is a nice application of Bergman-Besov projections.

Proof. (a) $\Rightarrow$ (b) Let $s$ satisfy (1.3); so $s>-1$ and $n+s+1>0$. For $t=0$ and $u \in b_{q}^{p}$, Theorem 1.4 in the guise of (1.5) implies

$$
u(x)=\int_{\mathbb{B}} u(y) R_{s}(x, y) d \nu_{s}(y) .
$$

We take $\partial_{i}$ and differentiate $R_{s}$ in the first variable under the integral sign, and then use the estimate in Corollary 7.1 to have

$$
\begin{gathered}
\partial_{i} u(x)=\int_{\mathbb{B}} u(y) \partial_{i} R_{s}(x, y) d \nu_{s}(y), \\
\left(1-|x|^{2}\right)\left|\partial_{i} u(x)\right| \lesssim\left(1-|x|^{2}\right) \int_{\mathbb{B}}|u(y)| \frac{1}{\{x, y\}^{(n+s+1) / 2}}\left(1-|y|^{2}\right)^{s} d \nu(y) .
\end{gathered}
$$

Now Theorem 1.6 with $t=1$ implies that $\left(1-|x|^{2}\right)\left(\partial_{i} u\right)(x) \in L_{q}^{p}$ since $|u| \in L_{q}^{p}$ by hypothesis.

(b) $\Rightarrow$ (c) This is obvious by (2.6).

(c) $\Rightarrow$ (a) Let $s$ satisfy (1.3) with $q+p$ replacing $q$; so $q+1<p s, s>0$ and $n+s-1>0$. Since $q+p>-1$, with $t=0$ and $\mathcal{R} u \in b_{q+p}^{p}$, again (1.5) implies

$$
\mathcal{R} u(x)=\int_{\mathbb{B}} \mathcal{R} u(y) R_{s}(x, y) d \nu_{s}(y) .
$$

Setting $x=0$ and using (2.6) and (3.4) give $\int_{\mathbb{B}} \mathcal{R} u(y) d \nu_{s}(y)=0$. Subtracting this from the previous equation yields

$$
\mathcal{R} u(x)=\int_{\mathbb{B}} \mathcal{R} u(y)\left(R_{s}(x, y)-1\right) d \nu_{s}(y) .
$$

Then we replace $x$ by $\tau x$, divide by $\tau$, use (2.7) and the Fubini theorem to obtain

$$
\begin{aligned}
u(x)-u(0) & =\int_{0}^{1} \int_{\mathbb{B}} \mathcal{R} u(y)\left(R_{s}(\tau x, y)-1\right) d \nu_{s}(y) \frac{d \tau}{\tau} \\
& =\int_{\mathbb{B}} \mathcal{R} u(y) \int_{0}^{1} \frac{R_{s}(\tau x, y)-1}{\tau} d \tau d \nu_{s}(y) .
\end{aligned}
$$


By $(3.3),\left(R_{s}(\tau x, y)-1\right) / \tau=\sum_{m=1}^{\infty} \gamma_{m}(q) \tau^{m-1} Z_{m}(x, y)$, and for $0 \leq \tau \leq 1 / 2$, this series converges uniformly in $x, y, \tau$ as shown in detail in (3.6), and hence is uniformly bounded. On the other hand, for $1 / 2<\tau \leq 1$, Corollary 7.1 shows $\left|R_{s}(\tau x, y)-1\right| / \tau \leq 2\left(\left|R_{s}(\tau x, y)\right|+1\right) \lesssim\{\tau x, y\}^{-(n+s) / 2}$. Thus by (2.4) and Lemma 6.1, we see that

$$
\begin{aligned}
|u(x)-u(0)| & \leq \int_{\mathbb{B}}|\mathcal{R} u(y)| \int_{0}^{1}\left|\frac{R_{s}(\tau x, y)-1}{\tau}\right| d \tau d \nu_{s}(y) \\
& \lesssim \int_{\mathbb{B}}|\mathcal{R} u(y)| \int_{0}^{1} \frac{d \tau}{\{\tau \rho x, \eta\}^{(n+s) / 2}} d \nu_{s}(y) \\
& \lesssim \int_{\mathbb{B}}|\mathcal{R} u(y)| \frac{1}{\{x, y\}^{(n+s-1) / 2}} d \nu_{s}(y) \\
& \lesssim \int_{\mathbb{B}}|\mathcal{R} u(y)|\left(1-|y|^{2}\right) \frac{1}{\{x, y\}^{(n+s-1) / 2}}\left(1-|y|^{2}\right)^{s-1} d \nu(y) .
\end{aligned}
$$

Now Theorem 1.6 with $t=0$ implies that $|u(x)-u(0)| \in L_{q}^{p}$ since by hypothesis $|\mathcal{R} u(y)|\left(1-|y|^{2}\right) \in L_{q}^{p}$. Because $u$ is harmonic, we conclude that $u \in b_{q}^{p}$.

We next move to higher-order derivatives, but still in Bergman spaces.

Lemma 12.2. For $q>-1$ and $u \in h(\mathbb{B})$, the following are equivalent:

(a) $u \in b_{q}^{p}$.

(b) For every $l \in \mathbb{N}$ and for every multi-index $\alpha$ with $|\alpha|=l$, it is satisfied that $\left(1-|x|^{2}\right)^{l}\left(\partial^{\alpha} u\right)(x) \in L_{q}^{p}$, that is, $\partial^{\alpha} u \in b_{q+p l}^{p}$.

(c) There is an $l \in \mathbb{N}$ such that for every multi-index $\alpha$ with $|\alpha|=l$, it holds that $\left(1-|x|^{2}\right)^{l}\left(\partial^{\alpha} u\right)(x) \in L_{q}^{p}$, that is, $\partial^{\alpha} u \in b_{q+p l}^{p}$.

(d) For every $l \in \mathbb{N}$, we have $\left(1-|x|^{2}\right)^{l}\left(\mathcal{R}^{l} u\right)(x) \in L_{q}^{p}$, that is, $\mathcal{R}^{l} u \in b_{q+p l}^{p}$.

(e) There is an $l \in \mathbb{N}$ such that $\left(1-|x|^{2}\right)^{l}\left(\mathcal{R}^{l} u\right)(x) \in L_{q}^{p}$, that is, $\mathcal{R}^{l} u \in b_{q+p l}^{p}$.

Proof. There is nothing to prove for $(\mathrm{b}) \Rightarrow(\mathrm{c})$ and $(\mathrm{d}) \Rightarrow(\mathrm{e})$.

(a) $\Rightarrow(\mathrm{b})$ Let $u \in b_{q}^{p}$. By Lemma 12.1, for $i, j=1, \ldots, n$, first $\partial_{i} u \in b_{q+p}^{p}$, then $\partial_{i j}^{2} u \in b_{q+2 p}^{p}$, and so on until $\partial^{\alpha} u$ is obtained.

(c) $\Rightarrow$ (a) Let $\beta$ be any multi-index with $|\beta|=l-1$. By hypothesis, we have $\partial_{i} \partial^{\beta} u \in b_{q+p l}^{p}$. Then $\partial^{\beta} u \in b_{q+p(l-1)}^{p}$ by Lemma 12.1. We repeat until $u \in b_{q}^{p}$ is obtained.

(a) $\Rightarrow(d)$ is almost the same as $(\mathrm{a}) \Rightarrow(\mathrm{b})$, and $(\mathrm{e}) \Rightarrow(\mathrm{a})$ is almost the same as (c) $\Rightarrow(\mathrm{a})$.

For $q=0$, what we have just proved appears in [7, Theorem 1.3].

Lemma 12.3. Given an $l=1,2, \ldots$, for multi-indices $\alpha$ with $|\alpha| \leq l$, there are polynomials $f_{\alpha}$ with $\operatorname{deg}\left(f_{\alpha}\right)=|\alpha|$ such that $\mathcal{R}^{l}=\sum_{|\alpha| \leq l} f_{\alpha} \partial^{\alpha}$. 
Proof. Let $u \in h(\mathbb{B})$. For $l=1,(2.6)$ shows $\mathcal{R} u=\sum_{i=1}^{n} x_{i} \partial_{i} u$. For $l=2$,

$$
\mathcal{R}^{2} u=\sum_{j=1}^{n} x_{j} \partial_{j}\left(\sum_{i=1}^{n} x_{i} \partial_{i} u\right)=\sum_{i, j=1}^{n} x_{i} x_{j} \partial_{i j}^{2} u+\sum_{j=1}^{n} x_{j} \partial_{j} u,
$$

as claimed. The general case is obtained by induction on $l$ and the induction step is no different from obtaining $l=2$ from $l=1$, only more cumbersome.

Lemma 12.4. Suppose $q>-1$ and $u \in h(\mathbb{B})$. If $\partial^{\alpha} u \in b_{q}^{p}$ for every multi-index $\alpha$ with $|\alpha|=l$, then $\partial^{\beta} u \in b_{q}^{p}$ for all multi-indices $\beta$ with $|\beta| \leq l$.

Proof. If $\partial^{\alpha} u \in b_{q}^{p}$ for every $\alpha$ with $|\alpha|=l$ and $|\beta|<l$, then by Lemma 12.2, $\partial^{\beta}\left(\partial^{\alpha} u\right) \in b_{q+p|\beta|}$. Hence $\partial^{\alpha}\left(\partial^{\beta} u\right) \in b_{q+p l}$ for every $\alpha$ chosen, by Corollary 9.1. By Lemma 12.2 again, $\partial^{\beta} u \in b_{q}^{p}$.

We are finally ready to prove Theorem 1.2 about the interchangeability of various kinds of derivatives in defining harmonic Besov spaces under the condition (1.2).

Proof of Theorem 1.2. Only three implications need to be proved.

$(\mathrm{a}) \Rightarrow$ (b) In the light of Theorem 1.1, the hypothesis allows us to pick a pair $s, t$ satisfying (1.2), $D_{s}^{t} u \in b_{q+p t}^{p}$, which is a Bergman space. Choose $b$ so large that $q+p t+1<p(b+1)$ and $b>t$. By $(1.5)$,

$$
D_{s}^{t} u(x)=\int_{\mathbb{B}} D_{s}^{t} u(y) R_{b}(x, y) d \nu_{b}(y) .
$$

Let $\alpha$ be any multi-index with $l=|\alpha|$ and $q+p l>-1$. Apply $D_{s+t}^{-t}$ and then $\partial^{\alpha}$ in the $x$ variable and differentiate under the integral sign; the result is

$$
\partial^{\alpha} u(x)=\int_{\mathbb{B}} D_{s}^{t} u(y) \partial^{\alpha} D_{s+t}^{-t} R_{b}(x, y) d \nu_{b}(y) .
$$

Now $D_{s+t}^{-t} R_{b}$ has the form of $W$ in Lemma 7.4, and thus we can estimate growth rate of its derivative by this lemma. Using Definitions 3.1 and 3.2 , that $b>t$ and (2.4), we obtain

$$
\begin{gathered}
\left|\partial^{\alpha} u(x)\right| \lesssim \int_{\mathbb{B}}\left|D_{s}^{t} u(y)\right| \frac{1}{\{x, y\}^{(n+b-t+l) / 2}} d \nu_{b}(y), \\
\left(1-|x|^{2}\right)^{l}\left|\partial^{\alpha} u(x)\right| \lesssim\left(1-|x|^{2}\right)^{l} \int_{\mathbb{B}}\left(1-|y|^{2}\right)^{t}\left|D_{s}^{t} u(y)\right| \frac{\left(1-|y|^{2}\right)^{b-t}}{\{x, y\}^{(n+b-t+l) / 2}} d \nu(y) .
\end{gathered}
$$

Applying Theorem 1.6 now yields $\left(1-|x|^{2}\right)^{l}\left|\partial^{\alpha} u(x)\right| \in L_{q}^{p}$.

(c) $\Rightarrow$ (d) Let $l_{0} \in \mathbb{N}$ be the integer provided by (c) so that $\partial^{\alpha} u \in b_{q+p l_{0}}^{p}$ for every $\alpha$ with $|\alpha|=l_{0}$. By Lemma $12.4, \partial^{\beta} u \in b_{q+p l_{0}}^{p}$ for all $\beta$ with $|\beta| \leq l_{0}$. Then by Lemma $12.3, \mathcal{R}^{l_{0}} u \in b_{q+p l_{0}}^{p}$. Notice that $b_{q+p l_{0}}^{p}$ is a Bergman space.

Now let $l \in \mathbb{N}$ satisfy $q+p l>-1$ but be otherwise arbitrary. If $l>l_{0}$, then $\mathcal{R}^{l} u=\mathcal{R}^{l-l_{0}} \mathcal{R}^{l_{0}} u \in b_{q+p l}^{p}$ by the previous paragraph and Lemma 12.2. If $l<l_{0}$, 
then $\mathcal{R}^{l_{0}} u=\mathcal{R}^{l_{0}-l} \mathcal{R}^{l} u \in b_{q+p l_{0}}^{p}$ again by the previous paragraph. Then $\mathcal{R}^{l} u \in b_{q+p l}^{p}$ by Theorem 12.2, because $b_{q+p l}^{p}$ is also a Bergman space.

(e) $\Rightarrow$ (a) Choose $s, t$ satisfying (1.2); so $q+p t>-1$ and $b_{q+p t}^{p}$ is a Bergman space. By Corollary 9.2, $D_{s}^{t}\left(\mathcal{R}^{l} u\right) \in b_{q+p l+p t}^{p}$. But $D_{s}^{t}$ and $\mathcal{R}^{l}$ commute as they act on the homogeneous expansion of $u$ here. So by Lemma 12.2, $D_{s}^{t} u \in b_{q+p t}^{p}$. By Theorem 1.1, $u \in b_{q}^{p}$.

The part of this result utilizing partial derivatives appears in [24, Theorem 3(a)] for $p>1$ with a different proof. It also appears in [15, Theorem 3.2] for only $q=-n$.

\section{Applications of Projections}

We extract from Theorem 1.4 and the integral representations (1.5) many properties of the spaces $b_{q}^{p}$.

\subsection{Growth of functions and Fourier coefficients}

Theorem 13.1. We have uniform growth rates for all $u \in b_{q}^{p}$ as $x$ approaches the boundary of $\mathbb{B}$ :

$$
|u(x)| \lesssim\|u\|_{b_{q}^{p}} \begin{cases}1 & \text { if } q<-n \\ \left(\frac{1}{|x|^{2}} \log \frac{1}{1-|x|^{2}}\right)^{1-1 / p} & \text { if } q=-n \\ \frac{1}{\left(1-|x|^{2}\right)^{(n+q) / p}} & \text { if } q>-n .\end{cases}
$$

Note that when $p=1$, the cases $q=-n$ and $q<-n$ are the same. Note also that $1-1 / p=1 / p^{\prime}$.

Proof. Choose $s, t$ in accordance with (1.3) and (1.2). First let $p>1$. Recall the integral representation (1.5). Write the measure as $\nu_{q}$ and apply Hölder inequality to the resulting form. Then

$$
|u(x)| \lesssim\|u\|_{b_{q}^{p}}\left(\int_{\mathbb{B}}\left|R_{s}(x, y)\right|^{p^{\prime}}\left(1-|y|^{2}\right)^{q+(-q+s) p^{\prime}} d \nu(y)\right)^{1 / p^{\prime}} .
$$

Now the exponent $q+(-q+s) p^{\prime}>-1$ by (1.3), the quantity $c$ in Theorem 1.5 is $c=(n+q)\left(p^{\prime}-1\right)$, and $c$ changes sign at $q=-n$. Using the estimates in Theorem 1.5 and taking $1 / p^{\prime}$ powers, we obtain all the growth rates at once.

For $p=1$, (1.3) says $q<s$, but let additionally $s>-n$ for convenience. By (1.5), we first have

$$
|u(x)| \lesssim\|u\|_{b_{q}^{1}} \max \left\{\left|R_{s}(x, y)\right|\left(1-|y|^{2}\right)^{-q+s}: y \in \mathbb{B}\right\} .
$$

By Proposition 3.2,

$$
\left|R_{s}(x, y)\right|\left(1-|y|^{2}\right)^{-q+s} \lesssim \frac{\left(1-|y|^{2}\right)^{-q+s}}{(1-|x||y|)^{n+s}} \lesssim \frac{1}{(1-|x||y|)^{n+q}} \quad(x, y \in \mathbb{B}) .
$$


The last term is clearly bounded if $q \leq-n$. If $q>-n$, then the last term is $\lesssim \frac{1}{\left(1-|x|^{2}\right)^{n+q}}$.

The case $q=-1$ and $p=2$ is in [1, Proposition 6.23$]$, and the case $q=0$ is in [1, Proposition 8.1].

Corollary 13.1. Derivatives of functions in $b_{q}^{p}$ also have uniform growth rates as $|x| \rightarrow 1^{-}$. For any $s, t \in \mathbb{R}$,

$$
\left|D_{s}^{t} u(x)\right| \lesssim\|u\|_{b_{q}^{p}} \begin{cases}1 & \text { if } q+p t<-n ; \\ \left.\frac{1}{|x|^{2}} \log \frac{1}{1-|x|^{2}}\right)^{1-1 / p} & \text { if } q+p t=-n ; \\ \frac{1}{\left(1-|x|^{2}\right)^{(n+q+p t) / p}} & \text { if } q+p t>-n .\end{cases}
$$

Proof. It is enough to apply Theorem 13.1 and Corollary 9.2 together.

Corollary 13.2. Point evaluations are bounded linear functionals on $b_{q}^{p}$. The same is true for the functionals $u \mapsto D_{s}^{t} u(x)$ for any $x \in \mathbb{B}$ and $s, t$. Furthermore, for each choice of $K \subset \mathbb{B}$ compact, $s, t$, and $q, p$, we have

$$
\sup \left\{\left|D_{s}^{t} u(x)\right|: x \in K\right\} \lesssim\|u\|_{b_{q}^{p}} .
$$

Proof. It suffices to note that $1-|x|^{2} \sim 1$ for $x \in K$.

We can prove that a little more than Theorem 13.1 is true in different ways for $q>-n$ and $q<-n$.

Proposition 13.1. If $q>-n$ and $u \in b_{q}^{p}$, then

$$
\lim _{|x| \rightarrow 1^{-}}\left(1-|x|^{2}\right)^{(n+q) / p}|u(x)|=0 .
$$

Proof. Apply the relevant part of Theorem 13.1 to $u-u_{\tau}$, where $u_{\tau}$ is the dilate of $u$, and multiply both sides by $\left(1-|x|^{2}\right)^{(n+q) / p}$. Let $\varepsilon>0$. By Theorem 4.2 , there is a $0<\tau_{0}<1$ such that for $\tau_{0} \leq \tau<1$, we have $\left(1-|x|^{2}\right)^{(n+q) / p}\left|u(x)-u_{\tau}(x)\right|<\varepsilon$ for $x \in \mathbb{B}$. Then $\left(1-|x|^{2}\right)^{(n+q) / p}|u(x)|<\varepsilon+\left(1-|x|^{2}\right)^{(n+q) / p}\left|u_{\tau_{0}}(x)\right|$ for $x \in \mathbb{B}$. Now we let $|x| \rightarrow 1^{-}$note that $u_{\tau_{0}}$ is bounded in $\overline{\mathbb{B}}$.

Theorem 13.2. When $q<-n$, all the spaces $b_{q}^{p}$ lie in $h^{\infty}$, and so does $b_{-n}^{1}$, with continuous inclusion. Furthermore, functions in $b_{q}^{p}$ with $q<-n$ extend continuously to $\overline{\mathbb{B}}$.

Proof. The first claim is a restatement of the relevant parts of Theorem 13.1.

For the second claim, let $u \in b_{q}^{p}$ with $q<-n$, and pick $t$ satisfying (1.2); so $t>0$. Let $s=0$ for convenience; then (1.3) obtains and (1.5) yields

$$
u(x)=\frac{1}{V_{t}} \int_{\mathbb{B}} D_{0}^{t} u(y) R_{0}(x, \eta)\left(1-|y|^{2}\right)^{t} d \nu(y) .
$$


Call the integral of the absolute value of the integrand $M(x)$ on which we use Corollaries 13.1 and 7.1. Then

$$
M(x) \lesssim \int_{\mathbb{B}}\left(1-|y|^{2}\right)^{-(n+q) / p} \frac{1}{\{x, y\}^{n / 2}} d \nu(y),
$$

to which we apply Remark 6.1 with Lemma 6.2 . Thus the integral for $u(x)$ is absolutely and uniformly convergent for $x \in \overline{\mathbb{B}}$. Letting $x \rightarrow \xi \in \partial \mathbb{B}$, we see that

$$
u(\xi)=\frac{1}{V_{t}} \int_{\mathbb{B}} D_{0}^{t} u(y) R_{0}(\xi, y)\left(1-|y|^{2}\right)^{t} d \nu(y)
$$

is a continuous extension.

Theorem 13.3. Let $n=2, u \in b_{q}^{p}$, and let $c_{m}$ be the Fourier coefficients of $u$ as defined in (14.11), where $m \in \mathbb{Z}$ with notation specific to $n=2$ as explained at the end of Sec. 5. Then

$$
\left|c_{m}\right| \lesssim\|u\|_{b_{q}^{p}}|m|^{(1+q) / p}
$$

Proof. Let $s, t$ be chosen conforming to (1.3) and (1.2). We first note a formula for the coefficients $c_{m k}$ for general $n \geq 2$ :

$$
c_{m k}=\gamma_{m}(s) \int_{\mathbb{D}} D_{s}^{t} u(y) \overline{Y_{m k}(y)} d \nu_{s+t} .
$$

This is proved by expanding $u$ into a series as in (14.11), using Definition 3.2, the orthonormality of $\left\{Y_{m k}\right\}$, and (5.1). At this point we have to restrict to $n=2$, because otherwise we do not know explicit forms of the $Y_{m k}$. So for $m \in \mathbb{Z}$,

$$
\left|c_{m}\right| \leq \gamma_{|m|}(s) \int_{\mathbb{D}}\left|D_{s}^{t} u(y)\right||y|^{|m|} d \nu_{s+t} .
$$

Note that $s+t>-1$.

We write the measure as $\nu_{q}$ and change $D_{s}^{t}$ to $I_{s}^{t}$, apply Hölder inequality, evaluate the resulting integral with polar coordinates and the beta function, use (3.5), and (2.1). What we obtain is

$$
\begin{aligned}
\left|c_{m}\right| & \lesssim \gamma_{|m|}(s)\|u\|_{b_{q}^{p}}\left(\int_{\mathbb{D}}|y|^{|m| p^{\prime}}\left(1-|y|^{2}\right)^{q+p^{\prime}(s-q)} d \nu(y)\right)^{1 / p^{\prime}} \\
& \sim\|u\|_{b_{q}^{p}}|m|^{1+s}\left(\int_{0}^{1} \rho^{1+p^{\prime}|m|}\left(1-\rho^{2}\right)^{q+p^{\prime}(s-q)} d \rho\right)^{1 / p^{\prime}} \\
& \sim\|u\|_{b_{q}^{p}}|m|^{1+s}\left(\frac{\Gamma\left(1+p^{\prime}|m| / 2\right)}{\Gamma\left(2+q+p^{\prime}(s-q)+p^{\prime}|m| / 2\right)}\right)^{1 / p^{\prime}} \\
& \sim\|u\|_{b_{q}^{p}}|m|^{1+s}|m|^{-(1+q) / p^{\prime}-(s-q)} \\
& \sim\|u\|_{b_{q}^{p}|m|^{(1+q) / p}}
\end{aligned}
$$


Remark 13.1. Classical Fourier coefficients are known to have limit 0 as their index tends to $\pm \infty$, because they belong to integrable functions on $\partial \mathbb{D}$. The generalized Fourier coefficients here are not those of such functions in general; for $q>-1$, functions in $b_{q}^{p}$ need not even have any boundary values. But when $q<-1$, at least for $p=2$, the spaces $b_{q}^{2}$ all lie in the Hardy space $h^{2}=b_{-1}^{2}$ and hence functions in them do have integrable boundary values. So their Fourier coefficients must tend to 0 as $|m| \rightarrow \infty$, which is exactly what Theorem 13.3 says for $q<-1$.

\subsection{Inclusions}

We find which Besov spaces are included in what other Besov spaces. Our first two results do not require Bergman projections and could have been proved in Sec. 9, but we put them here for the unity of presentation.

Proposition 13.2. (a) If $q_{1}<q_{2}$, then $b_{q_{1}}^{p} \subset b_{q_{2}}^{p}$, and the associated inclusion map $i$ is bounded with norm at most $V_{q_{1}} / V_{q_{2}}$ with suitable $s, t$ used in norms.

(b) If $q>-1$ and $p_{1} \leq p_{2}$, then $b_{q}^{p_{2}} \subset b_{q}^{p_{1}}$, and the associated inclusion map is bounded with norm at most 1 with $t=0$ in norms.

(c) Let $p_{1} \leq p_{2}$ and suppose $q_{1}, q_{2}$ satisfy $\frac{q_{2}-q}{p_{2}} \leq \frac{q_{1}-q}{p_{1}}$ for some $q>-1$. Then $b_{q_{2}}^{p_{2}} \subset b_{q_{1}}^{p_{1}}$ with bounded inclusion map. In particular, this inclusion holds if $q_{2} p_{1} \leq q_{1} p_{2}$.

Proof. (a) This is just Corollary 9.1 repeated here for completeness.

(b) This holds because now $\nu_{q}$ is finite. These spaces are precisely the harmonic Bergman spaces.

(c) Call the fractions $-t_{2}$ and $-t_{1}$. Then $q_{1}+p_{1} t_{1}=q_{2}+p_{2} t_{2}=q>-1$. Hence $D_{s}^{t_{2}}\left(b_{q_{2}}^{p_{2}}\right)=b_{q_{2}+p_{2} t_{2}}^{p_{2}}=b_{q}^{p_{2}} \subset b_{q}^{p_{1}}=b_{q_{1}+p_{1} t_{1}}^{p_{1}}$ by Corollary 9.2 and (b). Applying $D_{s+t_{2}}^{-t_{2}}$ to both sides, using (3.9), Corollary 9.2 once again, and (a), we finally obtain $b_{q_{2}}^{p_{2}} \subset b_{q_{1}+p_{1}\left(t_{1}-t_{2}\right)}^{p_{1}} \subset b_{q_{1}}^{p_{1}}$ since $t_{1} \leq t_{2}$.

Corollary 13.3. Besov spaces $b_{q}^{p}$ with different $q$ and same $p$ are different.

Proof. Let $q_{1}<q_{2}$; then the inclusion map $i_{12}: b_{q_{1}}^{p} \rightarrow b_{q_{2}}^{p}$ is bounded by Proposition 13.2(a). If $b_{q_{1}}^{p}=b_{q_{2}}^{p}$ did hold, then $i_{21}: b_{q_{2}}^{p} \rightarrow b_{q_{1}}^{p}$ would also be bounded by the closed graph theorem. Let $u_{m} \in \mathcal{H}_{m}$. Then by (4.2),

$$
\frac{\left\|u_{m}\right\|_{b_{q_{1}}^{p}}}{\left\|u_{m}\right\|_{b_{q_{2}}^{p}}} \sim m^{\left(q_{2}-q_{1}\right) / p} \rightarrow \infty \quad(m \rightarrow \infty)
$$

contradicting the boundedness of $i_{21}$.

Proposition 13.3. (a) If $u \in b_{-n}^{p}$ and $t>0$, then $\sup _{x \in \mathbb{B}}\left|I_{s}^{t} u(x)\right| \lesssim\|u\|_{b_{-n}^{p}}$.

(b) If $p_{1} \leq p_{2}$, then $b_{-n}^{p_{1}} \subset b_{-n}^{p_{2}}$ with bounded inclusion map.

(c) Let $p_{1} \leq p_{2}$ and suppose $q_{1}, q_{2}$ satisfy $\frac{q_{1}+n}{p_{1}} \leq \frac{q_{2}+n}{p_{2}}$. Then $b_{q_{1}}^{p_{1}} \subset b_{q_{2}}^{p_{2}}$ with bounded inclusion map. 
Proof. (a) Apply the relevant part of Corollary 13.1 with $q=-n$.

(b) Let $s, t$ satisfy (1.2) with $q=-n$; clearly $t>0$. We directly compute

$$
\begin{aligned}
\|u\|_{b_{-n}^{p_{2}}}^{p_{2}} & =\int_{\mathbb{B}}\left|I_{s}^{t} u\right|^{p_{2}} d \nu_{-n}=\int_{\mathbb{B}}\left|I_{s}^{t} u\right|^{p_{1}}\left|I_{s}^{t} u\right|^{p_{2}-p_{1}} d \nu_{-n} \\
& \lesssim\|u\|_{b_{-n}^{p_{1}}}^{p_{2}-p_{1}} \int_{\mathbb{B}}\left|I_{s}^{t} u\right|^{p_{1}} d \nu_{-n}=\|u\|_{b_{-n}^{p_{1}}}^{p_{2}-p_{1}}\|u\|_{b_{-n}^{p_{1}}}^{p_{1}}=\|u\|_{b_{-n}^{p_{1}}}^{p_{2}}
\end{aligned}
$$

using (a).

(c) Call the two fractions $-t_{1}$ and $-t_{2}$. Then $q_{1}+p_{1} t_{1}=q_{2}+p_{2} t_{2}=-n$. Hence $D_{s}^{t_{2}}\left(b_{q_{2}}^{p_{2}}\right)=b_{q_{2}+p_{2} t_{2}}^{p_{2}}=b_{-n}^{p_{2}} \supset b_{-n}^{p_{1}}=b_{q_{1}+p_{1} t_{1}}^{p_{1}}$ by Corollary 9.2 and (b). Applying $D_{s+t_{2}}^{-t_{2}}$ to both sides, by (3.9), Corollary 9.2 again, and (b), we obtain $b_{q_{2}}^{p_{2}} \supset b_{q_{1}+p_{1}\left(t_{1}-t_{2}\right)}^{p_{1}} \supset b_{q_{1}}^{p_{1}}$ since $t_{1} \geq t_{2}$.

Note that the inclusions in parts (c) of Propositions 13.2 and 13.3 are in opposite directions. The holomorphic counterpart of the latter is [2, Theorem 5.13]. If equality holds in the two fractions in these two parts, call their common value $\lambda$. Then $q_{1}=q+\lambda p_{1}$ and $q_{2}=q+\lambda p_{2}$ with $q>-1$ in Proposition 13.2(c) and $q=-n$ in Proposition 13.3(c). These are rays in the right half $p q$-plane with slope $\lambda \in \mathbb{R}$ and $q$-intercept $q>-1$ and $q=-n$ in the two propositions, respectively. So if two Besov spaces lie on the section $p \geq 1$ of one of these rays, then one is included in the other the way just described.

\subsection{Duality}

We now identify the duals of the spaces $b_{q}^{p}$ and they turn out to be $b_{q}^{p^{\prime}}$ as expected. However, the pairings under they are realized are quite varied. As we have done from the very beginning, we restrict ourselves to $p<\infty$. The proofs make essential use of Theorem 1.4 and its corollaries.

Theorem 13.4. For given $q$ and $1<p<\infty$, if $s, t$ satisfy (1.3) and (1.2), the dual of $b_{q}^{p}$ can be identified with $b_{q}^{p^{\prime}}$ under each of the pairings

$$
[u, v]_{b_{q}^{2}}:=\left[I_{s}^{t} u, I_{q+t}^{-q+s} v\right]_{L_{q}^{2}}=\int_{\mathbb{B}} I_{s}^{t} u \overline{I_{q+t}^{-q+s} v} d \nu_{q}
$$

Proof. Under (1.2) and (1.3), the similar inequalities

$$
q+p^{\prime}(-q+s)>-1 \text { and } q+1<p^{\prime}(q+t+1)
$$

hold. So if $v \in b_{q}^{p^{\prime}}$, then $I_{q+t}^{-q+s} v \in L_{q}^{p^{\prime}}$ by Definition 1.1, and $M_{v}=[u, v]_{b_{q}^{2}}$ defines a bounded linear functional on $b_{q}^{p}$ of norm at most $\left\|I_{q+t}^{-q+s} v\right\|_{L_{q}^{p^{\prime}}}$ by Hölder inequality.

Conversely, let $M$ be a bounded linear functional on $b_{q}^{p}$. Recalling Remark 11.2, let $\widetilde{Q}_{s}$ be the restriction of $Q_{s}$ to $I_{s}^{t}\left(b_{q}^{p}\right)$. Then $M \widetilde{Q}_{s}$ is a bounded linear functional on $I_{s}^{t}\left(b_{q}^{p}\right)$. By the Hahn-Banach theorem, it extends to a linear functional $L$ on $L_{q}^{p}$ with $\|L\|=\left\|M \widetilde{Q}_{s}\right\|$. By the Riesz representation theorem, there is a unique $g \in L_{q}^{p^{\prime}}$ 
with $\|g\|_{L_{q}^{p^{\prime}}}=\left\|M \widetilde{Q}_{s}\right\|$ such that $L(f)=[f, g]_{L_{q}^{2}}$. In particular, when $f=I_{s}^{t} u$ for $u \in b_{q}^{p}$, this last form becomes $M Q_{s} I_{s}^{t}(u)=M \widetilde{Q}_{s} I_{s}^{t}(u)=\left[I_{s}^{t} u, g\right]_{L_{q}^{2}}$. By repeated use of Corollary 11.1 and Lemma 8.1, we equivalently have

$$
\begin{aligned}
M(u) & =\frac{V_{s}}{V_{s+t}}\left[I_{s}^{t} u, g\right]_{L_{q}^{2}}=\frac{V_{s}}{V_{s+t}^{2}}\left[T_{s, t} I_{s}^{t} u, g\right]_{L_{q}^{2}}=\frac{V_{s}}{V_{s+t}^{2}}\left[I_{s}^{t} u, T_{q+t,-q+s} g\right]_{L_{q}^{2}} \\
& =\frac{V_{s} V_{q+t}}{V_{s+t}^{2}}\left[I_{s}^{t} u, I_{q+t}^{-q+s} Q_{q+t} g\right]_{L_{q}^{2}}=\left[I_{s}^{t} u, I_{q+t}^{-q+s} v\right]_{L_{q}^{2}},
\end{aligned}
$$

where $v:=\frac{V_{s} V_{q+t}}{V_{s+t}^{2}} Q_{q+t} g \in b_{q}^{p^{\prime}}$ by (13.1) and Theorem 1.4. Uniqueness of $v$ follows from the uniqueness of $g$.

Thus the harmonic Besov spaces $b_{q}^{p}$ for $1<p<\infty$ are reflexive.

See [16, Corollary 4.3] for $q=0$ but on smooth bounded domains, [32, Theorem 3.1] for $0<q<p-1$ but for vector-valued spaces, [4, Corollary 2] for Bergman spaces with general weights, [28, Corollary 5.1] for $q>-1$, and [14, Theorem 3.2] for $q>-1$ again. The result in [15, Theorem 5.1] is an exception in that it has $q=-n$. Note that we have $q \in \mathbb{R}$ without restriction.

The duality result in [23, Theorem 2(a)] is of a different character. The dual of a harmonic Bergman space $b_{q}^{p}$ with $q \geq 0$ is identified with a Besov space $b_{q_{1}}^{p^{\prime}}$ with $q_{1} \leq 0$. Thus in its special case [22, Theorem 2], the dual of a Hilbert space is identified with a different Hilbert space.

Now that we know the duals of $b_{q}^{p}$, we identify the adjoints $Q_{s}^{*}: b_{q}^{p^{\prime}} \rightarrow L_{q}^{p^{\prime}}$ of the bounded Bergman-Besov projections $Q_{s}: L_{q}^{p} \rightarrow b_{q}^{p}$ for $1<p<\infty$ with respect to the pairings $\left[f, Q_{s}^{*} u\right]_{L_{q}^{2}}=\left[Q_{s} f, u\right]_{b_{q}^{2}}:=\left[I_{s}^{t} Q_{s} f, I_{q+t}^{-q+s} u\right]_{L_{q}^{2}}$ in the statement of Theorem 13.4, where $f \in L_{q}^{p}, u \in b_{q}^{p^{\prime}}$, and $s, t$ satisfy (1.3) and (1.2). As noted in the proof of Theorem 13.4, $I_{q+t}^{-q+s} u \in L_{q}^{p^{\prime}}$.

Corollary 13.4. $Q_{s}^{*}=\frac{V_{s+t}}{V_{s}} I_{q+t}^{-q+s}$ and $\left(I_{s}^{t}\right)^{*}=\frac{V_{q+t}}{V_{s+t}} Q_{q+t}$.

Proof. By Corollary 11.1, we have

$$
\begin{aligned}
{\left[Q_{s} f, u\right]_{b_{q}^{p}} } & =\left[I_{s}^{t} Q_{s} f, I_{q+t}^{-q+s} u\right]_{L_{q}^{2}}=\frac{1}{V_{s}}\left[T_{s, t} f, I_{q+t}^{-q+s} u\right]_{L_{q}^{2}} \\
& =\frac{1}{V_{s}}\left[f, T_{s, t}^{*} I_{q+t}^{-q+s} u\right]_{L_{q}^{2}}=\frac{1}{V_{s}}\left[f, T_{q+t,-q+s} I_{q+t}^{-q+s} u\right]_{L_{q}^{2}} \\
& =\frac{V_{s+t}}{V_{s}}\left[f, I_{q+t}^{-q+s} u\right]_{L_{q}^{2}} .
\end{aligned}
$$

\subsection{Complex interpolation of spaces}

Again, we are content with results that make no use of the case $p=\infty$. Again, the essential ingredients of the proofs are the projections $Q_{s}$ and the imbeddings $I_{s}^{t}$. 
For general information on complex interpolation between Banach spaces, see [44, Sec. 1.8]. Any two harmonic Besov spaces are compatible, because they are contained in Bergman spaces with sufficiently large same $q$ and possibly different $p$, and thus in the Bergman space $b_{q}^{1}$; this is by Proposition 13.2(a),(b). We use the notation $\langle X, Y\rangle_{\theta}$ for the complex interpolation space between the spaces $X$ and $Y$, where $0 \leq \theta \leq 1$.

Suppose $0 \leq \theta \leq 1$, the exponents are related by

$$
\frac{1}{p}=\frac{1-\theta}{p_{0}}+\frac{\theta}{p_{1}} \quad\left(1 \leq p_{0}, p_{1}, p<\infty\right),
$$

and the weights of the Lebesgue classes are related by

$$
\left(1-|x|^{2}\right)^{q / p}=\left(1-|x|^{2}\right)^{(1-\theta) q_{0} / p_{0}}\left(1-|x|^{2}\right)^{\theta q_{1} / p_{1}},
$$

that is, by

$$
\frac{q}{p}=\frac{(1-\theta) q_{0}}{p_{0}}+\frac{\theta q_{1}}{p_{1}} \quad\left(q_{0}, q_{1}, q \in \mathbb{R}\right) .
$$

We consider $p_{0}, p_{1}, q_{0}, q_{1}$ as given and $p, q$ depending on $\theta$. Then by $[3$, Theorem 5.5.1], we have

$$
\left\langle L_{q_{0}}^{p_{0}}, L_{q_{1}}^{p_{1}}\right\rangle_{\theta}=L_{q}^{p}
$$

Theorem 13.5. Suppose (13.2) and (13.3) hold. Then $\left\langle b_{q_{0}}^{p_{0}}, b_{q_{1}}^{p_{1}}\right\rangle_{\theta}=b_{q}^{p}$.

Proof. Choose $s$ large so that (1.3) is satisfied with the pairs $\left(q_{0}, p_{0}\right),\left(q_{1}, p_{1}\right)$, and $(q, p)$ for all $0 \leq \theta \leq 1$. Then by Theorem 1.4, the projection $Q_{s}$ is bounded from the Lebesgue classes with these parameters onto the Besov spaces with the same parameters. By [3, Corollary 5.5.4] and (13.4), $Q_{s}$ maps $\left\langle L_{q_{0}}^{p_{0}}, L_{q_{1}}^{p_{1}}\right\rangle_{\theta}=L_{q}^{p}$ into $\left\langle b_{q_{0}}^{p_{0}}, b_{q_{1}}^{p_{1}}\right\rangle_{\theta}$. The use of onto and into above shows that $b_{q}^{p} \subset\left\langle b_{q_{0}}^{p_{0}}, b_{q_{1}}^{p_{1}}\right\rangle_{\theta}$.

Conversely, choose $t$ so large that (1.2) is satisfied with the parameters $\left(q_{0}, p_{0}\right)$, $\left(q_{1}, p_{1}\right)$, and $(q, p)$ for all $0 \leq \theta \leq 1$. Then the imbedding $I_{s}^{t}$ maps the Besov spaces with these parameters into the Lebesgue spaces with the same parameters by Definition 1.1. Then by [3, Corollary 5.5.4] and (13.4), $I_{s}^{t}$ maps $\left\langle b_{q_{0}}^{p_{0}}, b_{q_{1}}^{p_{1}}\right\rangle_{\theta}$ into $\left\langle L_{q_{0}}^{p_{0}}, L_{q_{1}}^{p_{1}}\right\rangle_{\theta}=L_{q}^{p}$. By Definition 1.1 again, this means that $\left\langle b_{q_{0}}^{p_{0}}, b_{q_{1}}^{p_{1}}\right\rangle_{\theta} \subset b_{q}^{p}$.

This theorem is not new; it appears in [25, Theorem 2], in even a more general form for Sobolev (Besov) spaces of harmonic functions on smooth bounded domains in $\mathbb{R}^{n}$. On the other hand, the Sobolev spaces of this source are already defined using complex interpolation between those of integer order circumventing the difficulties of interpolation.

Corollary 13.5. Suppose (13.2) holds. Then $\left\langle b_{q}^{p_{0}}, b_{q}^{p_{1}}\right\rangle_{\theta}=b_{q}^{p}$.

Corollary 13.6. Suppose $q=(1-\theta) q_{0}+\theta q_{1}$. Then $\left\langle b_{q_{0}}^{p}, b_{q_{1}}^{p}\right\rangle_{\theta}=b_{q}^{p}$. 


\subsection{Gleason problem}

In the context of the harmonic Besov spaces, the Gleason problem is this: Given $u \in$ $b_{q}^{p}$ and $x^{0} \in \mathbb{B}$, find $u_{1}, \ldots, u_{n} \in b_{q}^{p}$ such that $u(x)-u\left(x^{0}\right)=\sum_{i=1}^{n}\left(x_{i}-x_{i}^{0}\right) u_{i}(x)$ for all $x \in \mathbb{B}$. We exhibit a solution to this problem based on the integral representations ensuing from Bergman-Besov projections.

Theorem 13.6. Given $q, p$ and $x^{0} \in \mathbb{B}$, there exist bounded linear operators $A_{1}, \ldots, A_{n}: b_{q}^{p} \rightarrow b_{q}^{p}$ such that

$$
u(x)-u\left(x^{0}\right)=\sum_{i=1}^{n}\left(x_{i}-x_{i}^{0}\right) A_{i} u(x) \quad\left(u \in b_{q}^{p}, x \in \mathbb{B}\right) .
$$

Proof. For $x \in \mathbb{B}$ and $0 \leq \tau \leq 1$, put $z=\tau x+(1-\tau) x^{0} \in \mathbb{B}$. It is an elementary application of the fundamental theorem of calculus that

$$
\begin{aligned}
u(x)-u\left(x^{0}\right) & =\int_{0}^{1} \frac{d}{d \tau}(u(z)) d \tau=\int_{0}^{1}\left(x-x^{0}\right) \cdot \nabla u(z) d \tau \\
& =\sum_{i=1}^{n}\left(x_{i}-x_{i}^{0}\right) \int_{0}^{1} \partial_{i} u(z) d \tau \quad(x \in \mathbb{B})
\end{aligned}
$$

for any continuously differentiable $u$ on $\mathbb{B}$. We define $A_{i} u(x)$ to be the integral in (13.5); thus the $A_{i} u$ satisfy the equation required for a solution of the Gleason problem. If $u \in h(\mathbb{B})$, then also $A_{i} u \in h(\mathbb{B})$ by differentiation under the integral sign and the chain rule with a linear change of variables. It remains to show that $A_{i} u \in b_{q}^{p}$ whenever $u \in b_{q}^{p}$, and this is the main part of the proof.

Pick $l \in \mathbb{N}$ with $q+p l>-1$ and let $\alpha$ be a multi-index such that $|\alpha|=l$. By Theorem 1.2, it suffices to show that $\left(1-|x|^{2}\right)^{l}\left(\partial^{\alpha} A_{i} u\right)(x) \in L_{q}^{p}$.

Choose $s, t$ satisfying (2), (3), and also $s>-1$. By (1.5),

$$
u(x)=\frac{1}{V_{s+t}} \int_{\mathbb{B}} I_{s}^{t} u(y) R_{s}(x, y)\left(1-|y|^{2}\right)^{s} d \nu(y)
$$

and hence

$$
A_{i} u(x)=\frac{1}{V_{s+t}} \int_{0}^{1} \int_{\mathbb{B}} I_{s}^{t} u(y) \partial_{i} R_{s}(z, y)\left(1-|y|^{2}\right)^{s} d \nu(y) d \tau .
$$

Differentiating under the integral sign and using the chain rule, we obtain

$$
\partial^{\alpha} A_{i} u(x)=\frac{1}{V_{s+t}} \int_{0}^{1} \tau^{l} \int_{\mathbb{B}} I_{s}^{t} u(y) \partial^{\alpha} \partial_{i} R_{s}(z, y)\left(1-|y|^{2}\right)^{s} d \nu(y) d \tau .
$$

An application of the Fubini theorem and Corollary 7.1 yields

$$
\left(1-|x|^{2}\right)^{l}\left|\partial^{\alpha} A_{i} u(x)\right| \lesssim\left(1-|x|^{2}\right)^{l} \int_{\mathbb{B}}\left|I_{s}^{t} u(y)\right|\left(1-|y|^{2}\right)^{s} \int_{0}^{1} \frac{d \tau}{\{z, y\}^{(1+n+s+l) / 2}} d \nu(y),
$$

where $1+n+s+l \geq 2>0$ and $z$ depends on $\tau$. 
Lemma 6.1 is not applicable for the estimation of the inner integral since $z$ is not a simple multiple of $x$. We resort to [34, Lemma 2.1] which yields

$$
\int_{0}^{1} \frac{d \tau}{\{z, y\}^{(1+n+s+t) / 2}} \lesssim \frac{1}{\{x, y\}^{(n+s+t) / 2}}
$$

Thus

$$
\left(1-|x|^{2}\right)^{l}\left|\partial^{\alpha} A_{i} u(x)\right| \lesssim\left(1-|x|^{2}\right)^{l} \int_{\mathbb{B}}\left|I_{s}^{t} u(y)\right| \frac{1}{\{x, y\}^{(n+s+l) / 2}}\left(1-|y|^{2}\right)^{s} d \nu(y) .
$$

Finally, Theorem 1.6 implies that $\left\|\left(1-|x|^{2}\right)^{l}\left(\partial^{\alpha} A_{i} u\right)(x)\right\|_{L_{q}^{p}} \lesssim\left\|I_{s}^{t} u\right\|_{L_{q}^{p}}$, or equivalently, $\left\|A_{i} u\right\|_{b_{q}^{p}} \lesssim\|u\|_{b_{q}^{p}}$ by Theorem 1.1.

See [7, Theorem 1.2] for $q=0$ and $x^{0}=0$ and [8, Theorem 1.3] for $b_{q}^{p}$ using integer-order derivatives with some conditions on the derivatives of $u$ at $x^{0}$ but on a much larger class of domains.

\section{Review: Spherical Harmonics and Bergman Kernels}

We review the essentials of spherical harmonics, zonal harmonics, and how they give rise to reproducing kernels of harmonic Hardy and Bergman spaces for completeness, because we refer to these facts quite a lot in the rest of the paper. These results are well-known and can be consulted mostly in [1, Chaps. 1, 5, and 8] or [38, Chap. IV].

Lemma 14.1. The dilates of a harmonic function are harmonic.

This allows us to treat a member of $h(\mathbb{B})$ as if it is harmonic on a neighborhood of $\overline{\mathbb{B}}$ in most situations.

The mean value property of harmonic functions written for $\nu_{q}$ takes the form

$$
u(0)=\int_{\mathbb{B}} u d \nu_{q} \quad\left(q>-1, u \in h(\mathbb{B}) \cap L_{q}^{1}\right) .
$$

For $m=0,1,2, \ldots$, let $\mathcal{H}_{m}$ denote the space of all harmonic polynomials homogeneous of degree $m$ with respect to real scalars. By homogeneity, a $u \in \mathcal{H}_{m}$ is determined by its restriction to $\mathbb{S}$, and we freely identify $u$ with its restriction. The restrictions are called spherical harmonics. An important property of spherical harmonics is that if $m \neq l$, then $\mathcal{H}_{m}$ is orthogonal to $\mathcal{H}_{l}$ in $L^{2}(\sigma)$. The spaces $\mathcal{H}_{m}$ are finite-dimensional and

$$
\delta_{m}=\operatorname{dim} \mathcal{H}_{m}=\left(\begin{array}{c}
n+m-2 \\
n-2
\end{array}\right)+\left(\begin{array}{c}
n+m-3 \\
n-2
\end{array}\right) \sim m^{n-2} \quad(m \rightarrow \infty)
$$

by (2.1). Hence the $\mathcal{H}_{m}$ are closed subspaces of $L^{2}(\sigma)$. Moreover,

$$
L^{2}(\sigma)=\bigoplus_{m=0}^{\infty} \mathcal{H}_{m}
$$

which is an orthogonal direct sum. 
Finite-dimensionality of the $\mathcal{H}_{m}$ also tells us that evaluation functionals at points $\eta \in \mathbb{S}$ are bounded on $\mathcal{H}_{m}$, and so $\mathcal{H}_{m}$ is a reproducing kernel Hilbert space. Its reproducing kernel $Z_{m}(\xi, \eta)$ is called the zonal harmonic of degree $m$; thus $Z_{m}$ is a positive definite function. If $\left\{Y_{m 1}, \ldots, Y_{m \delta_{m}}\right\}$ is an orthonormal basis for $\mathcal{H}_{m} \subset L^{2}(\sigma)$, then by general properties of reproducing kernels,

$$
Z_{m}(\xi, \eta)=\sum_{k=1}^{\delta_{m}} Y_{m k}(\xi) \overline{Y_{m k}(\eta)} \quad(\xi, \eta \in \mathbb{S}) .
$$

Particularly, $\mathcal{H}_{0}$ consists of constants, $\delta_{0}=1, Y_{01} \equiv 1$, and $Z_{0} \equiv 1$. Further, $Y_{1 k}(\xi)=\sqrt{n} \xi_{k}$ for $k=1, \ldots, \delta_{1}=n$ and $Z_{1}(\xi, \eta)=n \xi \cdot \eta$.

Zonal harmonics can be extended to positive definite functions on all of $\mathbb{B}$ in one or both variables as $Z_{m}(x, y):=r^{m} \rho^{m} Z_{m}(\xi, \eta)$ by homogeneity. Similarly, we set $Y_{m k}(x):=r^{m} Y_{m k}(\xi)$. These extensions are sometimes called solid spherical harmonics. The reproducing property then takes the form

$$
u(x)=\int_{\mathbb{S}} u(\eta) Z_{m}(x, \eta) d \sigma(\eta)=\left[u(\cdot), Z_{m}(x, \cdot)\right]_{L^{2}(\sigma)} \quad\left(x \in \mathbb{B}, u \in \mathcal{H}_{m}\right) .
$$

By the polar coordinates formula, (14.2) extends to the fact that $\mathcal{H}_{m}$ is orthogonal $\mathcal{H}_{l}$ in $L_{q}^{2}$ too for $q>-1$ if $m \neq l$.

A useful property that results from homogeneity is

$$
Z_{m}(\lambda x, y)=Z_{m}(x, \lambda y) \quad(\lambda \in \mathbb{R}) .
$$

Along the same line, if $L$ is an orthogonal transformation of $\mathbb{R}^{n}$, then

$$
Z_{m}(L x, L y)=Z_{m}(x, y) .
$$

Again by homogeneity, $Z_{m}(0, y)=Z_{m}(x, 0)=0$ for $m>0$. Zonal harmonics are real-valued and symmetric in their variables, that is, $Z_{m}(x, y)=Z_{m}(y, x)$ for $x, y \in \overline{\mathbb{B}}$. Consequently, $Z_{m}$ is harmonic in each of its variables since it lies in $\mathcal{H}_{m}$. We also have

$$
\left|Z_{m}(\xi, \eta)\right| \leq Z_{m}(\xi, \xi)=\delta_{m} \quad(\xi, \eta \in \mathbb{S}) .
$$

We also see that

$$
P(x, \eta)=\sum_{m=0}^{\infty} Z_{m}(x, \eta) \quad(x \in \mathbb{B}, \eta \in \mathbb{S}) .
$$

This relationship in conjunction with (2.5), (6.1), and the recurrence relation in [30, Formula 18.9.7] relates the $Z_{m}$ in the Gegenbauer polynomials via

$$
Z_{m}(\xi, \eta)=\frac{n-2+2 m}{n-2} G_{m}^{n / 2-1}(\xi \cdot \eta) \quad(n>2, m=0,1,2, \ldots) .
$$

The corresponding formulas for $n=2$ are $Z_{m}(\xi, \eta)=m G_{m}^{0}(\xi \cdot \eta)$ for $m>0$ and $Z_{0}=G_{0}^{0}$, which follow from the relation with Chebyshev polynomials and (14.15). 
Theorem 14.1. If $u \in h(\mathbb{B})$, then $u$ has a homogeneous expansion

$$
u(x)=\sum_{m=0}^{\infty} u_{m}(x)
$$

with $u_{m} \in \mathcal{H}_{m}$, the series converging absolutely and uniformly on compact subsets of $\mathbb{B}$. For $|x|<\tau<1$, we have

$$
u_{m}(x)=\frac{1}{\tau^{m}}\left[u(\tau \cdot), Z_{m}(x, \cdot)\right]_{L^{2}(\sigma)}=\frac{1}{\tau^{m}} \int_{\mathbb{S}} u(\tau \eta) Z_{m}(x, \eta) d \sigma(\eta) .
$$

Proof. This is just [1, Corollary 5.34]. The sameness of the value of the integral for all $1>\tau>|x|$ follows from the uniqueness of the homogeneous expansion and is stated in the last sentence of the proof of that result.

Continuing, each $u_{m}$ restricted to $\mathbb{S}$ has itself an expansion in terms of the orthonormal basis $\left\{Y_{m k}\right\}$ of $\mathcal{H}_{m} \subset L^{2}(\sigma)$, and thus

$$
u(x)=\sum_{m=0}^{\infty} r^{m} u_{m}(\xi)=\sum_{m=0}^{\infty} r^{m} \sum_{k=1}^{\delta_{m}} c_{m k} Y_{m k}(\xi)=\sum_{m=0}^{\infty} \sum_{k=1}^{\delta_{m}} c_{m k} Y_{m k}(x)
$$

for $x \in \mathbb{B}$. The convergence is absolute and uniform on compact subsets of $\mathbb{B}$, and

$$
\begin{aligned}
c_{m k} & =\int_{\mathbb{S}} u_{m}(\xi) \overline{Y_{m k}(\xi)} d \sigma(\xi)=\frac{1}{r^{m}} \int_{\mathbb{S}} u_{m}(r \xi) \overline{Y_{m k}(\xi)} d \sigma(\xi) \\
& =\frac{1}{r^{m}} \int_{\mathbb{S}} u(x) \overline{Y_{m k}(\xi)} d \sigma(\xi) \quad(0<r<1)
\end{aligned}
$$

using the orthogonality expressed in (14.2). We also see that this computation of $c_{m k}$ is independent of $r \in(0,1)$. We call the $c_{m k}$ the generalized Fourier coefficients of $u \in h(\mathbb{B})$. This is as close as we get to a Taylor series-like expansion for harmonic functions on $\mathbb{B}$.

The Poisson kernel is also extended to $\mathbb{B}$ in both variables using (14.5) via

$$
\sum_{m=0}^{\infty} Z_{m}(x, y)=\sum_{m=0}^{\infty} Z_{m}\left(\rho x, \frac{y}{\rho}\right)=P(\rho x, \eta)=\frac{1-r^{2} \rho^{2}}{\{x, y\}^{n / 2}}=: P(x, y) \quad(x, y \in \mathbb{B}) .
$$

The reproducing kernels of harmonic weighted Bergman spaces $b_{q}^{2}, q>-1$, on $\mathbb{B}$ have been computed to be

$$
\begin{aligned}
R_{q}(x, y) & =\sum_{m=0}^{\infty} \frac{(1+n / 2+q)_{m}}{(n / 2)_{m}} Z_{m}(x, y) \\
& =: \sum_{m=0}^{\infty} \gamma_{m}(q) Z_{m}(x, y) \quad(q>-1, x, y \in \mathbb{B}),
\end{aligned}
$$

which also defines $\gamma_{m}(q)$; see [29, Proposition 3]. Additionally see [12, Eq. (3.1)] or [4, Eq. (1)] for integer or real $q>-1$; see also [24, p. 25; 26; 1, Eq. (6.21); 41, p. 55] for $q=0$. 
The extended Poisson kernel and the harmonic weighted Bergman kernels converge absolutely on $\mathbb{B} \times \mathbb{B}$, and uniformly if one variable lives in a compact subset of $\mathbb{B}$. The convergence allows us to pass several properties of the $Z_{m}$ to $P$ and $R_{q}$. Therefore $P$ and the $R_{q}$ are symmetric in their variables and harmonic as a function of each.

The reproducing property of the Bergman kernels is

$$
u(x)=\int_{\mathbb{B}} u(y) R_{q}(x, y) d \nu_{q}(y) \quad\left(u \in b_{q}^{2}, q>-1\right),
$$

and such a form without derivatives is standard for weighted Bergman spaces. As is common, this equality extends to the larger space $b_{q}^{1}$.

Proposition 14.1. Let $q>-1$. Then (14.13) holds for all $u \in b_{q}^{1}$ and hence for all $u \in b_{q}^{p}$.

See [14, Lemma 2.11] for a proof. An early version for $u \in h^{\infty}$ with $q$ a nonnegative integer is in [12, Proposition 3.1].

The computation yielding $R_{q}$ is valid only for $q>-1$, but $R_{-1}$ also perfectly makes sense and is none other than the Poisson kernel $P$ since $\gamma_{m}(-1)=1$ for all $m$. The space that $R_{-1}=P$ reproduces is called the harmonic Hardy space $h^{2}$. Its members $u$ have boundary values in $L^{2}(\sigma)$, and thus

$$
\|u\|_{b_{-1}^{2}}^{2}=\|u\|_{h^{2}}^{2}=\int_{\mathbb{S}}|u|^{2} d \sigma
$$

and

$$
u(x)=\int_{\mathbb{S}} u(\eta) P(x, \eta) d \sigma(\eta) \quad\left(u \in h^{2}, x \in \mathbb{B}\right)
$$

by $(2.2)$.

It is instructive to have a look at the simplest case $n=2$. The homogeneous expansion of a $u \in h(\mathbb{D})$ with suitable boundary behavior is its Fourier series expansion on the unit circle. Then $\delta_{m}=2$ and an orthonormal basis for $\mathcal{H}_{m}$ is $\left\{Y_{m 1}(\xi)=e^{i m \theta}, Y_{m 2}(\xi)=e^{-i m \theta}: \xi=e^{i \theta}\right\}$ for $m>0$. Letting also $\eta=e^{i \phi}$, we can write

$$
Z_{m}(x, y)=x^{m} \bar{y}^{m}+\bar{x}^{m} y^{m}=2 r^{m} \rho^{m} \cos m(\theta-\phi) \quad(m=1,2, \ldots)
$$

since now $x \cdot y=2 r \rho \operatorname{Re}(\xi \bar{\eta})$. Then also

$$
\begin{aligned}
P\left(x, e^{i(\theta-\phi)}\right) & =1+2 \sum_{m=1}^{\infty} r^{m} \cos m(\theta-\phi) \\
& =\sum_{m=-\infty}^{\infty} r^{|m|} e^{i m(\theta-\phi)} \\
& =\sum_{m=-\infty}^{\infty} r^{|m|}(\xi \bar{\eta})^{m}
\end{aligned}
$$


in its more familiar form. This gives us the familiar forms $Y_{m}(x)=x^{m}=r^{m} e^{i m \theta}$ for $m>0$ and $Y_{m}(x)=r^{|m|} e^{i m \theta}$ for $m<0$ of the basis elements.

More interesting is that $\gamma_{m}(q)=(2+q)_{m} / m$ ! for $q>-2$ and thus

$$
\begin{aligned}
R_{q}(x, y) & =1+\sum_{m=1}^{\infty} \frac{(2+q)_{m}}{m !}\left(x^{m} \bar{y}^{m}+\bar{x}^{m} y^{m}\right) \\
& =\frac{1}{(1-x \bar{y})^{2+q}}+\frac{1}{(1-\bar{x} y)^{2+q}}-1=2 \operatorname{Re} K_{q}(x, y)-1,
\end{aligned}
$$

where the $K_{q}(x, y)$ are the holomorphic weighted Bergman kernels; see [42, p. 357]. Similarly, the $K_{q}$ make sense for $q>-2$ even though weighted Bergman spaces are defined only for $q>-1$.

\section{Acknowledgments}

This research is partially supported by TÜBITAK under Research Project Grant $108 \mathrm{~T} 329$.

\section{References}

[1] S. Axler, P. Bourdon and W. Ramey, Harmonic Function Theory, 2nd edn., Graduate Texts in Mathematics, Vol. 137 (Springer, New York, 2001).

[2] F. Beatrous and J. Burbea, Holomorphic Sobolev spaces on the ball, Dissertationes Math. 276 (1989) 57 pp.

[3] J. Bergh and J. Löfström, Interpolation Spaces: An Introduction, Grundlehren der Mathematischen Wissenschaften, Vol. 223 (Springer, Berlin, 1976).

[4] O. Blasco and S. Pérez-Esteva, $L^{p}$ continuity of projectors of weighted harmonic Bergman spaces, Collect. Math. 51 (2000) 49-58.

[5] B. R. Choe, H. Koo and K. Na, Positive Toeplitz operators of Schatten-Herz type, Nagoya Math. J. 185 (2007) 31-62.

[6] B. R. Choe, H. Koo and K. Nam, Optimal norm estimate of operators related to the harmonic Bergman projection on the ball, Tohoku Math. J. 62 (2010) 357-374.

[7] B. R. Choe, H. Koo and H. Yi, Derivatives of harmonic Bergman and Bloch functions on the ball, J. Math. Anal. Appl. 260 (2001) 100-123.

[8] B. R. Choe, H. Koo and H. Yi, Projections for harmonic Bergman spaces and applications, J. Funct. Anal. 216 (2004) 388-421.

[9] B. R. Choe and H. Y. J. Lee, Note on atomic decompositions of harmonic Bergman functions, in Complex Analysis and Its Applications, OCAMI Studies, Vol. 2 (Osaka Municipal University, Osaka, 2007), pp. 11-24.

[10] B. R. Choe, Y. J. Lee and K. Na, Toeplitz operators on harmonic Bergman spaces, Nagoya Math. J. 174 (2004) 165-186.

[11] E. S. Choi and K. Na, Characterizations of the harmonic Bergman space on the ball, J. Math. Anal. Appl. 353 (2009) 375-385.

[12] R. R. Coifman and R. Rochberg, Representation theorems for holomorphic and harmonic functions in $L^{p}$, Astérisque $\mathbf{7 7}$ (1980) 12-66.

[13] S. Gergün, H. T. Kaptanoğlu and A. E. Üreyen, Reproducing kernels for harmonic Besov spaces on the ball, C. R. Math. Acad. Sci. Paris 347 (2009) 735-738.

[14] M. Jevtić and M. Pavlović, Harmonic Bergman functions on the unit ball in $\mathbb{R}^{n}$, Acta Math. Hungar. 85 (1999) 81-96. 
[15] M. Jevtić and M. Pavlović, Harmonic Besov spaces on the unit ball in $\mathbb{R}^{n}$, Rocky Mountain J. Math. 31 (2001) 1305-1316.

[16] H. Kang and H. Koo, Estimates of the harmonic Bergman kernel on smooth domains, J. Funct. Anal. 185 (2001) 220-239.

[17] H. T. Kaptanoğlu, Bergman projections on Besov spaces on balls, Illinois J. Math. 49 (2005) 385-403.

[18] H. T. Kaptanoğlu, Reproducing kernels and radial differential operators for holomorphic and harmonic Besov spaces on unit balls: A unified view, Comput. Methods Funct. Theory 10 (2010) 483-500.

[19] H. T. Kaptanoğlu and A. E. Üreyen, Analytic properties of Besov spaces via Bergman projections, Contemp. Math. 455 (2008) 169-182.

[20] D. Karp, private communication (2010).

[21] H. Koo, K. Nam and H. Yi, Weighted harmonic Bergman functions on half-spaces, J. Korean Math. Soc. 42 (2005) 975-1002.

[22] E. Ligocka, The Sobolev spaces of harmonic functions, Studia Math. 84 (1986) 79-87.

[23] E. Ligocka, Estimates in Sobolev norms $\|\cdot\|_{p}^{s}$ for harmonic and holomorphic functions and interpolation between Sobolev and Hölder spaces of harmonic functions, Studia Math. 86 (1987) 255-271.

[24] E. Ligocka, On the reproducing kernel for harmonic functions and the space of Bloch harmonic functions on the unit ball in $\mathbb{R}^{n}$, Studia Math. 87 (1987) 23-32.

[25] E. Ligocka, On the space of Bloch harmonic functions and interpolation of spaces of harmonic and holomorphic functions, Studia Math. 87 (1987) 223-238.

[26] E. Ligocka, Corrigendum to the paper "On the reproducing kernel for harmonic functions and the space of Bloch harmonic functions on the unit ball in $\mathbb{R}^{n}$ ", Studia Math. 101 (1992) 319.

[27] C. W. Liu and J. H. Shi, Invariant mean-value property and $\mathcal{M}$-harmonicity in the unit ball of $\mathbb{R}^{n}$, Acta Math. Sin. 19 (2003) 187-200.

[28] C. Liu, J. Shi and G. Ren, Duality for harmonic mixed-norm spaces in the unit ball of $\mathbb{R}^{n}$, Ann. Sci. Math. Québec 25 (2001) 179-197.

[29] J. Miao, Reproducing kernels for harmonic Bergman spaces of the unit ball, Monatsh. Math. 125 (1998) 25-35.

[30] F. W. J. Olver, D. W. Lozier, R. F. Boisvert and C. W. Clark (eds.), NIST Handbook of Mathematical Functions (Cambridge University, New York, 2010).

[31] R. Otáhalová, Weighted reproducing kernels and Toeplitz operators on harmonic Bergman spaces on the real ball, Proc. Amer. Math. Soc. 136 (2008) 2483-2492.

[32] S. Pérez-Esteva, Duality on vector-valued weighted harmonic Bergman spaces, Studia Math. 118 (1996) 37-47.

[33] G. Ren, Harmonic Bergman spaces with small exponents in the unit ball, Collect. Math. 53 (2003) 83-98.

[34] G. Ren and U. Kähler, Weighted harmonic Bloch spaces and Gleason's problem, Complex Var. Theory Appl. 48 (2003) 235-245.

[35] G. Ren and U. Kähler, Weighted Lipschitz continuity and harmonic Bloch and Besov spaces in the unit real ball, Proc. Edinb. Math. Soc. 48 (2005) 743-755.

[36] W. Rudin, Function Theory in the Unit Ball of $\mathbb{C}^{n}$, Grundlehren der Mathematischen Wissenschaften, Vol. 241 (Springer, New York, 1980).

[37] A. L. Shields and D. L. Williams, Bounded projections, duality, and multipliers in spaces of analytic functions, Trans. Amer. Math. Soc. 162 (1971) 287-302.

[38] E. M. Stein and G. Weiss, Introduction to Fourier Analysis on Euclidean Spaces, Princeton Mathematical Series, Vol. 32 (Princeton University, Princeton, 1971).

[39] S. Stević, On harmonic function spaces, J. Math. Soc. Japan 57 (2005) 781-802. 
[40] M. Stoll, Harmonic function theory on real hyperbolic space, unpublished manuscript (2000).

[41] K. Stroethoff, Harmonic Bergman spaces, in Holomorphic Spaces, Mathematical Sciences Research Institute Publications, Vol. 33 (Cambridge University, Cambridge, 1998), pp. 51-63.

[42] Z. Wu, Operators on harmonic Bergman spaces, Integral Equations Operator Theory 24 (1996) 352-371.

[43] R. Yoneda, A characterization of the harmonic Bloch space and the harmonic Besov spaces by an oscillation, Proc. Edinb. Math. Soc. 45 (2002) 229-239.

[44] K. Zhu, Spaces of Holomorphic Functions in the Unit Ball, Graduate Texts in Mathematics, Vol. 226 (Springer, New York, 2005). 